\title{
Status of Electroweak Tests with Heavy Quarks
}

\author{
Klaus Mönig \\ CERN/EP ${ }^{a}$
}

\begin{abstract}
The measurements of the partial decay-widths and forward-backward asymmetries for $\mathrm{Z} \rightarrow \mathrm{b} \overline{\mathrm{b}}$ and $\mathrm{Z} \rightarrow \mathrm{c} \overline{\mathrm{c}}$ test the $\mathrm{Z}$ couplings to the initial state $\mathrm{e}^{+} \mathrm{e}^{-}$pair and the heavy quarks in the final state.

The four LEP detectors have registered about four million hadronic $\mathrm{Z}$ decays each and SLD at SLC has recorded $300000 \mathrm{Z}$ decays with highly polarised electron beams. The high statistics as well as the good tracking, vertexing and particle identification capabilities of the detectors allow high precision measurements of these quantities.

The measurements of the electroweak observables with heavy quarks are reviewed. The results of the different analyses are combined and interpreted within the framework of the Standard Model of electroweak interactions. In all cases good agreement with the Standard Model predictions is found, severely limiting the room for modifications of these quantities from new physics.
\end{abstract}

(Submitted to Reports on Progress in Physics) 


\section{Introduction}

One of the main goals of the $\mathrm{e}^{+} \mathrm{e}^{-}$colliders LEP at CERN and SLC at SLAC is the test of the Glashow Salam Weinberg Model of electroweak interactions [1], which will be referred to as the Standard Model (SM) in this paper. In the past this model has described all effects successfully, however the precision of earlier experiments was such that Born level calculations were sufficient. LEP and SLC measure for the first time electroweak observables with an accuracy that makes loop corrections necessary, so that the Standard Model is tested as a quantum theory.

From 1989 to 1995 LEP has run at centre-of-mass energies close to the mass of the Z boson ${ }^{1}$. About four million Z-decays have been observed by each of the four experiments. The beam energy can be measured to a precision of a few $\mathrm{MeV}$ as a consequence of the circular geometry of the accelerator. The LEP experiments have thus been able to measure the mass and width of the Z, its branching ratios, and various asymmetries, that are sensitive to the weak mixing angle $\sin ^{2} \theta_{W}$ with good accuracy. Since 1996 LEP is running at higher energies, above the threshold for $\mathrm{W}$ pair production. Apart from a precision measurement of the $\mathrm{W}$ mass, the main emphasis of this run is a Born level test of the vector boson self-couplings and the search for new particles, especially the Higgs boson. First results at these energies are available [2,3], however, they will not be included in this review.

SLC, running also close to the $\mathrm{Z}$ pole, has delivered about 300000 Z-decays until the end of 1997 to its one experiment, SLD. Using the linear collider technology it is possible to polarise the electron beam to up to $80 \%$. This allows a clean separation of initial state and final state couplings and consequently for very competitive measurements of $\sin ^{2} \theta_{W}$.

Electroweak results at LEP with particular emphasis on the Z lineshape and leptonic observables have been recently reviewed in [4]. At LEP and SLD, b- and c-quarks can be tagged with high efficiency and purity so that additional observables with identified c- and especially b-quark final states are accessible. The b-quark is the isospin partner of the heavy top quark. This makes effects visible in the $Z \mathrm{~b} \bar{b}$ vertex which are not present in other observables. This vertex can be tested by measuring the partial decay width of the $Z$ decaying into $b \bar{b}$. On the contrary, in the forward backward asymmetry for b-quarks at LEP the sensitivity to the final state couplings is heavily suppressed, so that it turns out to be one of the most sensitive tests of the $\mathrm{Z}$ couplings to leptons.

This article will review the LEP and SLD precision tests of the Standard Model using heavy flavour observables. After a general introduction and definition of the observables the measurements will be described in detail and the results will be interpreted within the Standard Model of electroweak interactions.

\section{Electroweak Observables with Heavy Quarks}

\subsection{Born level formulae}

In $\mathrm{e}^{+} \mathrm{e}^{-}$-annihilation, generally four quantities can be measured for any given fermion, namely the total cross section, the forward backward asymmetry, the final state polarisation and its forward-backward asymmetry. The two latter are not accessible for quarks since strong decays conserve parity and weak decays in general occur from scalar meson states, therefore destroying the polarisation information. If initial state beam polarisation is available, addi-

\footnotetext{
${ }^{1}$ Throughout this paper the convention $\hbar=\mathrm{c}=1$ will be used.
} 
tional information can be obtained by measuring these quantities separately for the different beam polarisations.

The measurements discussed here are all performed close to the top of the Z-resonance where the Z-exchange (see figure 1) contributes more than a factor 100 more to the total cross section than photon exchange. In addition, exactly at the Z-mass, the Z-exchange amplitude is purely imaginary, so that the $\gamma-\mathrm{Z}$ interference term vanishes. For these reasons, processes will in the following be treated as being pure Z-exchange where small corrections for $\gamma$-exchange and $\gamma-\mathrm{Z}$ interference have to be applied.

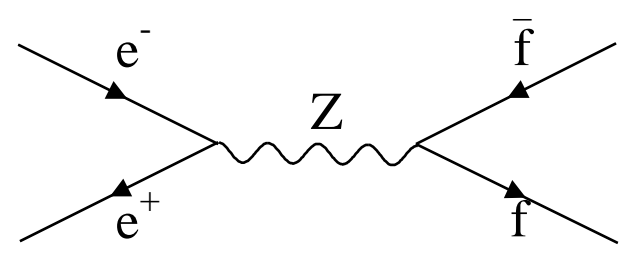

Figure 1: Born level Feynman diagram of the dominant LEP/SLC process $\mathrm{e}^{+} \mathrm{e}^{-} \rightarrow \mathrm{Z} \rightarrow \mathrm{f} \overline{\mathrm{f}}$.

Then, in Born approximation, the differential cross section $\mathrm{e}^{+} \mathrm{e}^{-} \rightarrow \mathrm{f} \overline{\mathrm{f}}(\mathrm{f} \neq \mathrm{e})$ for massless fermions and unpolarised beams at a centre of mass energy $\sqrt{s}$ is given by [5]

$$
\frac{d \sigma_{\mathrm{f}}(s)}{d \cos \theta}=\frac{N_{C}^{\mathrm{f}} G_{\mathrm{F}}^{2} m_{\mathrm{Z}}^{4}}{16 \pi} \frac{s}{\left(s-m_{\mathrm{Z}}^{2}\right)^{2}+\frac{s^{2}}{m_{\mathrm{Z}}^{2}} \Gamma_{\mathrm{Z}}^{2}}\left[\left(v_{\mathrm{e}}^{2}+a_{\mathrm{e}}^{2}\right)\left(v_{\mathrm{f}}^{2}+a_{\mathrm{f}}^{2}\right)\left(1+\cos ^{2} \theta\right)+2 v_{\mathrm{e}} a_{\mathrm{e}} v_{\mathrm{f}} a_{\mathrm{f}} \cos \theta\right],
$$

where $m_{\mathrm{Z}}$ and $\Gamma_{\mathrm{Z}}$ denote the mass and width of the $\mathrm{Z}$ respectively, $G_{\mathrm{F}}$ the Fermi coupling constant measured in $\mu$-decays [6] and $\cos \theta$ the polar angle of the fermion $\mathrm{f}$ with respect to the incoming electron direction. The colour factor $N_{C}^{\mathrm{f}}$ is 1 for leptons and 3 for quarks. $a_{\mathrm{f}}$ and $v_{\mathrm{f}}$ are the axial vector and vector coupling of the fermion $\mathrm{f}$ to the $\mathrm{Z}$. They are given by $a_{\mathrm{f}}=i_{3}^{(\mathrm{f})}$ and $v_{\mathrm{f}}=a_{\mathrm{f}} \cdot\left(1-4\left|q_{\mathrm{f}}\right| \sin ^{2} \theta_{W}\right)$, where $i_{3}^{(\mathrm{f})}$ is the third component of the weak isospin and $q_{\mathrm{f}}$ the charge of the fermion f. $\theta_{W}$ is the weak mixing angle which is, in its on-shell definition [7], given by $\sin ^{2} \theta_{W}=1-\frac{m_{\mathrm{W}}^{2}}{m_{\mathrm{Z}}^{2}}$.

The partial width of the $\mathrm{Z}$ decaying into $\mathrm{f} \overline{\mathrm{f}}$ is given by

$$
\Gamma_{\mathrm{f}}=\frac{N_{C}^{\mathrm{f}} G_{\mathrm{F}} m_{\mathrm{Z}}^{3}}{6 \pi \sqrt{2}}\left(v_{\mathrm{f}}^{2}+a_{\mathrm{f}}^{2}\right)
$$

so that the total cross section can be written as

$$
\sigma_{\mathrm{f}}(s)=\frac{12 \pi \Gamma_{\mathrm{e}} \Gamma_{\mathrm{f}}}{m_{\mathrm{Z}}^{2}} \frac{s}{\left(s-m_{\mathrm{Z}}^{2}\right)^{2}+\frac{s^{2}}{m_{\mathrm{Z}}^{2}} \Gamma_{\mathrm{Z}}^{2}} .
$$

The forward backward asymmetry,

$$
A_{\mathrm{FB}}^{\mathrm{f}}=\frac{\sigma(\cos \theta>0)-\sigma(\cos \theta<0)}{\sigma_{\mathrm{tot}}}
$$

where $\sigma_{\text {tot }}$ is the total cross section, is given by $A_{\mathrm{FB}}^{\mathrm{f}}=\frac{3}{4} \mathcal{A}_{\mathrm{e}} \mathcal{A}_{\mathrm{f}}, \mathcal{A}_{\mathrm{f}}=\frac{2 v_{\mathrm{f}} a_{\mathrm{f}}}{v_{\mathrm{f}}^{2}+a_{\mathrm{f}}^{2}}$. The angular dependence of the cross section can be expressed as

$$
\frac{d \sigma}{d \cos \theta}=\sigma_{\text {tot }}\left(\frac{3}{8}\left(1+\cos ^{2} \theta\right)+A_{\mathrm{FB}} \cos \theta\right) .
$$


If polarised beams are available with electron polarisation $\pm \mathcal{P}$, the cross section asymmetry $A_{\mathrm{LR}}=\frac{1}{\mathcal{P}} \frac{\sigma_{L}-\sigma_{R}}{\sigma_{L}+\sigma_{R}}$ can also be measured where $\sigma_{L / R}$ denotes the total cross section for left $/$ righthanded electrons. $A_{\mathrm{LR}}$ is given by $A_{\mathrm{LR}}=\mathcal{A}_{\mathrm{e}}$, independent of the final state.

The forward-backward asymmetry for polarised beams is given by

$$
A_{\mathrm{FB}}^{\mathrm{f}}=\frac{3}{4} \mathcal{A}_{\mathrm{f}} \frac{\mathcal{A}_{\mathrm{e}}-\mathcal{P}}{1-\mathcal{A}_{\mathrm{e}} \mathcal{P}},
$$

so that the left-right-forward-backward asymmetry

$$
A_{\mathrm{LR}, \mathrm{FB}}^{\mathrm{f}}=\frac{1}{\mathcal{P}} \frac{\left[\sigma_{L}^{\mathrm{f}}(\cos \theta>0)-\sigma_{L}^{\mathrm{f}}(\cos \theta<0)\right]-\left[\sigma_{R}^{\mathrm{f}}(\cos \theta>0)+\sigma_{R}^{\mathrm{f}}(\cos \theta<0)\right]}{\sigma_{L}^{\mathrm{f}}+\sigma_{R}^{\mathrm{f}}}
$$

can be constructed which is sensitive only to the final state coupling $A_{\mathrm{LR}, \mathrm{FB}}^{\mathrm{f}}=\frac{3}{4} \mathcal{A}_{\mathrm{f}}$.

\subsection{Radiative corrections}

Apart from corrections for photon exchange and $\gamma-\mathrm{Z}$ interference, three different types of radiative corrections have to be applied to the Born-level formulae described above:

- photonic corrections;

- final state QCD corrections;

- genuine electroweak corrections.

Photonic corrections are obtained attaching photons at any possible place on the Born-level diagrams, but by far the largest part is given by initial state radiation (ISR). These corrections can be calculated reliably in QED, by folding a radiator function with the cross section at the centre of mass energy after photon radiation, and they will not be discussed here any further. It should be noted that at centre of mass energies close to the $\mathrm{Z}$ resonance the bulk of the radiation is soft, so that the kinematics of the events are typically only minimally distorted.

Final state QCD corrections are, at first order, given by gluons attached to the outgoing quark lines. Their contributions to the total cross section are known to third order in the strong coupling constant $\alpha_{s}$ [8], and their main effect is to put an additional uncertainty on the interpretation of $\Gamma_{Z}$ from the uncertainty in $\alpha_{s}$. Hard gluon radiation, however, seriously distorts the kinematics of the events, influencing the hadronic observables in a more subtle way. These influences can be calculated using exact second order QCD matrix elements or parton shower Monte Carlo programs that also include hadronisation, like JETSET [9] or HERWIG [10].

As an example, the forward backward asymmetry for massless quarks receives in first order a correction factor $\left(1-\frac{\alpha_{s}}{\pi}\right)$ due to hard gluon radiation, if, as in the definition, the direction of the quark is used. If however, as is closer to the experimental analyses, the direction of the thrust axis is used, the correction reduces to $\left(1-0.893 \frac{\alpha_{s}}{\pi}\right)$ [11]. In addition, the shape of the angular distribution is modified and the size of the correction depends on the experimental cuts. More details on this correction will be discussed in section 5.1.5.

The really interesting radiative corrections are the genuine electroweak corrections. Particles being too heavy to be produced directly can still appear in loop diagrams so that their effect may be visible in precision measurements of electroweak quantities. Close to the Z-resonance, all diagrams that involve non-resonant massive particles are negligible, so that the only relevant 
corrections are those to the Z-propagator and the Zff $\bar{f}$ vertex. Examples of such corrections are shown in figure 2. Due to this feature the electroweak radiative corrections can be parametrised to very good approximation by effective coupling constants, replacing $a_{\mathrm{f}}$ and $v_{\mathrm{f}}$ in the Born-level formulae by $g_{A f}=\sqrt{1+\Delta \rho_{\mathrm{f}}} a_{\mathrm{f}}$ and by $g_{V \mathrm{f}}=g_{A \mathrm{f}}\left(1-4\left|q_{\mathrm{f}}\right| \sin ^{2} \theta_{\mathrm{eff}}^{\mathrm{f}}\right)[5,12]$.

a)

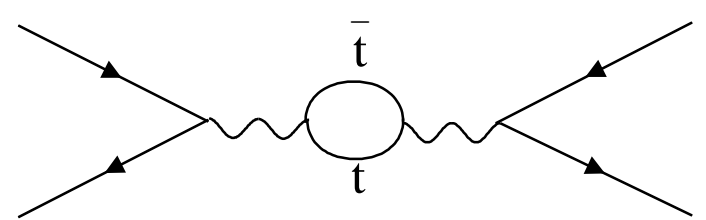

b)

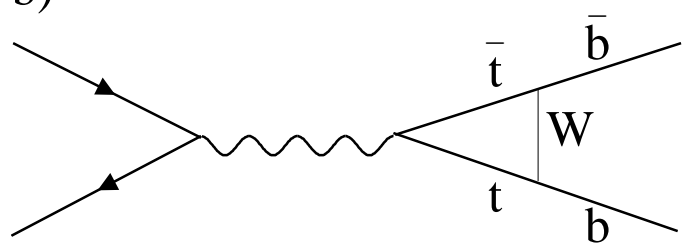

Figure 2: Examples of genuine electroweak corrections to the Z-propagator (a) and the Z $\mathrm{b} \overline{\mathrm{b}}$ vertex (b).

If one assumes lepton universality, all leptonic observables at LEP and SLD can thus be described by two parameters, $\Delta \rho_{\ell}$ and $\sin ^{2} \theta_{\text {eff }}^{\ell}$. In the quark sector, the b-quark has to be separated from the others since it is the partner of the very heavy top quark, and thus receives additional corrections like the one shown in figure 2 b) [13,14]. Also, Higgs-like hypothetical new particles couple proportionally to the fermion mass, breaking universality. For example, in supersymmetric models graphs involving charged Higgses or charginos give a sizeable contribution to the $\mathrm{Zb} \bar{b}$ vertex.

Table 1 shows $\Delta \rho_{\mathrm{f}}$ and $\sin ^{2} \theta_{\text {eff }}^{\mathrm{f}}$ predicted by the Standard Model for different top and Higgs masses. It can be seen that the differences between the different flavours remain constant apart from the b, so that $\Delta \rho_{\ell}$ and $\sin ^{2} \theta_{\text {eff }}^{\ell}$ describe all observables not involving b-quarks.

\begin{tabular}{|c|c|c|c|}
\hline & $\begin{array}{c}m_{\mathrm{t}}=175 \mathrm{GeV} \\
m_{\mathrm{H}}=300 \mathrm{GeV}\end{array}$ & $\pm 6 \mathrm{GeV}$ & \\
\hline$\Delta \rho_{\ell}$ & 0.00464 & \pm 0.00054 & ${ }_{-230}^{+700} \mathrm{GeV}$ \\
\hline$\Delta \rho_{U}-\Delta \rho_{\ell}$ & 0.0006926 & +0.00082 \\
$\Delta \rho_{D}-\Delta \rho_{\ell}$ & 0.00166 & 0 & 0 \\
$\Delta \rho_{b}-\Delta \rho_{\ell}$ & -0.01117 & $\mp$ & 0 \\
\hline $\sin ^{2} \theta_{\text {eff }}^{\ell}$ & 0.23221 & $\mp 0.00100$ & -0.00010 \\
$\sin ^{2} \theta_{\text {eff }}^{U}-\sin ^{2} \theta_{\text {eff }}^{\ell}$ & -0.00011 & 0 & -0.00008 \\
$\sin ^{2} \theta_{\text {eff }}^{D}-\sin ^{2} \theta_{\text {eff }}^{\ell}$ & -0.00023 & 0 & 0 \\
$\sin ^{2} \theta_{\text {eff }}^{\mathrm{b}}-\sin ^{2} \theta_{\text {eff }}^{\ell}$ & 0.00133 & \pm 0.00011 & 0 \\
\hline
\end{tabular}

Table 1: $\Delta \rho$ and $\sin ^{2} \theta_{\text {eff }}$ as predicted by the Standard Model for different top and Higgs masses $(\ell=\mathrm{e}, \mu, \tau, U=\mathrm{u}, \mathrm{c}, D=\mathrm{d}, \mathrm{s})$.

A significant contribution to $\sin ^{2} \theta_{\text {eff }}$ is coming from light fermion loops which introduce a running of the fine structure constant $\alpha_{Q E D}$. Due to the largeness of $\alpha_{s}$ at low energy the contribution from quark loops cannot be calculated reliably in perturbation theory but has to be taken from the $\mathrm{e}^{+} \mathrm{e}^{-}$hadronic cross section using the optical theorem. In this report a value of $\alpha_{Q E D}\left(m_{\mathrm{Z}}^{2}\right)=1 /(128.896 \pm 0.090)$ [15] is used, where the assumed error introduces an uncertainty of \pm 0.00023 in the prediction of $\sin ^{2} \theta_{\text {eff }}$. Analyses of $\alpha_{Q E D}\left(m_{\mathrm{Z}}^{2}\right)$ have recently become available that use perturbative QCD for energies above $1.7 \mathrm{GeV}$, thus reducing the error by about a factor of four $[16,17]$. However, since the validity of these analyses is still under discussion, the more conservative result will be used in this report. 


\subsection{Experimentally measured observables}

The Z-mass and width and its partial decay widths into leptons and hadrons $\left(\Gamma_{\text {had }}=\sum_{\text {quarks }} \Gamma_{\mathrm{q}}\right)$ are measured from an energy scan around the Z-pole [4]. The full information on the $Q \bar{Q}(Q=$ b, c) cross section can thus be retained if the ratio of the $Q \bar{Q}$ to the total hadronic cross section is measured. For pure Z-exchange this ratio is equal to the ratio of partial widths ${ }^{2} R_{Q}^{0}=\frac{\Gamma_{Q}}{\Gamma_{\text {bad }}}$.

Experimentally this ratio is favoured since it is statistically and systematically uncorrelated with the Z-scan observables. Theoretically it has the advantage that in the ratio most of the QED-, QCD- and Z-propagator corrections cancel, so that only corrections to the $Z Q \bar{Q}$ vertex remain which cannot be tested by other means. The total corrections needed to go from the cross section ratios $R_{\mathrm{b}}$ and $R_{\mathrm{c}}$ to the ratios of partial Z-widths $R_{\mathrm{b}}^{0}$ and $R_{\mathrm{c}}^{0}$ are

$$
\begin{aligned}
& R_{\mathrm{b}}^{0}=R_{\mathrm{b}}+0.0002, \\
& R_{\mathrm{c}}^{0}=R_{\mathrm{c}}-0.0003,
\end{aligned}
$$

mainly due to photon exchange.

Inside the Standard Model, $R_{\mathrm{c}}$ is predicted with very small uncertainties, so that its measurement directly tests the model. The predicted $R_{\mathrm{b}}$ depends on the value of the top quark mass, but for a top mass error of $5 \mathrm{GeV}$ the error on the prediction is less than a quarter of the current experimental error. Outside the Standard Model, $R_{\mathrm{b}}$ is especially interesting, as explained in section 2.2, and many calculations of new physics effects on $R_{\mathrm{b}}$ are available [18].

Contrary to $R_{\mathrm{b}}$ and $R_{\mathrm{c}}$, the forward-backward asymmetries at LEP are mainly sensitive to the electron couplings $\mathcal{A}_{\mathrm{e}}$. As can be seen from table $1, \sin ^{2} \theta_{\text {eff }}^{\ell}-\sin ^{2} \theta_{\text {eff }}^{\mathrm{c}}$ is constant and small within the Standard Model, so that $A_{\mathrm{FB}}^{\mathrm{c}}$ measures $\sin ^{2} \theta_{\mathrm{eff}}^{\ell}$. On the contrary $\sin ^{2} \theta_{\mathrm{eff}}^{\ell}-\sin ^{2} \theta_{\mathrm{eff}}^{\mathrm{b}}$ varies by about the same amount as $\sin ^{2} \theta_{\text {eff }}^{\ell}$ itself. However, because the b-charge is only one third and $\frac{d \mathcal{A}_{\mathrm{b}}}{d\left(g_{V \mathrm{~b}} / g_{A \mathrm{~b}}\right)}=0$ for $g_{V \mathrm{~b}} / g_{A \mathrm{~b}}=1$, the sensitivity of $A_{\mathrm{FB}}^{\mathrm{b}}$ to $\sin ^{2} \theta_{\text {eff }}^{\mathrm{b}}$ is suppressed by a factor 50 with respect to $\sin ^{2} \theta_{\mathrm{eff}}^{\ell}$, so that also $A_{\mathrm{FB}}^{\mathrm{b}}$ is a clean measure of $\sin ^{2} \theta_{\mathrm{eff}}^{\ell}$ if new physics appears only in loop diagrams.

The $\gamma$-Z-interference contribution to the forward backward asymmetries depends on the axial vector couplings of the $Z$, which is, for the initial state electron, much larger than the vector coupling. Thus $A_{\mathrm{FB}}$ is modified significantly if not measured exactly at the Z-pole [19]. For the same reason, ISR corrections, which correspond to an effective shift of $\sqrt{s}$, are also sizable. The corrections needed to go from the asymmetries measured at $\sqrt{s}=91.26 \mathrm{GeV}$ to the pole asymmetries $A_{\mathrm{FB}}^{0, \mathrm{~b}}$ and $A_{\mathrm{FB}}^{0, \mathrm{c}}$ are summarised in table 2 . It should be noted that the axial couplings vary much less than the vector couplings: e.g. if the top-quark mass is varied between 175 and $220 \mathrm{GeV}$, which changes $A_{\mathrm{FB}}^{\mathrm{b}}$ and $A_{\mathrm{FB}}^{\mathrm{c}}$ by about $10 \%$, all numbers in table 2 stay constant within the precision quoted.

The left-right-forward-backward asymmetries measured by SLD are predicted essentially without uncertainty by the Standard Model, and contain no additional information if new physics is only present in loop diagrams. However, if new physics exists on the Born level, e.g. like a heavy $\mathrm{Z}^{\prime}$, it can be uniquely identified in $\mathcal{A}_{\mathrm{b}}$ and $\mathcal{A}_{\mathrm{c}}$ without uncertainties due to unknown Standard Model parameters [20].

All quantities of interest including the full radiative corrections can be calculated using analytical programs. For the results presented in this report the program ZFITTER [21] is used. However several other programs exist that have been found to produce nearly identical results $[22,23]$. The Standard Model predictions, corrected to pure Z-exchange, are summarised in table 3.

\footnotetext{
${ }^{2}$ The ${ }^{0}$ always indicates that a quantity is corrected to pure Z-exchange.
} 


\begin{tabular}{|l|l|l|}
\hline Source & $\delta A_{\mathrm{FB}}^{\mathrm{b}}$ & $\delta A_{\mathrm{FB}}^{\mathrm{c}}$ \\
\hline$\sqrt{s}=m_{\mathrm{Z}}$ & -0.0013 & -0.0034 \\
QED corrections & +0.0041 & +0.0104 \\
$\gamma, \gamma-\mathrm{Z}$ & -0.0003 & -0.0008 \\
\hline Total & +0.0025 & +0.0062 \\
\hline
\end{tabular}

Table 2: Corrections to be applied to the quark asymmetries. The corrections are to be understood as $A_{\mathrm{FB}}^{0}=A_{\mathrm{FB}}(91.26 \mathrm{GeV})+\sum_{i}\left(\delta A_{\mathrm{FB}}\right)_{i}$. The term labelled " $\gamma, \gamma-\mathrm{Z}$ " also contains small corrections from mass effects.

\begin{tabular}{|c|c|c|c|c|}
\hline & $\begin{array}{c}m_{\mathrm{t}}=175 \mathrm{GeV} \\
m_{\mathrm{H}}=300 \mathrm{GeV} \\
\end{array}$ & $\pm 6 \mathrm{GeV}$ & & \\
& $\alpha_{Q E D}\left(m_{\mathrm{Z}}^{2}\right)^{-1}=128.896$ & & ${ }_{-230} \mathrm{GeV}$ & \\
\hline$A_{\mathrm{FB}}^{0, \mathrm{~b}}$ & 0.0998 & \pm 0.0010 & ${ }_{-0.0037}^{+0.0042}$ & $\mp 0.0013$ \\
$A_{\mathrm{FB}}^{0, \mathrm{c}}$ & 0.0711 & \pm 0.0008 & ${ }_{-0.0028}$ & $\mp 0.0010$ \\
$R_{\mathrm{b}}^{0}$ & 0.2158 & $\mp 0.0002$ & \pm 0 & \pm 0 \\
$R_{\mathrm{c}}^{0}$ & 0.1722 & \pm 0.0001 & \pm 0 & \pm 0 \\
$\mathcal{A}_{\mathrm{b}}$ & 0.9343 & \pm 0 & $\mp 0.0005$ & $\mp 0.0002$ \\
$\mathcal{A}_{\mathrm{c}}$ & 0.6658 & \pm 0.0006 & $\mp 0.0025$ & $\mp 0.0008$ \\
\hline
\end{tabular}

Table 3: Standard Model predictions for the electroweak observables with quarks for different input parameters.

\section{LEP and SLC}

The $\mathrm{e}^{+} \mathrm{e}^{-}$storage rings LEP at CERN [24] and SLC at SLAC [25] were designed to accelerate particles to centre of mass energies of larger than $90 \mathrm{GeV}$ to produce large samples of $\mathrm{Z}$ bosons.

LEP is a circular machine and delivered between 1989 and 1995 approximately four million $\mathrm{Z}$ bosons to each of the experiments. Since 1996 it is running at higher energies to produce W pairs and search for new particles. At LEP there are four large detectors, ALEPH [26, 27], DELPHI [28,29], L3 [30] and OPAL [31], all running since 1989.

SLC is a linear collider where electrons and positrons are accelerated in the same structure. At the end of the linear accelerator (linac) they are guided through two different arcs and brought into collision in one interaction region. Due to the linac technology, SLC is able to produce polarised electron beams with about $80 \%$ longitudinal polarisation at the interaction point. This allows additional observables to be measured, as explained in section 2 . In addition, the beam pipe inside the experiment and the size of the beam spot are both much smaller than at LEP, which allows a more efficient tagging of b-quarks. Up to the end of 1997, SLD recorded $300000 \mathrm{Z}$ bosons. However, in many analyses the small statistics is compensated by the beam polarisation and the small beam and beampipe size.

SLC started in 1989 with an upgraded version of the Mark II detector [32]. However, since no results of this detector are used here, it will not be described further. In November 1990 Mark II was replaced by the SLD detector [33] which is taking data since 1992.

All detectors are built with a more or less cylindrical symmetry around the beampipe and have a solenoidal magnetic field to measure charged particle momenta. Fig. 3 shows the DELPHI detector as one example. 
In all cases the beampipe is surrounded by several layers of very precise silicon detectors [34-38] to measure secondary vertices and to tag $\mathrm{Z} \rightarrow \mathrm{b} \overline{\mathrm{b}}$ events. They are followed by a set of gaseous drift chambers to measure the momenta of charged particles. The tracking system is surrounded by the electromagnetic calorimeters. These detectors are split into a cylindrical barrel and two plane endcaps. To improve the energy and position resolution all detectors apart from OPAL have the electromagnetic calorimeters inside the coil. Behind the electromagnetic calorimeters the experiments have the hadron calorimeters. Apart from L3, they consist of instrumentation inside the iron yoke that returns the magnetic field. The detectors are completed by another set of drift chambers outside the iron to identify muons.

Charged hadrons can be identified by the specific energy loss in the central drift chambers in ALEPH, DELPHI and OPAL and with ring imaging Cherenkov counters in DELPHI and SLD.

The luminosity is measured by counting the number of Bhabha events, $\mathrm{e}^{+} \mathrm{e}^{-} \rightarrow \mathrm{e}^{+} \mathrm{e}^{-}$, at small angles for which the cross section can be calculated reliably in QED. The differential cross section is proportional to $1 / \theta^{3}$. For a high event rate it is thus important to measure at very low scattering angles. To obtain a high precision on the luminosity, primarily a good definition of the inner acceptance border is needed.

For these reasons, all experiments have a special electromagnetic calorimeter surrounding the beampipe at distances of about $2 \mathrm{~m}$ away from the interaction point. They typically cover the angular range 30 to $180 \mathrm{mrad}$, resulting in visible cross sections of more than $40 \mathrm{pb}$.

Table 4 compares the performances of the five experiments. For the physics discussed here the performance of the detectors is similar with the exception of SLD's vertex detector performance. Due to the small beampipe their extrapolation distance is much smaller than at LEP, and due to the pointlike beamspot there is no need for a primary vertex fit. In addition the low repetition rate $(120 \mathrm{~Hz})$ of the linac allows the use of CCD pixel detectors, which cannot be used at LEP with a $40 \mathrm{kHz}$ bunch crossing rate.

\subsection{Lepton identification}

Muons are identified by the matching of a track in the inner tracking system with hits in the Muon chambers. In ALEPH, DELPHI, OPAL and SLD the muon chambers are located outside the solenoid and serve only as identification devices, while the L3 muon system is located inside the coil and measures the muon momentum more precisely than the inner tracker. In all cases, hadrons are absorbed in the electromagnetic and hadronic calorimeters which have a total thickness of typically seven hadronic interaction lengths. The muon identification can be improved by requiring that the energy deposit in the hadron calorimeter is consistent with a minimum ionising particle. For a muon identification efficiency of $85 \%$, the misidentification probability for charged hadrons is typically around $1 \%$.

The muon identification efficiency can be measured with high precision for $p_{\mu} \approx 45 \mathrm{GeV}$ using $\mathrm{Z} \rightarrow \mu^{+} \mu^{-}$events, and the energy dependence with less statistics using $\mathrm{Z} \rightarrow \tau^{+} \tau^{-}$events with $\tau \rightarrow \mu \nu \nu$.

For the simulation of the hadron misidentification rate, a detailed understanding of the development of hadronic showers is needed. For this all experiments use the GEANT detector simulation package [39]. As an additional complication, the cross sections for interaction with matter are different for $\pi^{ \pm}, \mathrm{K}^{+}$and $\mathrm{K}^{-}$. The misidentification rate can, however, be measured using e.g. hadrons from $\mathrm{K}_{\mathrm{S}}^{0} \rightarrow \pi^{+} \pi^{-}$or $\mathrm{D}^{*+} \rightarrow \pi^{+} \pi^{+} \mathrm{K}^{-}$decays ${ }^{3}$.

\footnotetext{
${ }^{3}$ If not explicitly mentioned charge conjugate states are always implicitly included.
} 


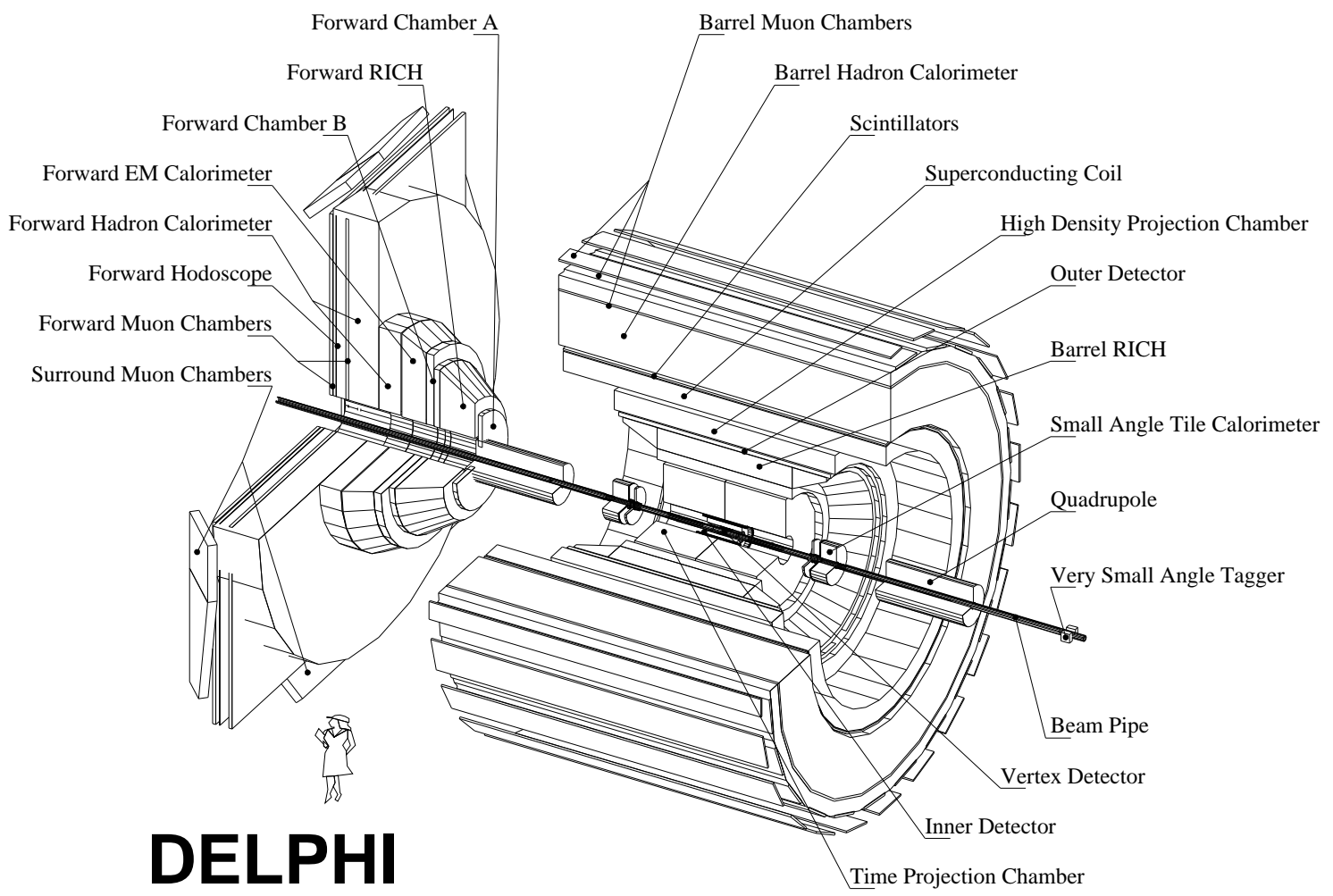

Figure 3: The DELPHI detector at LEP.

\begin{tabular}{|l|c|c|c|c|c|}
\hline & ALEPH & DELPHI & L3 & OPAL & SLD \\
\hline \hline B-field & $1.5 \mathrm{~T}$ & $1.2 \mathrm{~T}$ & $0.5 \mathrm{~T}$ & $0.435 \mathrm{~T}$ & $0.6 \mathrm{~T}$ \\
\hline$\sigma(1 / p) \times$ & 0.6 & 0.6 & $\mu: 0.6$ & 1.5 & 2.6 \\
$\frac{10^{-3}}{\sqrt{\mathrm{GeV}}}$ & & & $h: 1.8$ & & \\
\hline$\frac{\sigma(E)}{E}(\mathrm{EM})$ & $\frac{0.18}{\sqrt{E \mathrm{GeV}}} \oplus 0.01$ & $\frac{0.32}{\sqrt{E \mathrm{GeV}}} \oplus 0.04$ & $\frac{0.02}{\sqrt{E \mathrm{GeV}}} \oplus 0.01$ & $\frac{0.06}{\sqrt{E \mathrm{GeV}}} \oplus 0.02$ & $\frac{0.15}{\sqrt{E \mathrm{GeV}}}$ \\
\hline$\frac{\sigma(E)}{E}(\mathrm{Had})$ & $\frac{0.85}{\sqrt{E \mathrm{GeV}}}$ & $\frac{1.12}{\sqrt{E \mathrm{GeV}}} \oplus 0.21$ & $\frac{0.55}{\sqrt{E \mathrm{GeV}}} \oplus 0.08$ & $\frac{\sim 1}{\sqrt{E \mathrm{GeV}}}$ & $\frac{0.60}{\sqrt{E \mathrm{GeV}}}$ \\
\hline$R \Phi$ IP resol. & $25 \mu \mathrm{m}$ & $20 \mu \mathrm{m}$ & $30 \mu \mathrm{m}$ & $18 \mu \mathrm{m}$ & $11 \mu \mathrm{m}$ \\
\hline$z$ IP resol. & $25 \mu \mathrm{m}$ & $30 \mu \mathrm{m}$ & $30 \mu \mathrm{m}$ & $20 \mu \mathrm{m}$ & $22 \mu \mathrm{m}$ \\
\hline
\end{tabular}

Table 4: Comparison of the performances of the LEP and SLC detectors. The numbers are given for $\theta=90^{\circ}$ including the latest upgrades. (IP $=$ impact parameter) 
Electrons are identified mainly by matching a charged particle track with a shower in the electromagnetic calorimeter. The matching is done in space and energy, and the shower shape has to be compatible with the one expected for electrons. Additional information can be obtained from the specific energy loss $(\mathrm{dE} / \mathrm{dx})$ in the central tracking detectors. Combining both methods, typical efficiencies around $70 \%$ can be reached for misidentification probabilities of a few permille. Both identification methods work better for isolated tracks, so that the efficiency and purity normally increases for tracks with large transverse momentum. Efficiency and background can be measured as for muons. Since the two electron identification methods are independent, they can be calibrated by comparing the calorimetric and $\mathrm{dE} / \mathrm{dx}$ response to single tracks.

\section{$4 \quad$ Flavour tagging methods}

A couple of distinct features allow an efficient tagging of b and c quarks:

- Weakly decaying b- and c-hadrons have lifetimes of the order of picoseconds. Due to their large boost they fly around $1 \mathrm{~mm}$ before they decay and their decay particles can be tagged by the silicon microvertex detectors.

- b- and c-hadrons decay semileptonically with branching ratios of about $10 \%$ per lepton species.

- c-hadrons can be reconstructed fully in several decay modes with reasonable efficiencies. Since b-hadrons decay mostly via c-hadrons, this method tags b-quarks as well.

The separation of b- and c-quarks is done using the mass difference between b- and c-hadrons in the case of lifetime and lepton tags. The mass of the decaying particle is either estimated directly or secondary features of the decay products like their transverse momentum, impact parameter or multiplicity are used. In the case of full $\mathrm{D}$ reconstruction, the separation is either done by the c-hadron energy or using other features of the event like lifetime.

Depending on the analysis the tagging can be done on the full event, a single hemisphere in the event or a jet. Performing the tagging on the full event usually gives the best efficiency for a given purity. Separating the event into two hemispheres, usually with respect to the thrust axis, and running the tagging algorithm separately on the hemispheres allows to extract the tagging efficiency directly from the data, as explained in section 5.2, or to analyse the hemisphere opposite to the tagged one in an unbiased way. For some analyses, like the measurements of gluon splitting rates, one even has to run first a jet algorithm on all particles in the event and then perform the tagging on the different jets.

\subsection{Lepton tags}

As mentioned above, b- and c-hadrons decay semileptonically with branching ratios of the order of $10 \%$. Due to momentum conservation the transverse momentum $\left(p_{t}\right)$ of the lepton with respect to the b- or c-hadron direction is limited to half the hadron mass. Since the hadron direction is typically well approximated by the direction of the jet containing the hadron, the transverse momentum of the lepton with respect to the jet is a powerful separator between leptons from $\mathrm{b}$ - and c-quarks. In addition, leptons from the decay chain $\mathrm{b} \rightarrow \mathrm{c} \rightarrow \ell$ have on average a much smaller $p_{t}$ than the prompt leptons from $\mathrm{b} \rightarrow \ell$ and the $p_{t}$ of misidentified 
hadrons is typically only a few hundred $\mathrm{MeV}$, so that a simple cut on $p_{t}$ provides a quite pure $\mathrm{b} \rightarrow \ell$ sample.

On the other hand there is no region in $p, p_{t}$ where leptons from $\mathrm{c} \rightarrow \ell$ decays are clearly separated from $\mathrm{b} \rightarrow \mathrm{c} \rightarrow \ell$ and misidentified hadrons. However, when the shapes of the spectra are known or when charm is enriched by other means, analyses on a statistical basis are possible.

As an example, figure 4 shows the $p$ and $p_{t}$ spectra from L3 together with the Monte Carlo predictions for the different sources.
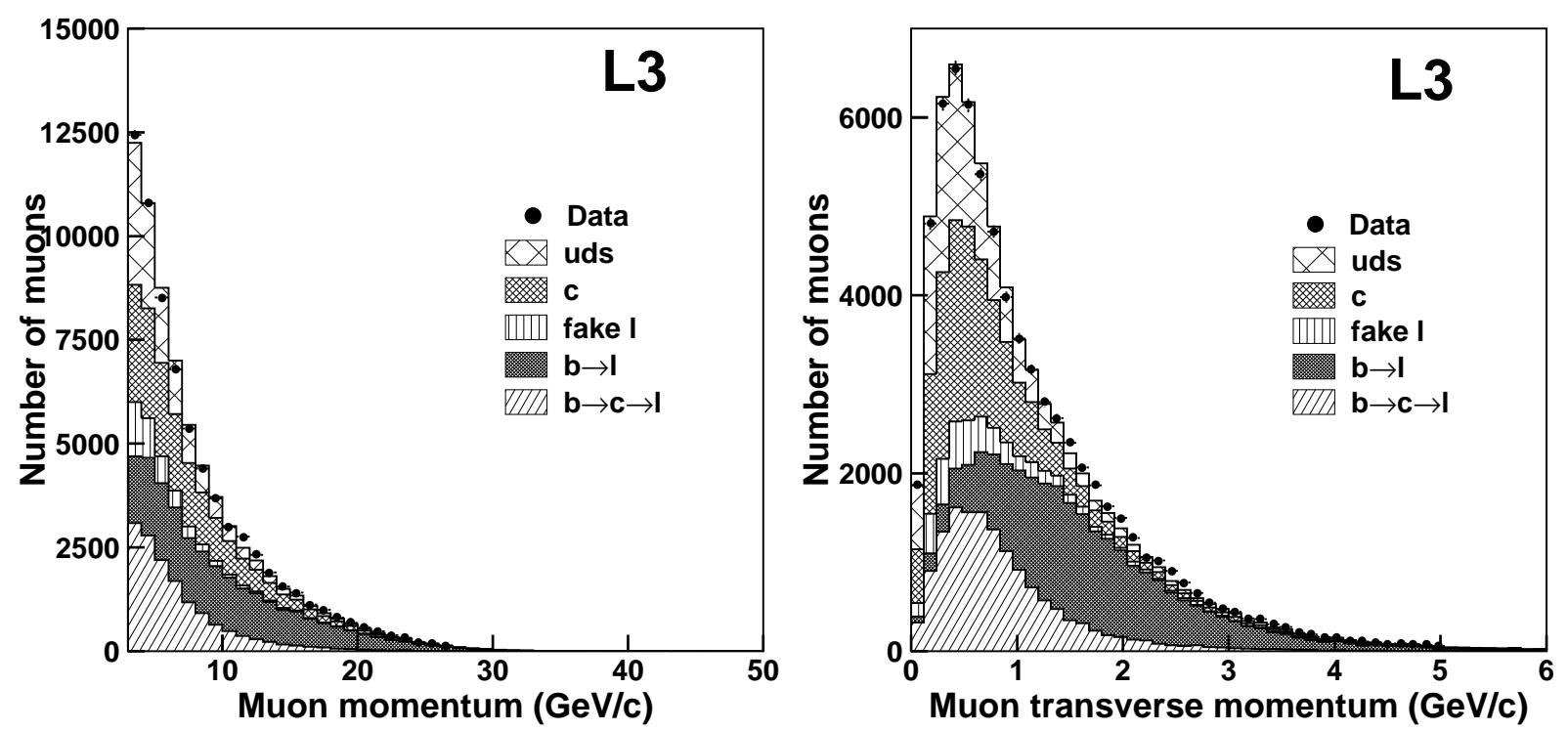

Figure 4: Muon momentum $(p)$ and transverse momentum $\left(p_{t}\right)$ spectra for data and the Monte Carlo predictions for the different sources obtained from L3.

OPAL uses an enhanced lepton tag including additional hadronic variables from the jet that contains the lepton processed by a neural net [40]. The resulting network output for $\mathrm{b} \rightarrow \ell$ and $\mathrm{c} \rightarrow \ell$ decays is shown in figure 5 . The improvement compared to the pure lepton tag is modest for $\mathrm{b} \rightarrow \ell$, but quite large for $\mathrm{c} \rightarrow \ell$.

\subsection{Lifetime tags}

The by far most efficient and pure b-tags are the lifetime tags. Due to the long lifetime and high mass of b-hadrons B decay particles have typical impact parameters ${ }^{4} \delta$ of around $150 \mu \mathrm{m}$ compared to a resolution of around $50 \mu \mathrm{m}$ at $\mathrm{LEP}^{5}$ and $15 \mu \mathrm{m}$ at SLC. The experiments either use the presence of large impact parameters to tag b events directly, or they try to fit secondary vertices. Since D-mesons and B-mesons have similar lifetimes, some residual charm background is unavoidable in pure lifetime tags. This background can, however, be suppressed by a cut on the invariant mass of the secondary particles.

\footnotetext{
${ }^{4}$ The impact parameter $\delta$ is defined as the closest distance of the particle trajectory to the $\mathrm{Z}$ decay point.

${ }^{5}$ Contrary to table 4 this number contains the contribution from the primary vertex error.
} 

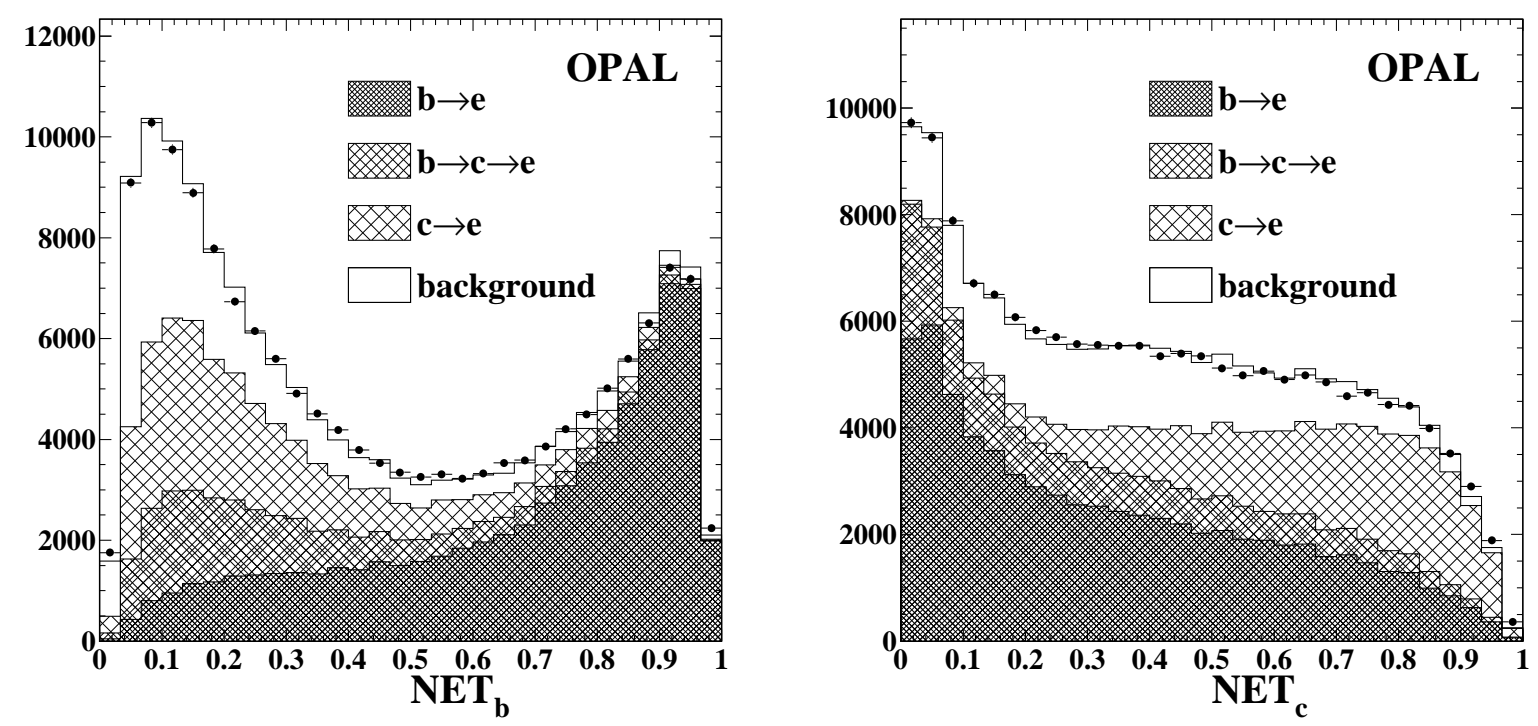

Figure 5: Neural network output for the OPAL electron b- and c-tag.

\subsubsection{Impact parameter tags}

ALEPH [41], DELPHI [42] and L3 [43] use a tag mainly based on impact parameters. The algorithm usually defines first a probability, based on the impact parameter significance ${ }^{6}$, that a track originates from the primary vertex. By definition, this probability is flat for primary tracks and peaked at low values for b-decay products. For any group of tracks, these probabilities can be combined into a probability that all tracks of this group come from the primary vertex, which is again flat for light quark events and peaked at low values for b-events. Depending on the analysis this probability can be calculated for all tracks in a jet, a hemisphere or a whole event.

Although all experiments are equipped with vertex detectors that provide three dimensional information, the impact parameter resolution and association efficiency is typically different in the $r-\Phi$ and $r-z$ projection. For that reason some experiments measure the impact parameter separately in the two projections and combine them later in the probability calculation.

To measure the experimental resolution from the data themselves the impact parameter is signed. In the two dimensional case $\delta$ is signed positive if the track crosses the jet axis in the direction of its flight seen from the primary vertex, and negative otherwise. In the three dimensional case the point of smallest distance between the track and the jet axis is used instead. If the jet direction represents the flight direction of the decaying particle a true impact parameter normally has to be positive. The negative part of the impact parameter distribution is thus a measure of the experimental resolution including wrongly associated hits in the microvertex detector. This is demonstrated in figure 6 a) which shows the impact parameter significance distribution obtained by ALEPH for data and simulation [41].

\subsubsection{Vertex tags}

OPAL [44] and SLD [45] use secondary vertices to tag b $\bar{b}$ events. In this case the distance between the primary and the secondary vertex measured along the direction of the jet containing

\footnotetext{
${ }^{6}$ The impact parameter significance is defined as the impact parameter divided by its error.
} 

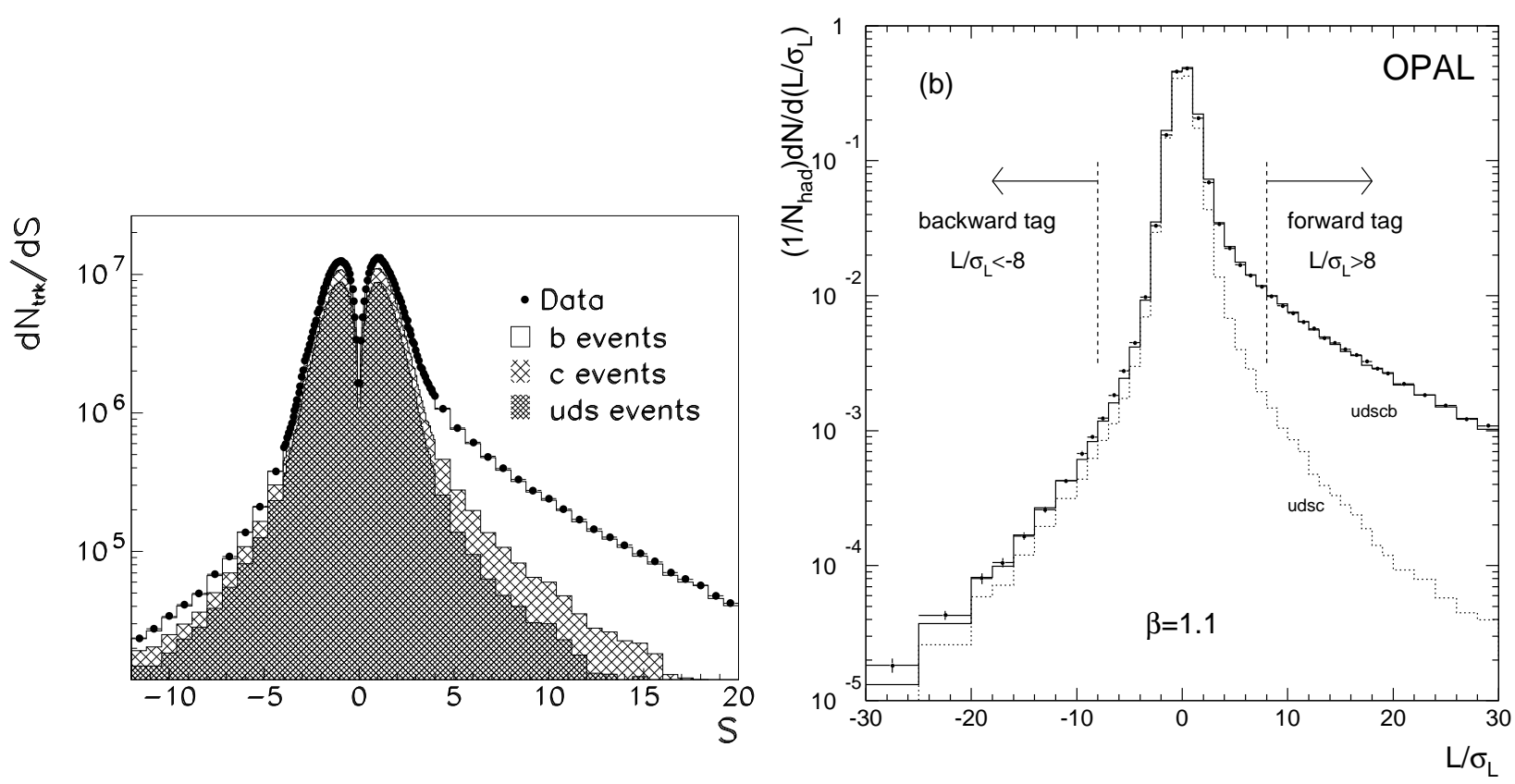

Figure 6: a) Impact parameter significance distribution from ALEPH for data and simulation, b) Secondary vertex decay length distribution from OPAL. The parameter $\beta$ indicates the additional smearing that has to be applied to reach data - Monte Carlo agreement.

the vertex, divided by its error is used as a b-tag. The sign of the decay distance is positive when the vertex is in the hemisphere of the decay particles and negative otherwise. Figure 6 b) shows the normalised decay distance distribution for data and simulation from OPAL.

\subsubsection{Mass tags}

Using pure impact parameter or vertex tags, a residual charm background is difficult to reject since $b$ and $c$ hadrons have comparable lifetimes. A natural way to reject this background is to cut on the mass of the decaying particle.

For secondary vertices, a mass cut can naturally be applied to the invariant mass of the particles attached to the vertex. Because of the small beampipe and especially the point-like beamspot, SLD also has a very good resolution on the direction of the decaying particle. Using the transverse momentum of the particles attached to the secondary vertex with respect to the direction from the primary to the secondary vertex, an additional correction can even be applied for the mass carried away by neutral particles.

As can be seen from figure $7 \mathrm{a}$ ), an almost pure b-sample can be obtained by requiring this mass to be larger than $1.8 \mathrm{GeV}$. With the good mass resolution at SLD it is even possible, using this method, to tag charm with reasonable efficiency and purity by requiring the reconstructed mass to be below the charm mass. For b-hemispheres, the reconstructed mass can be below the charm mass if enough B-decay particles are lost. But the reconstructed momentum is then also much smaller than the true B-momentum. The reconstructed momentum of a vertex of a given mass is therefore on average much smaller for a b-hemisphere than for a c-hemisphere. As one can see from figure 8, this feature can be used to enhance the purity of the charm tag significantly.

DELPHI also uses the invariant mass of a reconstructed secondary vertex combined in a 
probabilistic way with the impact parameter tag. The rapidities of the secondary particles and the energy of all particles fitted to the decay vertex are also used. Figure $7 \mathrm{~b}$ ) shows the improvement in the efficiency-purity curve due to this additional information.
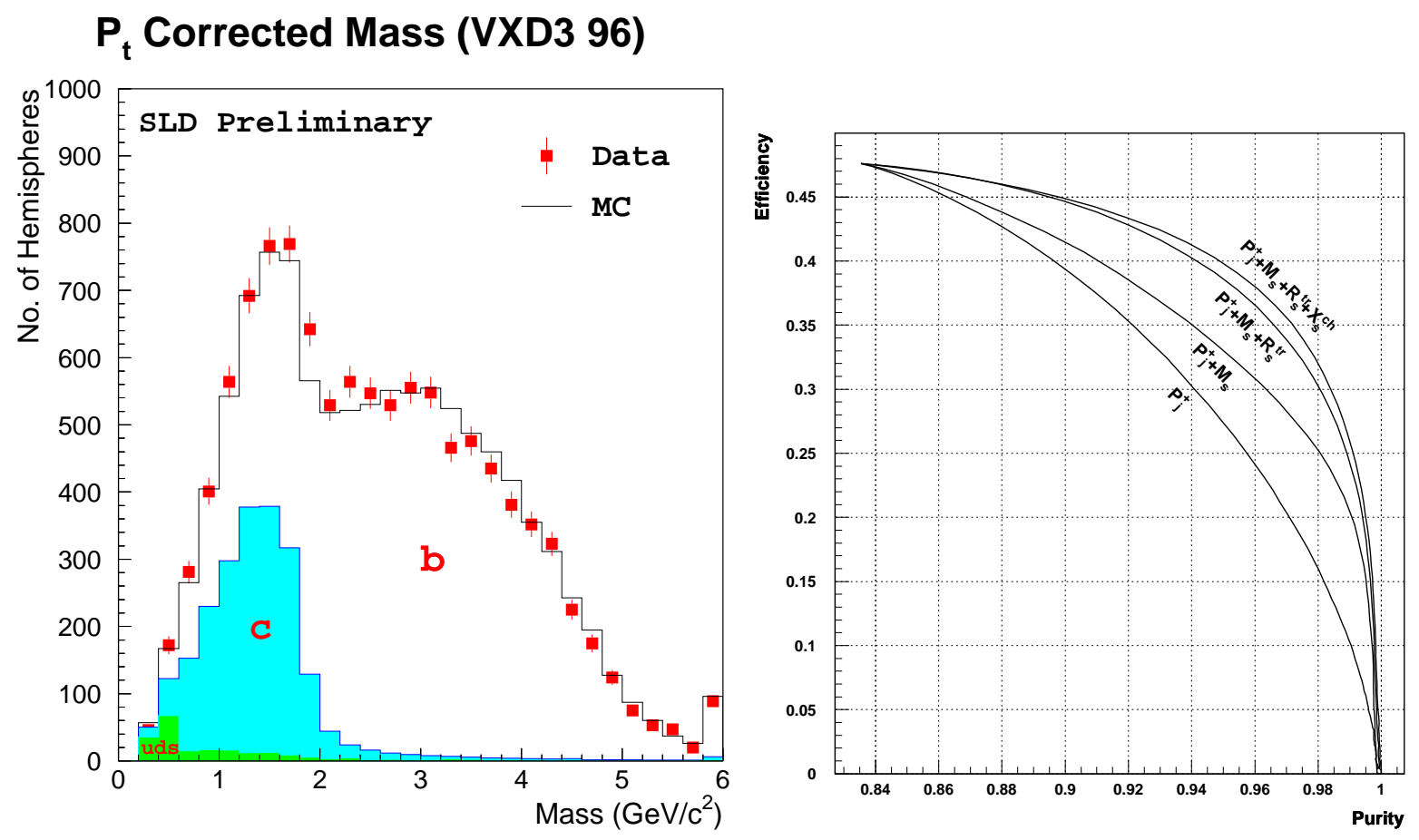

Figure 7: a) $p_{t}$ corrected invariant mass distribution of secondary vertices from SLD,

b) Efficiency purity curve from DELPHI for the hemisphere-tag using only impact parameters and after including the mass, rapidities and total energy of the tracks from the secondary vertex.

ALEPH orders the particles in a hemisphere with decreasing impact parameter significance. The invariant mass of the first particles in this order is calculated and the impact parameter significance for the particle whose addition causes the mass to exceed the D-mass is used as an additional tag. This tag reaches a performance similar to the one used by DELPHI.

\subsection{D-Meson tags}

Since the production of charm quarks in fragmentation is heavily suppressed, the presence of charmed hadrons tags $c \bar{c}$ and $b \bar{b}$ events. The $b-$ and c-events must then be separated with other methods. The cleanest tag is the reconstruction of the decay chain $\mathrm{D}^{*+} \rightarrow \pi^{+} \mathrm{D}^{0}$ with $\mathrm{D}^{0} \rightarrow \mathrm{K}^{-} \pi^{+}$or $\mathrm{D}^{0} \rightarrow \mathrm{K}^{-} \pi^{-} \pi^{+} \pi^{+}$. The small difference between the $\mathrm{D}^{*+}$ mass and the sum of the $\mathrm{D}^{0}$ and pion masses can be measured with very good resolution, leading to a good signal to background ratio. Figure 9 shows the mass- and mass difference spectra obtained by DELPHI for $\mathrm{D}^{0} \rightarrow \mathrm{K}^{-} \pi^{+}$[46]. However due to the small branching ratios involved, the efficiency of this channel for c-tagging is rather small.

Depending on the analysis several other charmed hadron tags are used:

- exclusive reconstruction of weakly decaying charmed hadrons, 

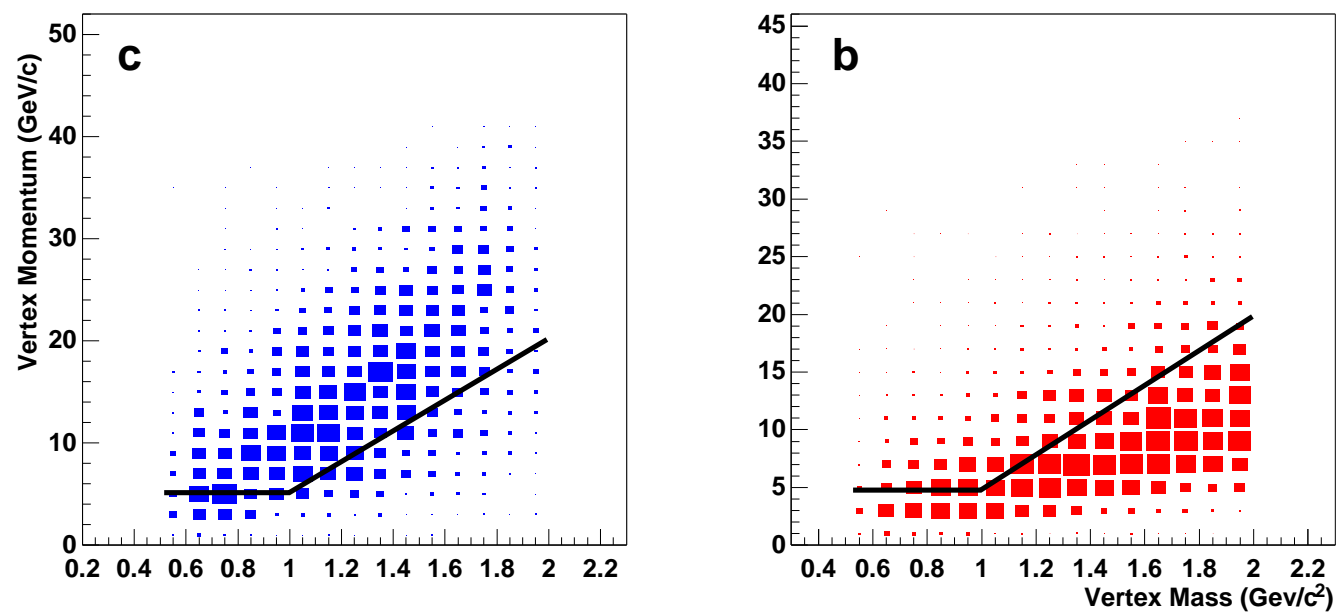

Figure 8: Reconstructed momentum versus invariant mass for vertices reconstructed by SLD. The left plot is for hemispheres containing a charm quark and the right plot for those containing a b quark. The line indicates the cut used in the $R_{\mathrm{c}}$ analysis.

\section{DELPHI}
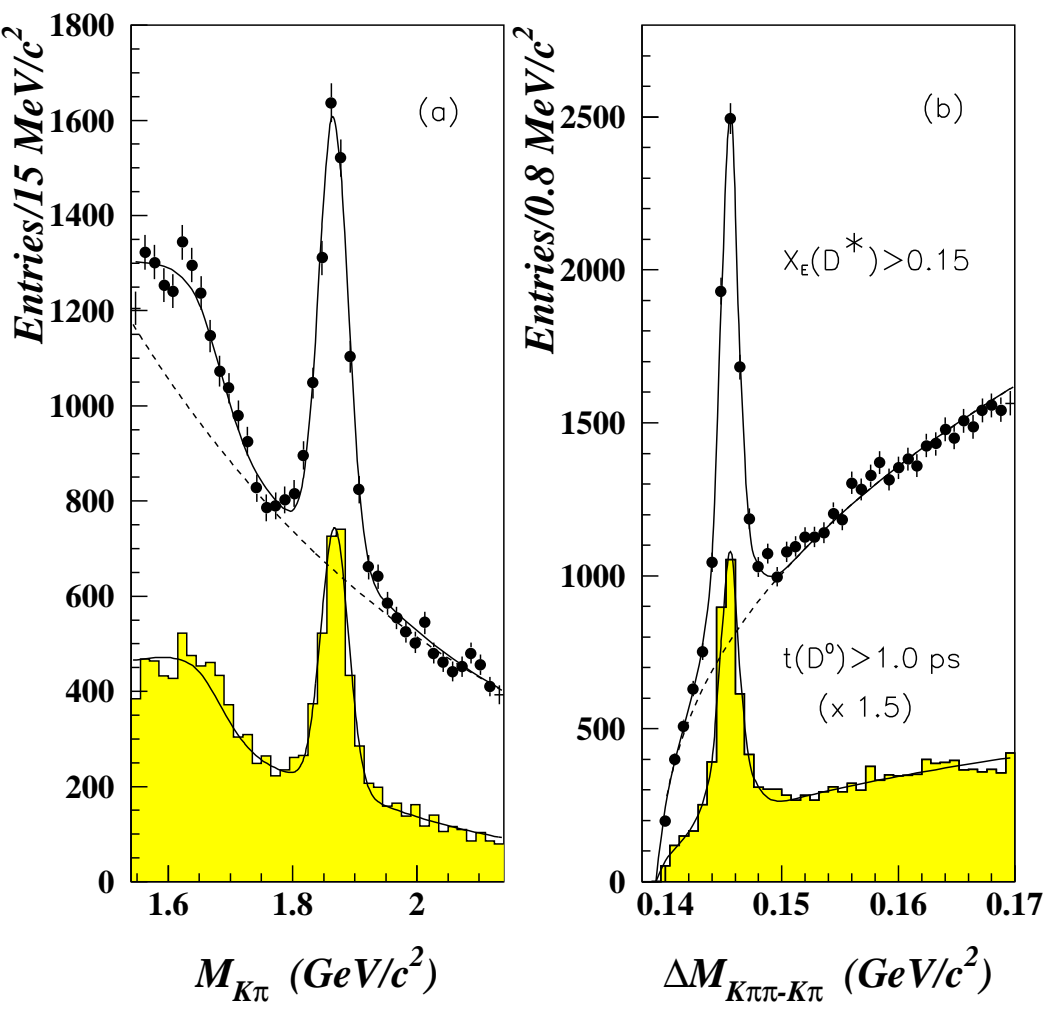

Figure 9: $\mathrm{m}\left(\mathrm{K}^{-} \pi^{+}\right)$mass spectrum (a) and $\mathrm{m}\left(\mathrm{K}^{-} \pi^{+}\right)-\mathrm{m}\left(\mathrm{K}^{-} \pi^{+} \pi^{+}\right)$mass difference spectrum (b) from DELPHI. Each plot contains a cut around the peak of the other one. 
- semi-exclusive $\mathrm{D}^{*+}$ reconstruction,

- inclusive $\mathrm{D}^{*+}$ tagging.

\subsubsection{Exclusive reconstruction of weakly decaying charmed hadrons}

$\mathrm{D}^{0}, \mathrm{D}^{+}, \mathrm{D}_{\mathrm{s}}$ and $\Lambda_{\mathrm{c}}$ can be reconstructed at LEP in channels with well known branching ratios. Figure 10 shows the mass spectra for these four hadrons obtained by ALEPH [47]. As can be seen, the high background does not allow a pure event by event tagging. However, fitting the mass spectrum allows a precise estimate of the signal. The production rates of weakly decaying charmed hadrons in $\mathrm{b} \overline{\mathrm{b}}$ and $c \overline{\mathrm{c}}$ events are interesting in themselves. In addition they are used for measurements of $R_{\mathrm{c}}$ and for the charm forward-backward asymmetry.

\subsubsection{Semi-exclusive $D^{*+}$ reconstruction}

Despite to the poorer mass resolution, the mass difference method can also be used even if the $\mathrm{D}^{0}$ is only partially reconstructed. Mostly the two decay modes $\mathrm{D}^{0} \rightarrow \mathrm{K}^{-} \pi^{+} \pi^{0}$ and $\mathrm{D}^{0} \rightarrow \mathrm{K}^{-} \ell \nu$ are used for this. In the mode $\mathrm{D}^{0} \rightarrow \mathrm{K}^{-} \pi^{+} \pi^{0}$, the $\pi^{0}$ is usually not reconstructed. However, the decay goes dominantly via $\mathrm{D}^{0} \rightarrow \mathrm{K}^{-} \rho^{+}$, with the $\rho$ being strongly polarised. As can be seen from figure 9 , this leads to a strong satellite peak in the $\mathrm{K}^{-} \pi^{+}$mass spectrum which can be used for the mass difference calculation. In the mode $\mathrm{D}^{0} \rightarrow \mathrm{K}^{-} \ell \nu$, lepton identification suppresses the backgrounds sufficiently, that the mass difference trick allows a reasonably pure tagging. As there is a relatively large background from $\mathrm{D}^{*+} \rightarrow \pi^{+} \mathrm{D}^{0}$ decays where the $\mathrm{D}^{0}$ decays into channels other than the selected one, this method is not used for rate measurements. However for asymmetry measurements, where an exact knowledge of the efficiency is not needed, these channels are still useful.

\subsubsection{Inclusive $\mathrm{D}^{*+}$ tagging}

The transverse momentum $\left(p_{t}\right)$ of particles due to the fragmentation process depends only weakly on the momentum of the particle and is of the order of $100 \mathrm{MeV}$. The $p_{t}$ of a $\mathrm{D}^{*+}$ with respect to the jet axis is thus comparable to the $p_{t}$ of fragmentation pions. Due to the small $Q$-value of the $\mathrm{D}^{*+} \rightarrow \pi^{+} \mathrm{D}^{0}$ decay and the large $\pi^{+}-\mathrm{D}^{0}$ mass difference, the $\pi^{+}$follows closely the $\mathrm{D}^{*+}$ flight direction and has low momentum. Its $p_{t}$ is thus much smaller than the average $p_{t}$ from fragmentation pions. By fitting the $p_{t}$ distribution at larger $p_{t}$ and extrapolating the fit to small $p_{t}$, the number of events containing a $\mathrm{D}^{*+} \rightarrow \pi^{+} \mathrm{D}^{0}$ can be deduced. In addition the momentum of the pion is strongly correlated to that of the $\mathrm{D}^{*+}$. Figure 11 shows the $p_{t}$ spectra opposite to a high energy $\mathrm{D}^{* \pm}$ for pions with the opposite (a) and same (b) sign as the $\mathrm{D}^{* \pm}$ [48]. For the opposite sign, where a $\mathrm{D}^{* \pm}$ signal is expected, a clear enhancement at low $p_{t}$ can be seen.

\subsection{4 b-c separation}

A model-independent b-c separation can be done using lifetime information. For this, either the reconstructed charmed hadrons themselves or the algorithms described above are used. The latter can be applied in the hemisphere opposite to the one with the tagged charmed hadron, so that an unbiased analysis is possible. 

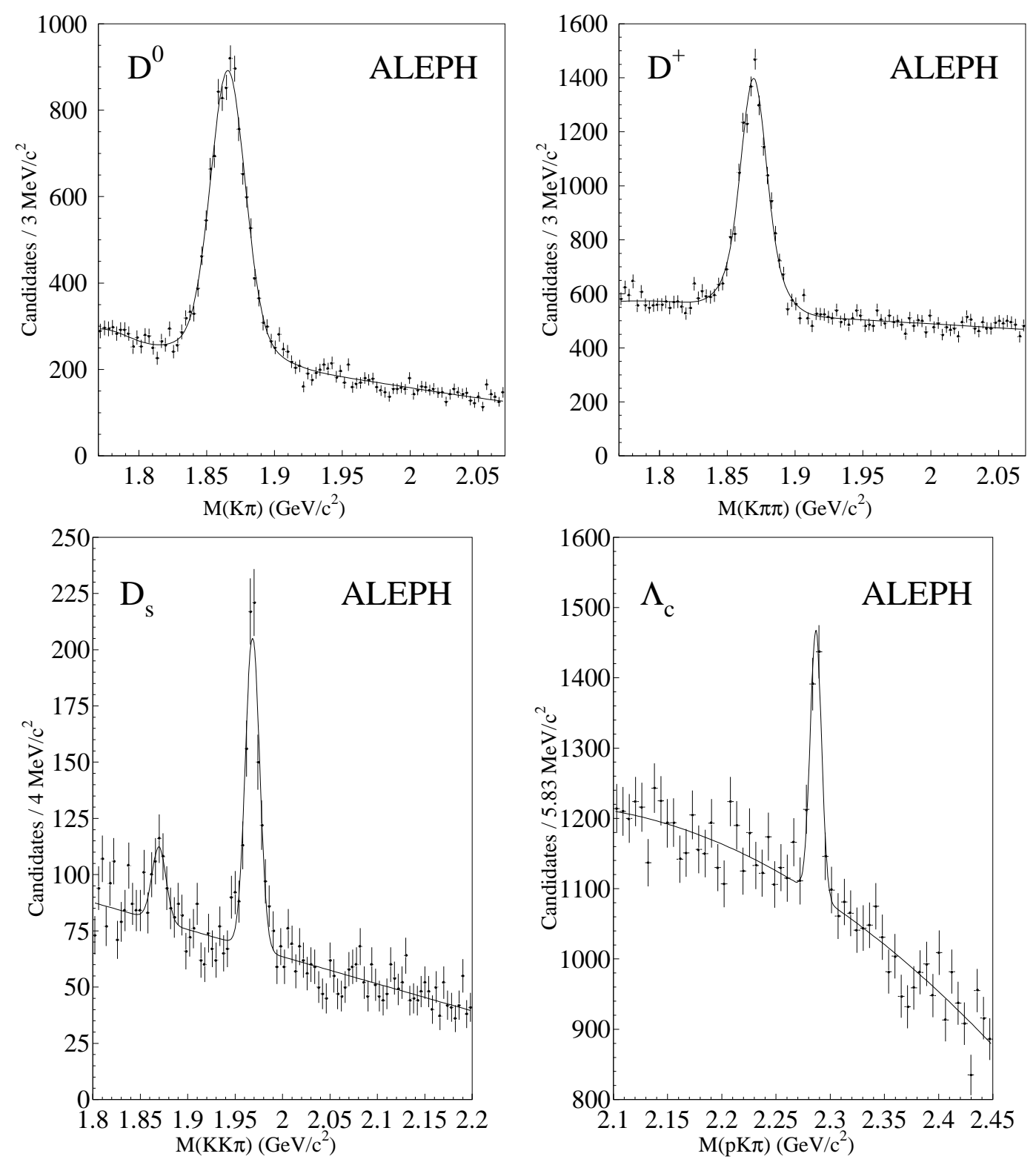

Figure 10: Mass spectra for $\mathrm{D}^{0} \rightarrow \mathrm{K}^{-} \pi^{+}, \mathrm{D}^{+} \rightarrow \mathrm{K}^{-} \pi^{+} \pi^{+}, \mathrm{D}_{\mathrm{s}} \rightarrow \mathrm{K}^{+} \mathrm{K}^{-} \pi^{+}$and $\Lambda_{\mathrm{c}} \rightarrow \mathrm{p} K^{-} \pi^{+}$ obtained by ALEPH. 

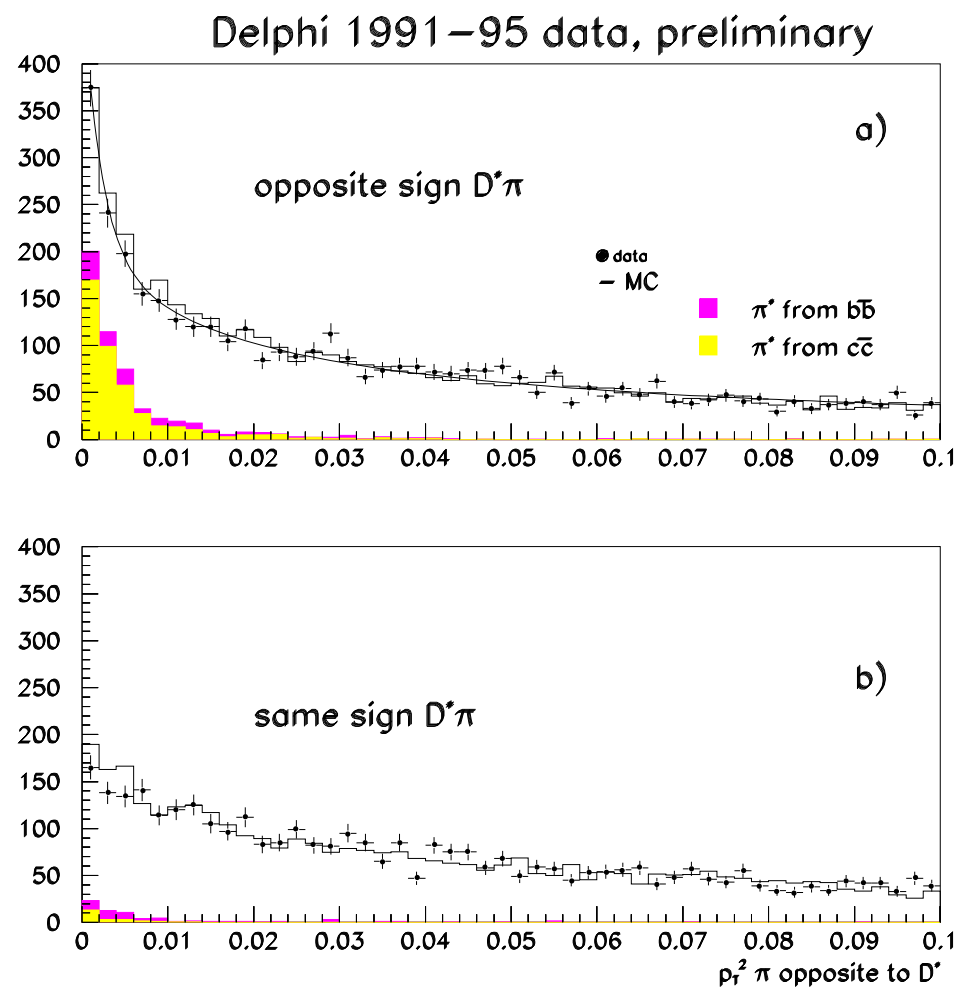

Figure 11: $p_{t}$ spectrum opposite to a high energy $\mathrm{D}^{* \pm}$ for pions with the opposite (a) and same (b) sign as the $\mathrm{D}^{* \pm}$. The low background under the signal in a) is due to the charm tag in the opposite hemisphere.

Figure 12 shows the momentum spectrum of $\mathrm{D}^{*+}$ mesons from OPAL using lifetime tagging in both hemispheres, jetshapes in the opposite hemisphere and the jetcharge in the $\mathrm{D}^{* \pm}$ hemisphere [49]. $\mathrm{D}^{* \pm}$ from charm are on average much faster than those from $b$. Once the momentum spectra for charmed hadrons are known, b-c separation can be done efficiently using the charmed hadron momentum.

\subsection{Other tagging methods}

At lower energy machines like PEP and PETRA, efficient b-tagging could be obtained using event shape distributions. At the higher energy, the separation power of these methods has diminished significantly. This can be recovered partially by combining many quantities in a neural network. However the weight of these techniques in the latest analyses is minor, so they will not be described further.

\section{Measurements of the electroweak quantities}

\section{$5.1 \quad$ Asymmetry measurements}

The main methods used for the asymmetry measurements are:

- lepton measurements for $A_{\mathrm{FB}}^{\mathrm{b}}$ and $A_{\mathrm{FB}}^{\mathrm{c}}$, 


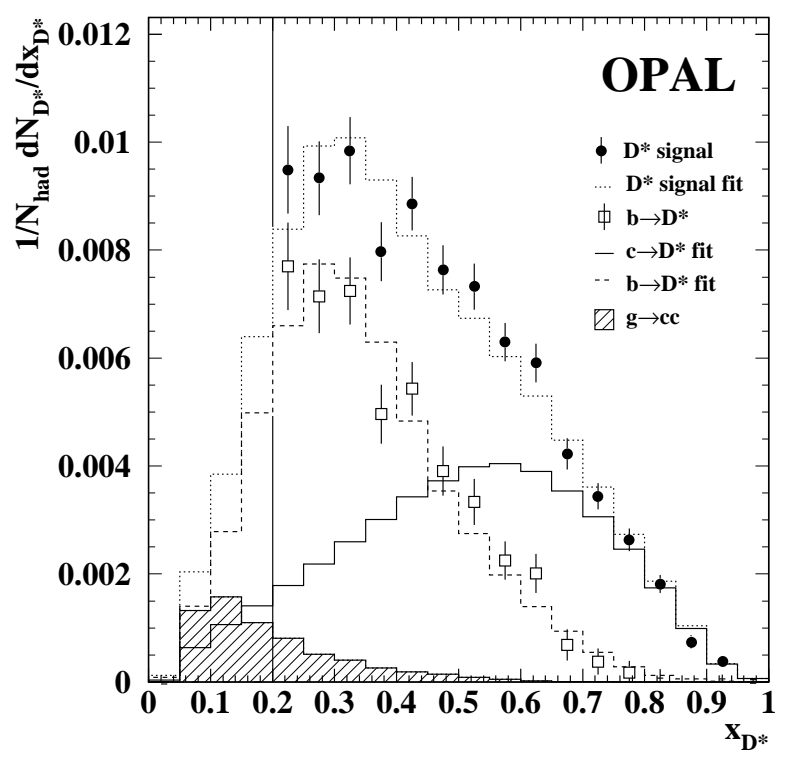

Figure 12: $D^{* \pm}$ momentum spectrum for all events and for $b \bar{b}$ and $c \bar{c}$ events from OPAL normalised to the beam energy after subtraction of combinatorial background.

- D-Mesons for $A_{\mathrm{FB}}^{\mathrm{c}}$,

- charge flow measurements with lifetime tags for $A_{\mathrm{FB}}^{\mathrm{b}}$,

- charged Kaons for $A_{\mathrm{FB}}^{\mathrm{b}}$.

To measure a quark asymmetry, a pure sample of $q \bar{q}$ events needs to be prepared and the direction of the fermion has to be estimated. As estimator for the quark direction always the thrust axis is used. The sign ambiguity is solved by projecting the tagging particle onto the thrust axis. In most analyses, the asymmetry is obtained by fitting the differential asymmetry as a function of $\cos \theta_{\text {thrust }}$. This is not only statistically more powerful than counting the total numbers of forward and backward events, but also cancels some systematic uncertainties like the polar angle dependence of the acceptance. As an example figure 13 shows the signed $\cos \theta_{\text {thrust }}$ distribution from the ALEPH lepton analysis [50] where the asymmetry can clearly be seen.

\subsubsection{Lepton measurements}

As explained in section 4.1, the presence of a lepton tags $\mathrm{b}$ and $\mathrm{c}$ events. Especially in $\mathrm{b} \overline{\mathrm{b}}$ events, leptons can originate from several sources with different charge correlations to the decaying quark, as shown in table 5 .

Due to $\mathrm{B}^{0} \overline{\mathrm{B}^{0}}$ mixing, the asymmetry is diluted by a factor $(1-2 \bar{\chi})$, where $\bar{\chi}$ is the average mixing parameter for the events in the sample. As seen from figure 4 , the leptons from $\mathrm{b} \rightarrow \ell$ can be separated efficiently using their transverse momenta. This is illustrated in table 5 by the sample composition in the ALEPH analysis after a cut $p_{t}>1.25 \mathrm{GeV}$.

ALEPH [50] and L3 [51] measure $A_{\mathrm{FB}}^{\mathrm{b}}$ alone from a sample of high $p_{t}$ leptons. DELPHI [52], OPAL [53], and SLD [54] fit to the full $\left(p, p_{t}\right)$ spectra or, in the case of OPAL, the neural net output to obtain $A_{\mathrm{FB}}^{\mathrm{b}}$ and $A_{\mathrm{FB}}^{\mathrm{c}}$. The corrections for the sample composition are always done using the simulation. However the branching ratios $\mathrm{BR}(\mathrm{b} \rightarrow \ell), \mathrm{BR}(\mathrm{b} \rightarrow \mathrm{c} \rightarrow \ell)$, the mixing parameter $\bar{\chi}$, the $\mathrm{b}$ fragmentation, and sometimes the background fractions are 


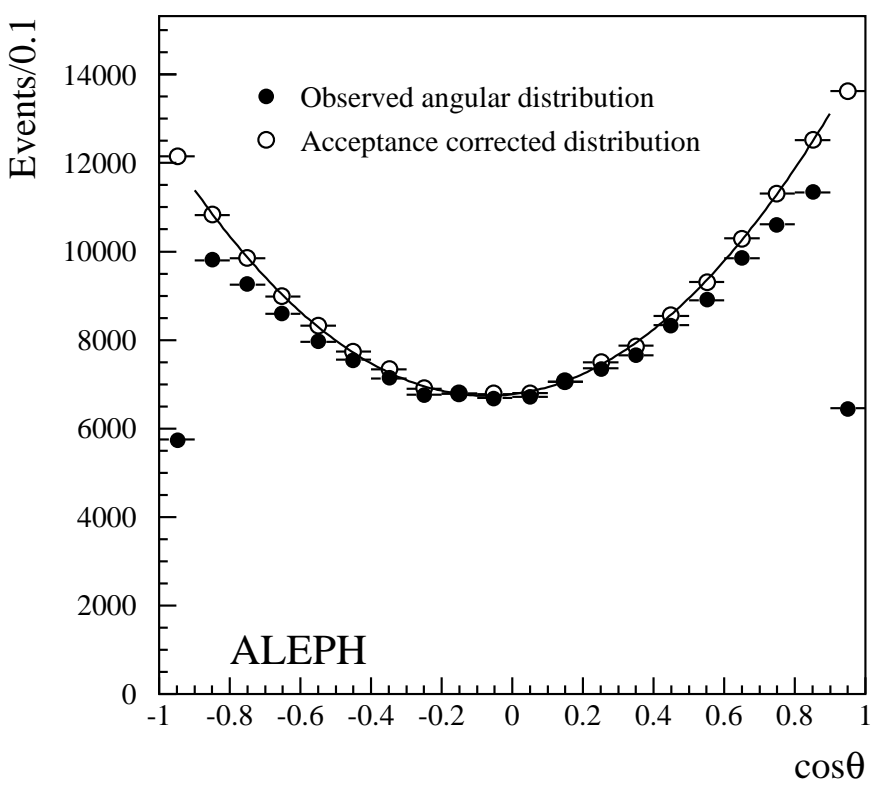

Figure 13: Observed (closed points) and acceptance corrected (open points), signed $\cos \theta_{\text {thrust }}$ distribution from the ALEPH lepton analysis.

\begin{tabular}{|l|c|c|}
\hline Lepton source & charge correlation & fraction for $p_{t}>1.25 \mathrm{GeV}$ \\
\hline $\mathrm{b} \rightarrow \ell^{-}, \mathrm{b} \rightarrow \overline{\mathrm{c}} \rightarrow \ell^{-}$ & 1 & 0.795 \\
$\mathrm{~b} \rightarrow \mathrm{c} \rightarrow \ell^{+}$ & -1 & 0.046 \\
$\mathrm{c} \rightarrow \ell^{+}$ & 1 & 0.048 \\
background & weak & 0.111 \\
\hline
\end{tabular}

Table 5: Correlation between the lepton charge and the quark charge at decay time. Also shown is the sample composition for $p_{t}>1.25 \mathrm{GeV}$ in the ALEPH analysis.

measured in the same or similar analyses as explained in section 5.4. If $\bar{\chi}$ is measured with the same experimental cuts as $A_{\mathrm{FB}}^{\mathrm{b}}$, its measurement can be seen as a measurement of the charge tagging efficiency. Then all systematic uncertainties in $A_{\mathrm{FB}}^{\mathrm{b}}$ due to the ratios of the different b sources cancel completely and only uncertainties from non-b background remain. Also here large cancellations occur, since the branching ratios and fragmentation parameters act as an effective parametrisation of the lepton spectra in $b \bar{b}$ and $c \bar{c}$ events.

\subsubsection{D-meson measurements}

Like that of the lepton, the charge of a D-meson is correlated to that of the quark. However, apart from the suppressed $\mathrm{b} \rightarrow \overline{\mathrm{c}}$ decays, there is only one channel per flavour and for b's the correlation is opposite to that in the lepton case. The opposite correlation reduces the error significantly when lepton and D measurements are combined.

Due to the better reconstruction efficiency and lower background at high energies, the Dmeson analyses are, again complementary to the leptons, more sensitive to $A_{\mathrm{FB}}^{\mathrm{c}}$ than to $A_{\mathrm{FB}}^{\mathrm{b}}$. DELPHI [52] and OPAL [55] measure $A_{\mathrm{FB}}^{\mathrm{c}}$ and $A_{\mathrm{FB}}^{\mathrm{b}}$ with a soft cut on the D-momentum. $A_{\mathrm{FB}}^{\mathrm{b}}$ is included mainly to treat the correlations correctly. ALEPH [56] measures $A_{\mathrm{FB}}^{\mathrm{c}}$ only, applying 
a harder D-momentum cut.

Since the total reconstruction efficiency cancels in the asymmetry measurement, many channels can be used. The background is well under control using the mass sidebands. For the $\mathrm{D}^{*+}$ channels, the charge is uniquely given by the slow pion from the $\mathrm{D}^{*+} \rightarrow \mathrm{D}^{0}$ decay, so that reflections from unwanted $\mathrm{D}^{0}$ decay modes are also not disturbing.

\subsubsection{Jetcharge measurements}

It was already seen at PEP [57] that the charge of the hadrons in a jet contains information about the charge of the originating quark. All LEP experiments [52,58-60] and SLD [61] have used this technique to measure the b-asymmetry. Usually the jetcharge is defined as

$$
Q_{h}=\frac{\sum_{i} q_{i} p_{\| i}^{\kappa}}{\sum_{i} p_{\| i}^{\kappa}},
$$

where the sum runs over all charged particles in a hemisphere with charge $q_{i}$ and longitudinal momentum $p_{\| i}$, and $\kappa$ is a tunable parameter with typical values between 0.3 and 1 .

With $Q_{\mathrm{F} / \mathrm{B}}$ being the jetcharge of the forward/backward hemisphere and $Q_{\mathrm{f} / \overline{\mathrm{f}}}$ the jetcharge of the quark/antiquark hemisphere, one has

$$
\begin{aligned}
\left\langle Q_{\mathrm{FB}}\right\rangle & =\delta_{\mathrm{f}} A_{\mathrm{FB}}^{\mathrm{f}} \\
\left\langle Q_{\mathrm{FB}}\right\rangle & =\left\langle Q_{\mathrm{F}}-Q_{\mathrm{B}}\right\rangle \\
\delta_{\mathrm{f}} & =\left\langle Q_{\mathrm{f}}-Q_{\overline{\mathrm{f}}}\right\rangle
\end{aligned}
$$

for a pure sample of $\mathrm{f} \overline{\mathrm{f}}$-events. The resolution parameter $\delta_{\mathrm{f}}$ can be measured from data, taking only some corrections due to hemisphere correlations from the simulation. These correlations arise from effects like charge conservation, hard gluon radiation etc.

It should be noted that dilution due to $\mathrm{B}^{0} \overline{\mathrm{B}^{0}}$-mixing is completely absorbed into the measured $\delta_{\mathrm{b}}$. Effects from gluon radiation are also included to a large extent, so that the residual QCD correction is very small.

In reality two additional complications arise. The selected sample is not $100 \%$ pure and the acceptance is not constant in $\cos \theta$. Background corrections are typically taken from the simulation whereas polar angle corrections are evaluated from the measured polar angle variation of the tagging rate and some Monte Carlo corrections.

\subsubsection{Other measurements}

In addition to the methods described above, vertex charge and charged Kaons have been used to measure asymmetries.

In principle the decay chains $\mathrm{b} \rightarrow \mathrm{c} \rightarrow \mathrm{s}$ and $\mathrm{c} \rightarrow \mathrm{s}$ give a correlation between the Kaon charge and the b- or c-quark charge. In practice, however, there exists also background from fragmentation and from additional Kaons in B- and D-decays. SLD have measured $\mathcal{A}_{\mathrm{b}}$ using $\mathrm{K}^{ \pm}[62,63]$. Light quark background is rejected by their lifetime/mass b-tag and fragmentation background is suppressed by requiring the $\mathrm{K}^{ \pm}$to be consistent with coming from the secondary vertex. This measurement is currently limited by the systematics from the Kaon content in B-decays which has been taken from lower energy data, but with the new SLD vertex detector the efficiency should be high enough to measure the charge tagging efficiency from the data by comparing same sign with opposite sign double tags. 
Another way to tag the quark charge is to measure the charge of all particles associated to a secondary vertex. The efficiency for measuring the vertex charge correctly is given roughly by $\epsilon^{n}$, where $\epsilon$ is the efficiency for associating a track uniquely to a vertex and $n$ is the charged multiplicity of the decay. OPAL has combined a vertex charge technique with a jetcharge technique to measure $A_{\mathrm{FB}}^{\mathrm{b}}[60]$. However, due to the high B-decay multiplicity and the relatively low single track tagging efficiency at LEP, the efficiency for measuring a vertex charge is only about $1 \%$ and the accuracy on $A_{\mathrm{FB}}^{\mathrm{b}}$ is completely dominated by the jetcharge analysis.

SLD has performed the most precise measurement of $\mathcal{A}_{\mathrm{c}}$ using a combination of charged Kaons and vertex charge $[63,64]$. The powerful lifetime/mass tag at SLD allows a clean charm sample to be separated, and due to the better single track efficiency at SLD and the smaller decay multiplicity for charm, the vertex charge efficiency is about ten times larger than for the OPAL b-analysis.

\subsubsection{QCD corrections to asymmetries}

The QCD corrections to the asymmetries explained in section 2.2 were calculated assuming a perfect detector with full acceptance. The analyses, however, always apply some cuts changing the composition of the sample. For the D-meson and lepton analyses the main effect is due to momentum cuts. The largest part of the QCD correction is coming from events where a high momentum gluon is radiated and a low momentum quark consequently has a direction far from its original one. The momentum cuts on the lepton or D-meson reduce the fraction of such events significantly, reducing also the QCD correction to be applied to the result. As an example figure 14 shows the QCD correction as a function of the $\mathrm{D}^{*+}$ momentum for the OPAL analysis. It can be seen that for high $\mathrm{D}^{*+}$ momenta the correction vanishes completely.

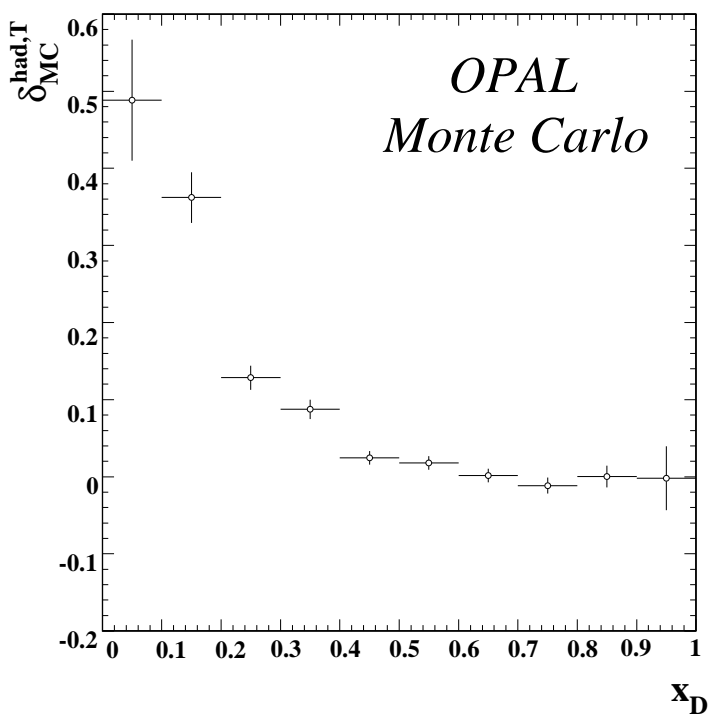

Figure 14: Experimental bias on the QCD correction to $A_{\mathrm{FB}}^{\mathrm{c}}$ as a function of the $\mathrm{D}^{*+}$ momentum.

For the jetcharge measurements the situation is by far more complicated. Since strong gluon radiation reduces simultaneously the asymmetry and the charge separation, which is measured from the data themselves, the QCD corrections are largely accounted for intrinsically. However, since gluon radiation introduces additional hemisphere correlations and since these 
effects depend on the polar angle, it is difficult to obtain a number for the residual QCD correction. It is however possible to take these effects into account in a global fragmentation plus detector correction, so that the results obtained with this method are reliable.

The procedure applied by the LEP experiments is as follows. In general the QCD correction can be written as $A_{\mathrm{FB}}^{\mathrm{q}}=\left(1-C_{\mathrm{q}}\right)\left(A_{\mathrm{FB}}^{\mathrm{q}}\right)_{\text {no QCD }}$. The total QCD corrections in second order using the thrust axis as reference and including fragmentation effects have been estimated to be $C_{\mathrm{b}}=(2.96 \pm 0.40) \%$ and $C_{\mathrm{c}}=(3.57 \pm 0.76) \%$ [65]. These corrections are reasonably well reproduced by event generators like JETSET and HERWIG. For each analysis the simulation is used to estimate which fraction of the full correction has to be applied, and the total correction is scaled accordingly. The $C_{\mathrm{q}}$ are typically reduced by about a factor of two, but with a large spread between the different analyses. All asymmetry results quoted are fully corrected for QCD effects.

\subsubsection{Asymmetry Results}

All LEP results on $A_{\mathrm{FB}}^{\mathrm{b}}$ and $A_{\mathrm{FB}}^{\mathrm{c}}$ are summarised in figure 15 . $A_{\mathrm{FB}}^{\mathrm{b}}$ measurements using Dmesons have much larger errors than the ones with other techniques and are not included in figure 15. Good agreement between the different experiments and methods can be observed.

Figure 16 summarises the SLD results on $\mathcal{A}_{\mathrm{b}}$ and $\mathcal{A}_{\mathrm{c}}$ from the measurements of the leftright-forward-backward asymmetries. Also here the results of the different methods agree well.

\section{$5.2 \quad R_{\mathrm{b}}$ measurements}

All accurate measurements of $R_{\mathrm{b}}$ are primarily single/double tag comparisons. If $f_{s}$ denotes the fraction of b-tagged hemispheres and $f_{d}$ the fraction of events where both hemispheres are tagged one has:

$$
\begin{aligned}
& f_{s}=\varepsilon_{\mathrm{b}} R_{\mathrm{b}}+\varepsilon_{\mathrm{c}} R_{\mathrm{c}}+\varepsilon_{\mathrm{uds}}\left(1-R_{\mathrm{b}}-R_{\mathrm{c}}\right) \\
& f_{d}=\varepsilon_{\mathrm{b}}^{(d)} R_{\mathrm{b}}+\varepsilon_{\mathrm{c}}^{(d)} R_{\mathrm{c}}+\varepsilon_{\mathrm{uds}}^{(d)}\left(1-R_{\mathrm{b}}-R_{\mathrm{c}}\right),
\end{aligned}
$$

where $\varepsilon_{\mathrm{f}}$ is the hemisphere tagging efficiency for flavour $\mathrm{f}$. The double tagging efficiency $\varepsilon_{\mathrm{f}}^{(d)}$ can be written as $\varepsilon_{\mathrm{f}}^{(d)}=\left(1+\mathcal{C}_{\mathrm{f}}\right) \varepsilon_{\mathrm{f}}^{2}$ where the factor $\mathcal{C}_{\mathrm{f}} \neq 0$ corrects for the fact that the two hemispheres in an event are slightly correlated. For the pure b-tags, however, $\mathcal{C}_{\mathrm{c}}$ and $\mathcal{C}_{\text {uds }}$ can be safely neglected.

Neglecting hemisphere correlations and background, $R_{\mathrm{b}}$ can be obtained from $R_{\mathrm{b}}=f_{s}^{2} / f_{d}$ so that the knowledge of the badly known b-tagging efficiency $\varepsilon_{\mathrm{b}}$ is not needed. However, the background efficiencies $\varepsilon_{\mathrm{c}}, \varepsilon_{\mathrm{uds}}$ and the hemisphere correlations $\mathcal{C}_{\mathrm{b}}$ have to be taken from Monte Carlo. The uncertainties on these parameters are included in the systematic errors. The effect of an uncertainty $\Delta \varepsilon_{x}$ from a background source $x$ is approximately given by $\Delta R_{\mathrm{b}}=2 \frac{\Delta \varepsilon_{x}}{\varepsilon_{\mathrm{b}}} R_{x}$ and for an uncertainty on the correlation by $\Delta R_{\mathrm{b}}=\Delta \mathcal{C}_{\mathrm{b}} R_{\mathrm{b}}$.

\subsubsection{Light quark background}

With the modern tagging techniques, light quark events are typically suppressed by more than a factor 100 with respect to b-events. Using tracks with negative impact parameters or vertices with negative decay length, the detector efficiency and resolution is normally well under control. $\mathrm{K}_{\mathrm{S}}^{0}$ and $\Lambda$ decays are normally far enough away from the primary vertex that their 

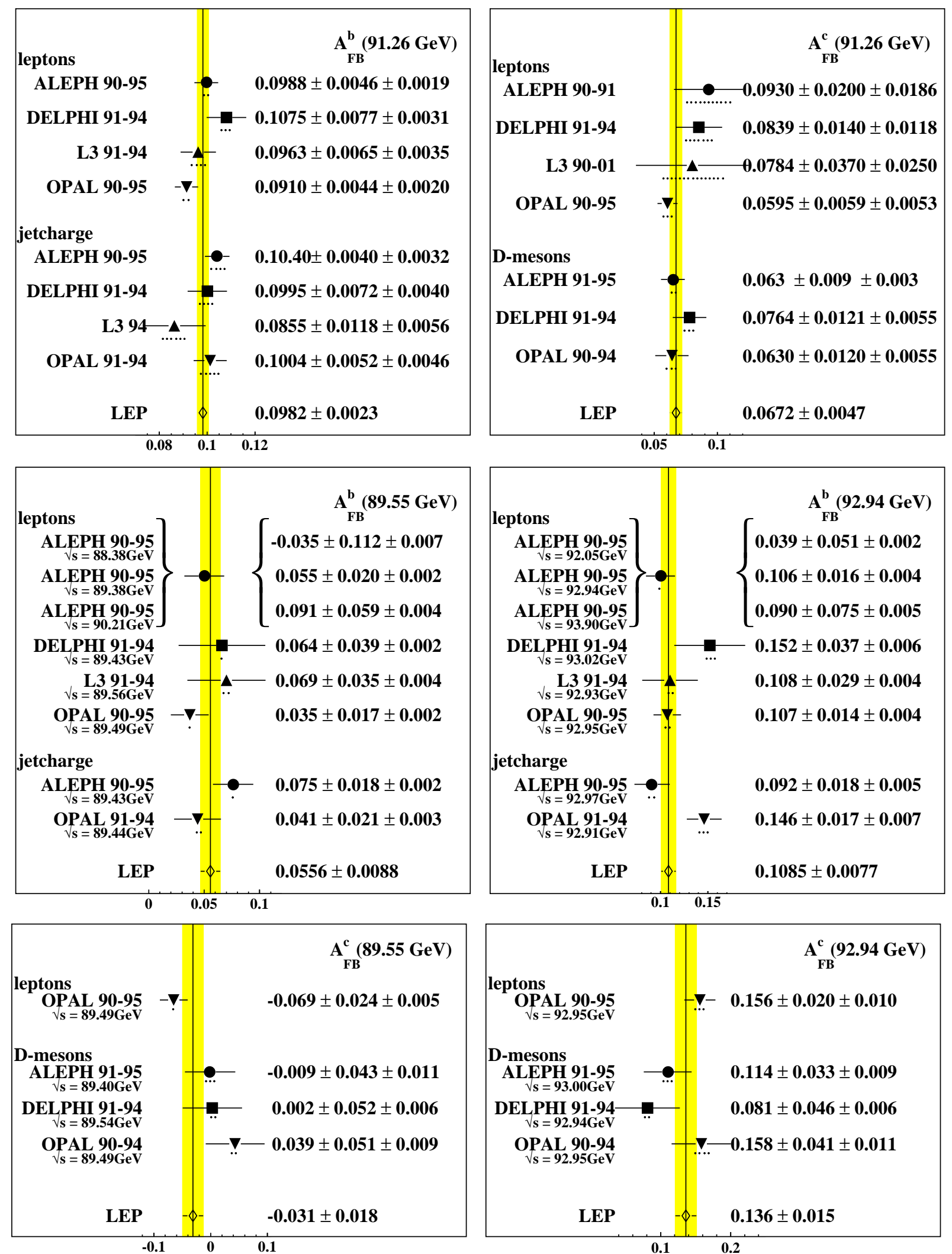

Figure 15: b- and c-quark forward-backward asymmetries measured at LEP at different centre of mass energies. The printed numbers are taken from the experimental notes. The points with the error bars correspond to the results with the agreed input parameters. The dotted error bars represent the systematic uncertainties. 

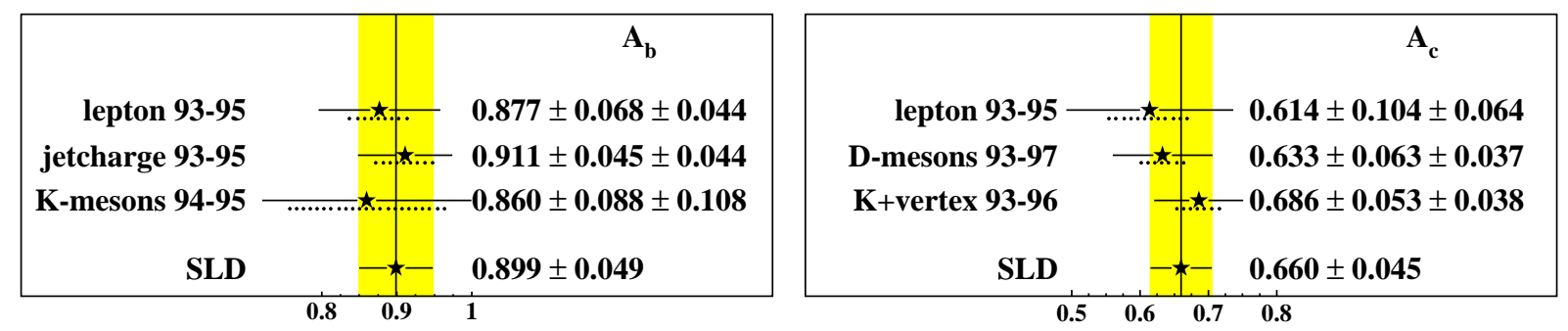

Figure 16: $\mathcal{A}_{\mathrm{b}}$ and $\mathcal{A}_{\mathrm{c}}$ measurements by SLD.

decay products are not included in the b-tagging. In addition they are searched for by special pairing algorithms so that the few remaining ones can be removed.

The most serious source of background in a light quark event is thus the splitting of a gluon into a heavy quark pair $g \rightarrow c \bar{c}$ and especially $g \rightarrow b \bar{b}$. The probability that an event contains gluon splitting to $\mathrm{c} \overline{\mathrm{c}}$ has been measured by OPAL to be $P(g \rightarrow \mathrm{c} \overline{\mathrm{c}})=(2.38 \pm 0.4) \cdot 10^{-2}$ [66], which is used by all experiments. For the gluon splitting to $\mathrm{b} \overline{\mathrm{b}}$, the theoretical expectation $\frac{P(g \rightarrow \mathrm{b} \overline{\mathrm{b}})}{P(g \rightarrow \mathrm{c})}=0.13 \pm 0.04$ [67] is used which is, however, in agreement with measurements by ALEPH [68] and DELPHI [69].

Since the heavy hadrons from gluon splitting are normally much less energetic than the ones from primary quark fragmentation, the tagging efficiencies for gluon splitting events are

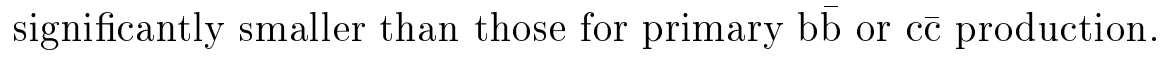

\subsection{2 c-quark background}

Since c-hadrons have lifetimes comparable to b-hadrons, the estimate of $\varepsilon_{\mathrm{c}}$ is more difficult than that of $\varepsilon_{\mathrm{uds}}$. Three major complications arise for the charm sector:

- Due to lifetime differences of up to a factor of four, the tagging efficiencies vary strongly between the different charmed hadrons. Since the $\mathrm{D}^{*+}$ can decay into $\mathrm{D}^{0} \pi^{+}$and $\mathrm{D}^{+}\left(\pi^{0}\right.$ or $\left.\gamma\right)$, but the $\mathrm{D}^{* 0}$ only into $\mathrm{D}^{0} \gamma$, not even the $\mathrm{D}^{+} / \mathrm{D}^{0}$ ratio is trivial to predict.

- A full set of D branching ratio measurements does not exist. For the more inclusive branching ratios which are relevant for the estimate of $\varepsilon_{\mathrm{c}}$, only relatively old measurements at the $\psi^{\prime \prime}$ are available.

- Around the working point, $\varepsilon_{\mathrm{c}}$ varies quite rapidly as a function of the b-tagging cut so that $\varepsilon_{\mathrm{c}}$ depends strongly on the understanding of detector properties.

The production ratios of the different weakly decaying charmed hadrons are now measured at LEP with good accuracy. They are obtained together with $R_{\mathrm{c}}$ and are therefore described in more detail in section 5.3.3.

The decay properties of the charmed hadrons that affect mostly the determination of $\varepsilon_{\mathrm{c}}$ are the charged decay multiplicities and the branching ratios for the decay $\mathrm{D} \rightarrow \mathrm{K}^{0} X$. The decay multiplicities are known on the few percent level and are taken from an analysis from Mark III [70]. In a $\mathrm{D} \rightarrow \mathrm{K}^{0} X$ decay the $\mathrm{K}^{0}$ carries a lot of energy and invariant mass, so that a hemisphere with this decay is normally not tagged. The branching ratios are taken from the PDG [6] and are known with about $5 \%$ precision. 
Charmed hadron lifetimes are known with good precision from fixed target experiments [6], so they contribute only weakly to the systematic uncertainty.

Charm fragmentation is also of minor importance. The value for the mean charmed hadron energy is taken from LEP [71].

All $R_{\mathrm{b}}$ measurements need explicit assumptions on the value of $R_{\mathrm{c}}$ to determine the charm background. This leads to a correlation of $-15 \%$ between $R_{\mathrm{b}}$ and $R_{\mathrm{c}}$.

\subsubsection{Hemisphere correlations}

The second major source of systematic uncertainties is the estimate of the hemisphere correlations. The main sources for those are:

- angular effects: The b-tagging efficiency depends significantly on the polar angle of a jet. At smaller polar angles the resolution of the vertex detector gets worse due to larger multiple scattering. In addition, close to the edges of the detector more tracks are lost outside its acceptance. Usually the two jets in an event are back to back, so that both jets are either in regions of good or bad efficiency. There is also some dependence on the azimuthal angle due to the flatness of the beamspot and due to inhomogeneities of the detector itself.

- gluon radiation: There are two effects from gluon radiation that lead to sizeable hemisphere correlations. Firstly, in about $2 \%$ of the events the two b's are boosted into the same hemisphere, so that one side contains only a high energy gluon, leading to a negative correlation. Secondly, the b-tagging efficiency is a function of the energy of the b-hadron. A gluon radiated at large angle takes energy from both quarks, reducing the energy on both sides and leading to a positive correlation. If the efficiency-momentum dependence is linear, the size of the latter effect is not altered by the emission of additional collinear gluons or by fragmentation [72], so that it can be estimated in perturbative QCD. Calculations by Nason and Oleari estimated effects of about 1\% [73,74] consistent with fragmentation models [9]. In practice the dependence is somewhat weaker than linear, so that the resulting effect is smaller.

- vertex bias: Some of the older analyses use a common primary vertex for both hemispheres. If tracks from a b-hadron decay are included in the primary vertex, this can influence the b-tagging on both sides. The size of this effect is difficult to estimate but it depends on the details of the b-fragmentation and decay. For that reason the more recent analyses calculate the primary vertex separately for each hemisphere, even if this results in a slightly smaller efficiency at LEP. The beamspot, although common to both hemispheres, can safely be included in the vertex fit. At SLD it is much smaller than the detector resolution and at LEP it is significantly smaller in the vertical and significantly larger in the horizontal direction, so that it never has a significant effect on the correlation.

Since the different sources are correlated with each other, the final $\mathcal{C}_{\mathrm{b}}$ to correct $R_{\mathrm{b}}$ is usually calculated with the simulation from the ratio of the double tag efficiency and the square of the

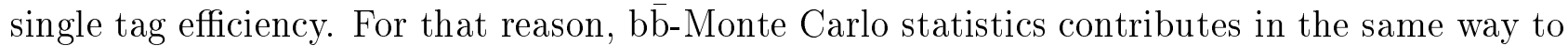
the total uncertainty as the data.

The breakdown of the correlations into different sources is, however, needed to assess the systematic uncertainties. For most correlation sources a test quantity can be defined, and the 
tagging efficiency can be measured in data and Monte Carlo as a function of this variable, where background corrections are taken from simulation. As an example, for the polar angle dependence in the approximation that the events are back-to-back, $z=\cos \theta$ of the thrust axis can be used and the efficiency as a function of this variable can be studied. The correlation due to the polar angle $\mathcal{C}_{\theta}$ then can be calculated as

$$
\mathcal{C}_{\theta}=\frac{2 \int_{0}^{z_{\max }} d z f(z) \varepsilon_{\mathrm{b}}(z) \cdot \varepsilon_{\mathrm{b}}(-z)}{\left(\int_{-1}^{1} d z f(z) \varepsilon_{\mathrm{b}}(z)\right)^{2}} .
$$

The difference between $\mathcal{C}_{\theta}$ in data and simulation is used as an estimate of the accuracy of the Monte Carlo prediction.

\subsubsection{Multitag extensions}

ALEPH and DELPHI have extended their $R_{\mathrm{b}}$ measurements using a multitag technique. For this technique several, mutually exclusive, tags are used that are sensitive to different flavours. ALEPH and DELPHI both use five tags: three b-tags, one charm tag and one uds-tag. With these tags they measure all five single and fifteen double tag rates. Taking the charm and uds efficiency for the primary b-tag and all hemisphere correlations from the simulation, $R_{\mathrm{b}}$ and the other tagging efficiencies can be measured from the data. For that reason all but the first b-tag can be fairly complicated algorithms since a detailed understanding of their efficiencies is not needed.

The multitag extension improves the statistical precision of an $R_{\mathrm{b}}$ analysis by about $10-$ $20 \%$ compared to a single/double tag technique with the primary tag only. The sensitivity to the light quark efficiencies however remains unchanged. The systematic uncertainty from background sources can also be reduced slightly, since the better statistical power allows a harder cut in the primary tag.

The sensitivity to the hemisphere correlation of the primary b-tag is reduced by a factor $0.5-0.8$. This gain is however mostly compensated by the sensitivity to the other correlation coefficients, mainly between the primary and the secondary b-tags.

\subsection{5 $R_{\mathrm{b}}$ results}

The results of the $R_{\mathrm{b}}^{0}$ measurements at LEP and SLC [42-45,63,75] with $R_{\mathrm{c}}$ fixed to its Standard Model expectation $\left(R_{\mathrm{c}}=0.172\right)$ are shown in figure 17. Also shown are an older measurement by L3 with a neural network tagging using event shapes [76] and the average of the lepton measurements performed at LEP (section 5.4). The average has been obtained by a global fit to all data presented in section 5.6. The error breakdown of the five lifetime tag measurements is shown in table 6 .

For the most accurate measurements the largest single source of systematic error is now the probability of a gluon to split into a b $\bar{b}$ pair. Since these events contain true b-quarks this background is irreducible and the error can only be decreased by a better understanding of the gluon splitting process.

Since the uncertainties of all measurements contain a substantial fraction common with the other experiments, the agreement of the analyses cannot be judged easily from figure 17 . For that reason the five accurate single/double tag measurements only were combined with the procedure described in section 5.5 with $R_{\mathrm{c}}$ fixed to its Standard Model expectation. The result of the combination was $R_{\mathrm{b}}^{0}=0.21669 \pm 0.00093$ with $\chi^{2} / n d f=1.5 / 4$, indicating good agreement between the different results. 


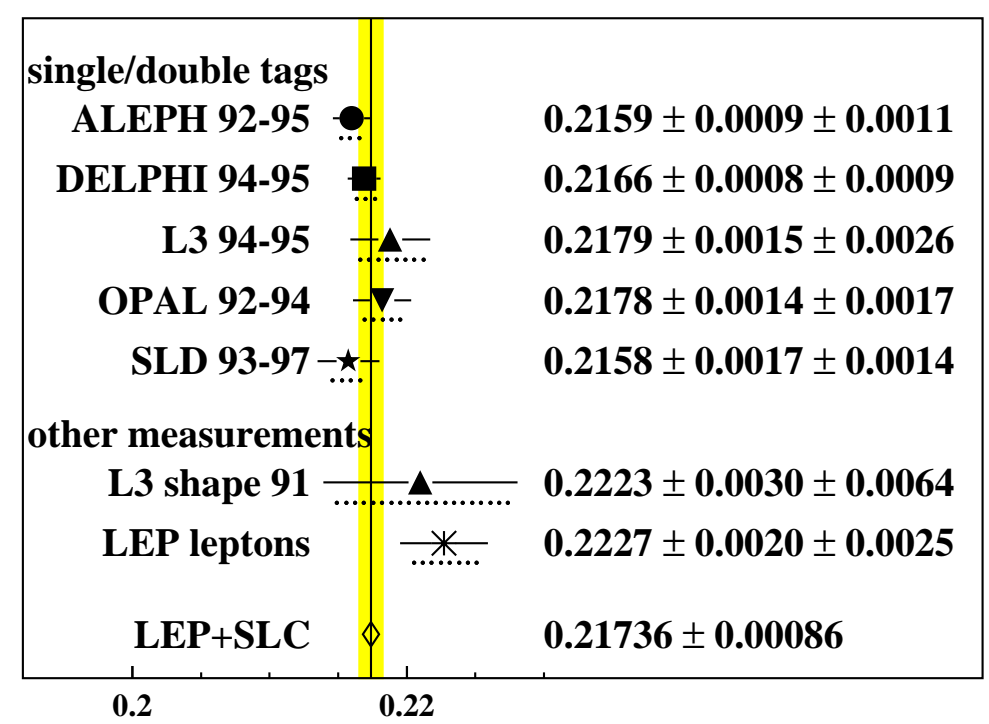

Figure 17: Measurements of $R_{\mathrm{b}}^{0}$ at LEP and SLC with $R_{\mathrm{c}}$ fixed to its Standard Model expectation $\left(R_{\mathrm{c}}=0.172\right)$. The notation is as in figure 15 .

\begin{tabular}{|l|c|c|c|c|c|}
\hline \multirow{2}{*}{} & \multicolumn{2}{|c|}{ multitag } & \multicolumn{3}{c|}{ single/double tag } \\
\cline { 2 - 6 } & ALEPH & DELPHI & L3 & OPAL & SLD \\
\hline statistics & 0.0009 & 0.0008 & 0.0015 & 0.0014 & 0.0017 \\
\hline MC statistics & 0.0005 & 0.0005 & 0.0008 & 0.0003 & 0.0003 \\
event selection & 0.0001 & 0.0001 & & 0.0003 & 0.0003 \\
detector resolution & 0.0005 & 0.0002 & 0.0004 & 0.0004 & 0.0010 \\
$\varepsilon_{\text {b correlation }}$ & 0.0003 & 0.0004 & 0.0007 & 0.0010 & 0.0002 \\
\hline udsc simulation & 0.0005 & 0.0005 & 0.0018 & 0.0009 & 0.0003 \\
gluon splitting & 0.0006 & 0.0005 & 0.0002 & 0.0006 & 0.0005 \\
\hline total systematic & 0.0011 & 0.0009 & 0.0026 & 0.0017 & 0.0014 \\
\hline
\end{tabular}

Table 6: Error breakdown of the lifetime tag $R_{\mathrm{b}}$ analyses.

DELPHI has also measured the variation of $R_{\mathrm{b}}$ with the centre of mass energy for the energy-scans in 1993 and 1995. Since the b-tagging efficiency varies very little for the energies used in the scan, the ratios of the off-peak to the on-peak $R_{\mathrm{b}}$ can be obtained from the ratio of tagging rates at the two energies. No single/double tag comparison is needed, so that the more powerful event tag can be used. Also the uncertainties due to backgrounds cancel to a large extent in the ratio, shifting the optimal working point towards higher efficiencies. The result obtained by DELPHI [42] was:

$$
\begin{aligned}
& \frac{R_{\mathrm{b}}(89.46 \mathrm{GeV})}{R_{\mathrm{b}}(91.27 \mathrm{GeV})}=0.9852 \pm 0.0091 \\
& \frac{R_{\mathrm{b}}(93.00 \mathrm{GeV})}{R_{\mathrm{b}}(91.27 \mathrm{GeV})}=1.0033 \pm 0.0082
\end{aligned}
$$

compared to 0.9964 (0.9984) predicted by the Standard Model for the lower (higher) energy.

If new physics appears only in loop corrections no variation on this prediction is expected. However this measurement can severely constrain new Born level physics like an R-parity 
violating sneutrino exchange [77].

\section{$5.3 \quad R_{c}$ measurements}

Mainly four types of measurements of $R_{\mathrm{c}}$ are performed at LEP/SLD:

- single/double tags,

- exclusive/inclusive single/double tags,

- charm counting,

- lepton measurements.

All methods have about equal weight in the final result. Contrary to the $R_{\mathrm{b}}$ analyses in the $R_{\mathrm{c}}$ analyses the $\mathrm{b} \overline{\mathrm{b}}$ background is normally evaluated directly from the tagged data sample using the different b-tagging tools, so that the $R_{\mathrm{c}}$ results mostly have no dependence on an assumed value of $R_{\mathrm{b}}$.

\subsubsection{Single/double tags}

The principle of the single/double tag technique is the same for $R_{\mathrm{c}}$ as for $R_{\mathrm{b}}$. Different tagging methods, however, are used to tag charm.

Because of the small beampipe and the pointlike beamspot SLD $[63,78]$ is able to apply a tag using lifetime and vertex mass. For secondary vertices with masses between 0.6 and $2 \mathrm{GeV}$ the two dimensional cut in the vertex-mass/momentum plane explained in figure 8 is applied resulting in a charm tagging efficiency of $14 \%$ with a purity of $67 \%$. The background is mostly due to b $\bar{b}$ events. The b-tagging efficiency of the charm tag can be measured accurately using hemispheres opposite to a b-tagged one, so that it does not give rise to a significant uncertainty. This is the by far most accurate method. However, due to the lower statistics of SLD up to now, it gives about the same total error as the LEP results.

At LEP ALEPH [79] has done a single/double tag measurement using fully reconstructed D-mesons. They obtain $2.5 \%$ efficiency and $89 \%$ purity. Due to the low tagging efficiency the method is severely limited by the statistics of the double tag sample.

DELPHI [80] has tried a single/double tag measurement using low $p_{t}$ pions. However this measurement is strongly limited by the understanding of the background in the single tag sample.

\subsubsection{Exclusive/inclusive single/double tags}

In a single/double tag analysis, the systematic error is determined by the purity of the single tag sample, while the statistical error is due to the number the double tagged events.

To overcome both problems simultaneously, ALEPH [79], DELPHI [80] and OPAL [49] use an exclusive/inclusive single/double tag technique. In a first step, the rate of $\mathrm{c} \rightarrow \mathrm{D}^{*+} \rightarrow \pi^{+} \mathrm{D}^{0}$ events is measured using a $\mathrm{D}^{0}$-decay mode with well known branching ratio, typically $\mathrm{D}^{0} \rightarrow$ $\mathrm{K}^{-} \pi^{+}$. This rate is proportional to $R_{\mathrm{c}} \times \mathrm{P}\left(\mathrm{c} \rightarrow \mathrm{D}^{*+}\right) \times \mathrm{BR}\left(\mathrm{D}^{*+} \rightarrow \pi^{+} \mathrm{D}^{0}\right)$. In a second step, charm is tagged with an exclusively reconstructed high energy $\mathrm{D}^{*+}$ in one hemisphere and the $\mathrm{D}^{*+}$ rate is measured in the opposite hemisphere with low $p_{t}$ pions. This rate is proportional to $\mathrm{P}\left(\mathrm{c} \rightarrow \mathrm{D}^{*+}\right) \times \mathrm{BR}\left(\mathrm{D}^{*+} \rightarrow \pi^{+} \mathrm{D}^{0}\right)$, so that the ratio of the two rates gives $R_{\mathrm{c}}$. 
In this method the uncertainty in the charm tagging efficiency due to fragmentation effects mainly cancels. However the reconstruction efficiency for the $\mathrm{D}^{0} \rightarrow \mathrm{K}^{-} \pi^{+}$decay and the efficiency with which the slow pion rate can be extracted from the $p_{t}$ spectrum, still need to be known from simulation.

\subsubsection{Charm counting}

At LEP, $\mathrm{D}^{0}, \mathrm{D}^{+}, \mathrm{D}_{\mathrm{s}}$ mesons and $\Lambda_{\mathrm{c}}$ baryons can be reconstructed in decay modes with well known branching ratios (see figure 10). The production rate for a single charmed hadron $\mathrm{D}$ ( $\left.N_{\mathrm{D}} / N_{\text {event }}\right)$ is proportional to $R_{\mathrm{c}} f(\mathrm{D})$, where the probability that a c-quark ends up in a specific D-hadron state $(f(D))$ is a priory unknown. However, after fragmentation and resonance decays all charm quarks end up in a weakly decaying charmed hadron, so that the production probabilities for these states add up to one. In principle $R_{\mathrm{c}}$ can thus be measured from the sum of the production rates of all weakly decaying charmed hadrons.

Charmed strange baryons have, however, up to now not been measured. Extrapolating from the light quark sector, their rate is estimated to be $(15 \pm 5) \%$ times the $\Lambda_{\mathrm{c}}$ rate, giving:

$$
f\left(\mathrm{D}^{0}\right)+f\left(\mathrm{D}^{+}\right)+f\left(\mathrm{D}_{\mathrm{s}}\right)+(1.15 \pm 0.05) f\left(\Lambda_{\mathrm{c}}\right)=1,
$$

so that $R_{\mathrm{c}}$ can be obtained from the four production rates measured at LEP. The error from the charmed strange baryon rate turns out to be small, and the only remaining uncertainty from fragmentation is from a small extrapolation to low charmed hadron energies.

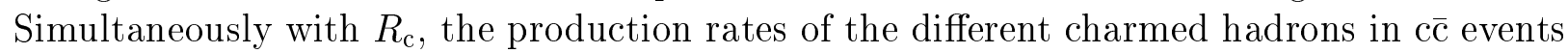
are obtained, these need to be known to estimate the cic background in the $R_{\mathrm{b}}$-analyses. ALEPH [47], DELPHI [80] and OPAL [81] have measured $R_{\mathrm{c}}$ using this technique.

\subsubsection{Lepton measurements}

ALEPH has also measured $R_{\mathrm{c}}$ from their electron spectra [79]. The total electron spectrum can be written as

$$
\mathcal{P}\left(p, p_{t}\right)=R_{\mathrm{b}} \mathcal{P}_{\mathrm{b}}\left(p, p_{t}\right)+R_{\mathrm{c}} \mathcal{P}_{\mathrm{c}}\left(p, p_{t}\right)+\left(1-R_{\mathrm{b}}-R_{\mathrm{c}}\right) \mathcal{P}_{\mathrm{uds}}\left(p, p_{t}\right)
$$

$\mathcal{P}_{\mathrm{b}}$ can be measured without model dependence using hemispheres opposite to a b-tagged one, and $\mathcal{P}_{\text {uds }}$ can be obtained from reconstructed photon conversions and samples of identified hadrons.

$\mathcal{P}_{\mathrm{c}}$ can be written as $\mathcal{P}_{\mathrm{c}}\left(p, p_{t}\right)=\mathrm{BR}(\mathrm{c} \rightarrow \ell) P_{\mathrm{c}}\left(p, p_{t}\right)$ where the normalised spectra $P_{\mathrm{c}}\left(p, p_{t}\right)$ are obtained from a fit to lower energy data [82]. The branching ratio $\mathrm{BR}(\mathrm{c} \rightarrow \ell)$ is measured at ARGUS, PEP and PETRA to be $(9.8 \pm 0.5) \%$ [82]. Since the semileptonic branching ratios vary between the different charmed hadrons, this relies, however, on the assumption that the mixture of c-hadrons is independent of the centre of mass energies, which is suggested by a parton shower picture of fragmentation where the energy scale at which the fragmentation occurs is independent of the scale of the primary interaction.

As a cross check, DELPHI has measured BR $(\mathrm{c} \rightarrow \ell)$ from leptons opposite to a high energy $\mathrm{D}^{* \pm}$ to be $\mathrm{BR}(\mathrm{c} \rightarrow \ell)=(9.7 \pm 0.8 \pm 0.4) \%$ [83] , consistent with the number used. If one assumes that the different D-mesons have equal semileptonic partial width, $\mathrm{BR}(\mathrm{c} \rightarrow \ell)$ can also be obtained from the individual c-hadron semileptonic branching ratios and lifetimes $[6,84]$ and the D-production rates measured in the charm counting analyses. The result $(\mathrm{BR}(\mathrm{c} \rightarrow \ell)=$ $8.93 \pm 0.42 \%)$ is slightly lower but still in agreement with the assumed value. Due to its 
complicated correlation with the charm counting analysis it is, however, not possible to use this number directly in the analyses.

$R_{\mathrm{c}}$ is obtained from a fit to the electron spectrum with $R_{\mathrm{c}}$ and $R_{\mathrm{b}}$ as free parameters. In addition, ALEPH and DELPHI also measure $R_{\mathrm{c}}$ as a free parameter in their lepton fits described in section 5.4.

\subsection{5 $\quad R_{\text {c }}$ results}

Figure 18 summarises the $R_{\mathrm{c}}$ results at LEP and SLD. The average is again from the global fit to all data taking common systematics into account. To judge the agreement, the $R_{\mathrm{c}}$ measurements have been combined with the procedure described in section 5.5 leaving, apart from $R_{\mathrm{c}}$, the $\mathrm{D}^{*}$ and weakly decaying charmed hadron production probabilities as free parameters. The $\chi^{2}$ of this fit was 5.1 for 11 degrees of freedom indicating good agreement.

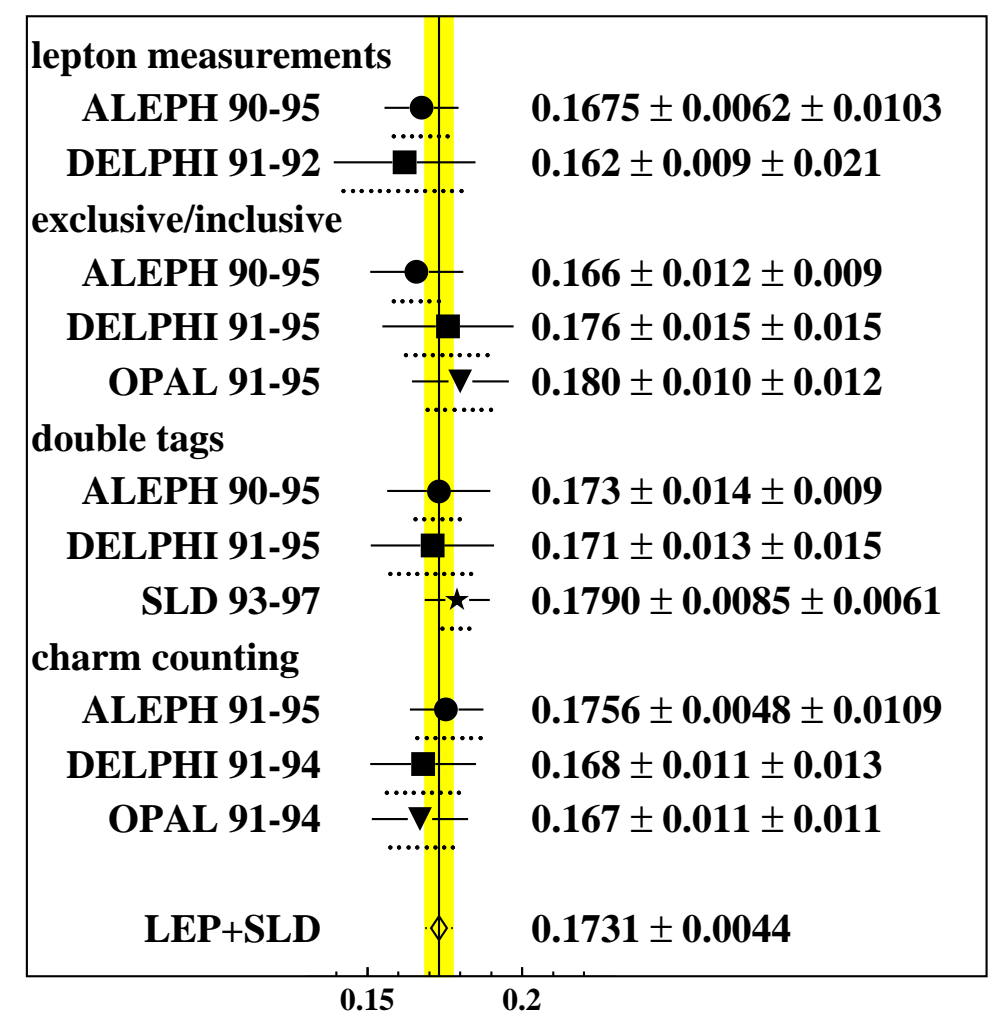

Figure 18: Measurements of $R_{\mathrm{c}}^{0}$ at LEP and SLC. The notation is as in figure 15.

\subsection{Lepton fits}

To measure the prompt $(\mathrm{b} \rightarrow \ell)$ and cascade $(\mathrm{b} \rightarrow \mathrm{c} \rightarrow \ell)$ b-semileptonic branching ratio and the average $\mathrm{b} \bar{b}$-mixing parameter, $(\bar{\chi})$ the experiments perform fits to their inclusive single and double lepton samples. In these fits, either the full single lepton sample is used [85-88] or b-tagging in the hemisphere opposite to the lepton is applied [89-91]. The second approach has the advantage that most of the background from misidentified hadrons can be suppressed, significantly reducing the systematic uncertainties. In the fits to the full spectra normally also $R_{\mathrm{b}}$ and sometimes $R_{\mathrm{c}}$ are left free. But compared to the lifetime tag analyses, these 
measurements are no longer competitive. In addition, parameters describing the hardness of the b- and c-quark fragmentation are often included in the fits. These parameters also absorb a significant part of the systematics from the modelling of the lepton spectra.

The main use of the $b \bar{b}$-mixing parameter, $\bar{\chi}$, is as a correction factor to the b-asymmetry. For that reason, it is often measured simultaneously with the asymmetries to profit from the error cancellation in an optimal way $[50,51,53]$.

The measurement of the semileptonic branching ratios and of the mixing parameter is clearly important for the understanding of B-hadron production and decay. However because of their relevance for the asymmetry measurements, as explained in section 5.1.1, and their simultaneous correlation with $R_{\mathrm{b}}$ and $R_{\mathrm{c}}$, they are included in the combination procedure of the electroweak observables.

\subsection{Combination of results}

All quantities are measured with several methods and apart from $\mathcal{A}_{\mathrm{b}}$ and $\mathcal{A}_{\mathrm{c}}$ by several collaborations. In addition some analyses of one electroweak parameter have to assume a value for another one, e.g. $R_{\mathrm{b}}$ needs the knowledge of $R_{\mathrm{c}}$ to correct for the charm background. For these reasons a well defined combination procedure is needed. To facilitate this task the LEP experiments and SLD have agreed on a common set of input parameters and their errors ${ }^{7}[71,82]$. This assures that the meanings of the common systematic errors are the same, so that reliable weights can be calculated.

As already seen from the description of the measurements, some parameters that are needed to correct one electroweak quantity are measured together with another one, as e.g. the charmed hadron fraction in $c \overline{\mathrm{c}}$ events or the effective $\mathrm{B}^{0} \overline{\mathrm{B}^{0}}$-mixing. These auxiliary parameters are included in the combination procedure in a coherent way. The parameters currently used for combination are:

- the forward-backward-asymmetries $A_{\mathrm{FB}}^{\mathrm{b}}$ and $A_{\mathrm{FB}}^{\mathrm{c}}$ from LEP,

- $\mathcal{A}_{\mathrm{b}}$ and $\mathcal{A}_{\mathrm{c}}$ obtained from the left-right-forward-backward-asymmetries at SLD,

- the normalised partial widths $R_{\mathrm{b}}, R_{\mathrm{c}}$,

- and as auxiliary parameters:

- the fractions of weakly decaying charmed hadrons $f\left(\mathrm{D}^{+}\right), f\left(\mathrm{D}_{\mathrm{s}}\right), f\left(\mathrm{c}_{\mathrm{baryon}}\right)^{8}$,

- the probability that a c-quark fragments into a $\mathrm{D}^{*+}$ that decays into $\mathrm{D}^{0} \pi^{+}$, $\mathrm{P}\left(\mathrm{c} \rightarrow \mathrm{D}^{*+}\right) \times \mathrm{BR}\left(\mathrm{D}^{*+} \rightarrow \pi^{+} \mathrm{D}^{0}\right)$,

- the $\mathrm{B}^{0} \overline{\mathrm{B}^{0}}$ effective mixing parameter $\bar{\chi}$,

- the prompt and cascade semileptonic branching ratio of the b-hadrons $\mathrm{BR}(\mathrm{b} \rightarrow \ell)$ and $\mathrm{BR}(\mathrm{b} \rightarrow \mathrm{c} \rightarrow \ell)$.

The LEP asymmetries are either taken at the three different energies separately to test their energy dependence or are transported to the peak energy before the fit to measure the weak mixing angle, assuming SM energy dependence.

\footnotetext{
${ }^{7}$ If the publications use different parameters than the agreed ones, the results with the common parameter set are made available for combination.

${ }^{8} f\left(\mathrm{D}^{0}\right)$ is calculated from the constraint $f\left(\mathrm{D}^{0}\right)+f\left(\mathrm{D}^{+}\right)+f\left(\mathrm{D}_{\mathrm{s}}\right)+f\left(\mathrm{c}_{\text {baryon }}\right)=1$.
} 
As an input to the combination the experiments provide a table for each analysis giving the result and the error breakdown according to the specified sources. If more than one quantity is measured in the same analysis the correlations are also given. If a result depends on a fit parameter that is not measured in the same analysis, the dependence on this parameter is given as well and the result is modified according to the current value of that parameter in the fit.

For the combination itself a $\chi^{2}$-minimisation is used where the input covariance matrix is constructed from the errors and correlations in the tables provided by the experiments.

\subsection{Combined LEP/SLC results}

Combining all LEP and SLD measurements using the procedure described above yields:

$$
\begin{aligned}
R_{\mathrm{b}}^{0} & =0.21732 \pm 0.00087 \\
R_{\mathrm{c}}^{0} & =0.1731 \pm 0.0044 \\
A_{\mathrm{FB}}^{0, \mathrm{~b}} & =0.0998 \pm 0.0022 \\
A_{\mathrm{FB}}^{0, \mathrm{c}} & =0.0735 \pm 0.0045 \\
\mathcal{A}_{\mathrm{b}} & =0.899 \pm 0.049 \\
\mathcal{A}_{\mathrm{c}} & =0.660 \pm 0.045 \\
\mathrm{BR}(\mathrm{b} \rightarrow \ell) & =0.1106 \pm 0.0019 \\
\mathrm{BR}(\mathrm{b} \rightarrow \mathrm{c} \rightarrow \bar{\ell}) & =0.0802 \pm 0.0032 \\
\bar{\chi} & =0.1215 \pm 0.0042 \\
f\left(\mathrm{D}^{+}\right) & =0.220 \pm 0.020 \\
f\left(\mathrm{D}_{\mathrm{s}}\right) & =0.112 \pm 0.027 \\
f\left(\mathrm{c}_{\mathrm{baryon}}\right) & =0.086 \pm 0.022 \\
\mathrm{P}\left(\mathrm{c} \rightarrow \mathrm{D}^{*+}\right) \times \mathrm{BR}\left(\mathrm{D}^{*+} \rightarrow \pi^{+} \mathrm{D}^{0}\right) & =0.1615 \pm 0.0059
\end{aligned}
$$

with a $\chi^{2} / n d f$ of $60 /(98-13)$. For this fit the LEP asymmetries at the different centre-of-mass energies have all been corrected to pure Z-exchange. The correlations between the electroweak parameters are given in table 7. A detailed error breakdown is shown in table 8 . The $\chi^{2}$ of the fit corresponds to a probability of $98.2 \%$. This indicates very good agreement of the different results. However, as can be seen from table 8, systematic errors play an important role. Since they are normally estimated conservatively a somewhat too small $\chi^{2}$ is not surprising.

Treating the different centre of mass energies separately for the asymmetries one gets

$$
\begin{aligned}
& A_{\mathrm{FB}}^{\mathrm{b}}(89.55 \mathrm{GeV})=0.0556 \pm 0.0088, \\
& A_{\mathrm{FB}}^{\mathrm{c}}(89.55 \mathrm{GeV})=-0.031 \pm 0.018, \\
& A_{\mathrm{FB}}^{\mathrm{b}}(91.26 \mathrm{GeV})=0.0982 \pm 0.0023, \\
& A_{\mathrm{FB}}^{\mathrm{c}}(91.26 \mathrm{GeV})=0.0672 \pm 0.0047, \\
& A_{\mathrm{FB}}^{\mathrm{b}}(92.94 \mathrm{GeV})=0.1085 \pm 0.0077, \\
& A_{\mathrm{FB}}^{\mathrm{c}}(92.94 \mathrm{GeV})=0.136 \pm 0.015,
\end{aligned}
$$

where all correlations are small. The other results remain completely unchanged. 


\begin{tabular}{|l|rrrrrr|}
\hline & $R_{\mathrm{b}}$ & $R_{\mathrm{c}}$ & $A_{\mathrm{FB}}^{0, \mathrm{~b}}$ & $A_{\mathrm{FB}}^{0, \mathrm{c}}$ & $\mathcal{A}_{\mathrm{b}}$ & $\mathcal{A}_{\mathrm{c}}$ \\
\hline$R_{\mathrm{b}}$ & 1.00 & -0.15 & -0.01 & 0.01 & -0.03 & 0.01 \\
$R_{\mathrm{c}}$ & -0.15 & 1.00 & 0.06 & -0.05 & 0.04 & -0.03 \\
$A_{\mathrm{FB}}^{0, \mathrm{~b}}$ & -0.01 & 0.06 & 1.00 & 0.13 & 0.03 & 0.01 \\
$A_{\mathrm{FB}}^{0, \mathrm{c}}$ & 0.01 & -0.05 & 0.13 & 1.00 & 0.00 & 0.05 \\
$\mathcal{A}_{\mathrm{b}}$ & -0.03 & 0.04 & 0.03 & 0.00 & 1.00 & 0.03 \\
$\mathcal{A}_{\mathrm{c}}$ & 0.01 & -0.03 & 0.01 & 0.05 & 0.03 & 1.00 \\
\hline
\end{tabular}

Table 7: The correlation matrix for the LEP and SLD electroweak parameters with heavy flavours.

\begin{tabular}{|l|c|c|c|c|c|c|}
\hline source & $\begin{array}{c}R_{\mathrm{b}}^{0} \\
\cdot 10^{-3}\end{array}$ & $\begin{array}{c}R_{\mathrm{c}}^{0} \\
\cdot 10^{-3}\end{array}$ & $\begin{array}{c}A_{\mathrm{FB}}^{0, \mathrm{~b}} \\
\cdot 10^{-3}\end{array}$ & $\begin{array}{c}A_{\mathrm{FB}}^{0, \mathrm{c}} \\
\cdot 10^{-3}\end{array}$ & $\begin{array}{c}\mathcal{A}_{\mathrm{b}} \\
\cdot 10^{-2}\end{array}$ & $\begin{array}{c}\mathcal{A}_{\mathrm{c}} \\
\cdot 10^{-2}\end{array}$ \\
\hline statistics & 0.53 & 2.8 & 1.9 & 3.9 & 3.9 & 3.8 \\
internal systematics & 0.42 & 2.4 & 0.9 & 2.0 & 3.0 & 2.4 \\
$\mathrm{QCD}$ effects & 0.19 & 0.4 & 0.2 & 0.1 & 0 & 0 \\
$\operatorname{Br}\left(D \rightarrow \mathrm{K}^{0} X\right)$ & 0.15 & 0.2 & 0 & 0 & 0 & 0 \\
$\mathrm{~B}$ decay multiplicity & 0.13 & 0.1 & 0 & 0.2 & 0 & 0 \\
$\mathrm{D}$ decay multiplicity & 0.09 & 0.4 & 0 & 0.1 & 0 & 0 \\
$\operatorname{Br}\left(\mathrm{D}^{0} \rightarrow \mathrm{K}^{-} \pi^{+}\right)$ & 0.05 & 0.1 & 0 & 0 & 0 & 0 \\
$\operatorname{Br}\left(\mathrm{D}^{+} \rightarrow \mathrm{K}^{-} \pi^{+} \pi^{+}\right)$ & 0.10 & 0.4 & 0 & 0 & 0 & 0.1 \\
$\operatorname{Br}\left(\mathrm{D}_{\mathrm{s}} \rightarrow \phi \pi^{+}\right)$ & 0.04 & 1.1 & 0 & 0 & 0 & 0.1 \\
$\mathrm{BR}(\mathrm{c} \rightarrow \ell)$ & 0.05 & 1.4 & 0.2 & 0.2 & 0 & 0.1 \\
gluon splitting & 0.41 & 0.7 & 0 & 0.3 & 0.1 & 0.2 \\
b fragmentation & 0.02 & 0.1 & 0.1 & 0.1 & 0 & 0 \\
c fragmentation & 0.04 & 0.6 & 0.1 & 0.1 & 0.2 & 0.3 \\
light quarks & 0.16 & 0.5 & 0.3 & 0.1 & 0 & 0 \\
Semil. decays & 0.02 & 0.5 & 0.3 & 0.7 & 0.2 & 0.4 \\
\hline total & 0.87 & 4.4 & 2.2 & 4.5 & 4.9 & 4.5 \\
\hline
\end{tabular}

Table 8: Error breakdown of the electroweak observables with heavy quarks. 


\section{$6 \quad$ Interpretation of the Results}

\subsection{Comparison with Standard Model Predictions}

As explained in section 2.3, the peak value of the forward backward asymmetries depends on the weak mixing angle $\sin ^{2} \theta_{\text {eff }}$ whereas the energy dependence is governed by the $\gamma-\mathrm{Z}$ interference term which depends on the axial couplings that are known to a much better accuracy. Figure 19 compares the measured energy dependence of $A_{\mathrm{FB}}^{\mathrm{b}}$ and $A_{\mathrm{FB}}^{\mathrm{c}}$ with the prediction.

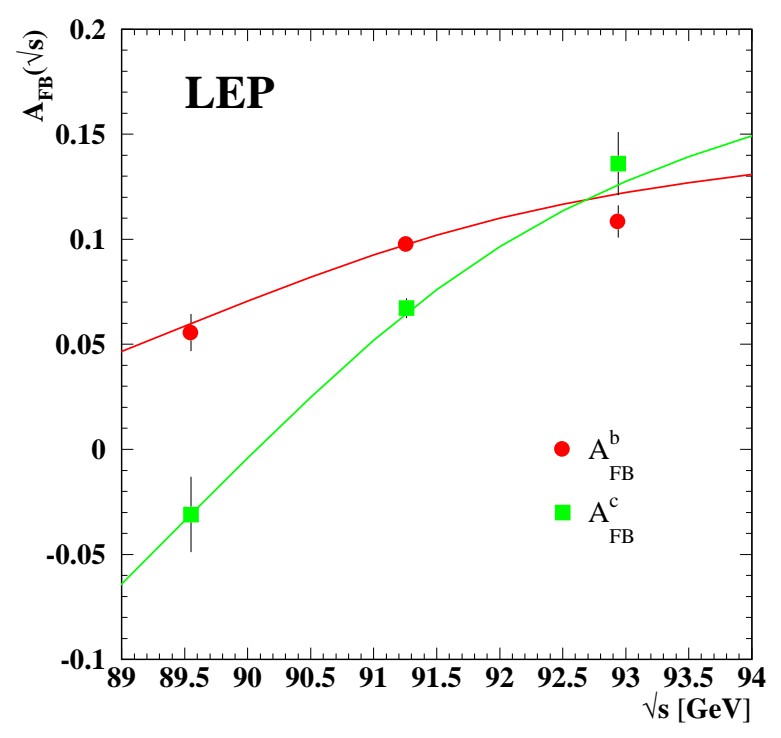

Figure 19: Centre of mass energy dependence of the b- and c-quark forward-backward asymmetry. The solid lines show the Standard Model predictions.

Since the energy dependence is well described by the model it is reasonable to correct all asymmetries to pure Z-exchange.

Figure 20 compares the $95 \%$ probability contours in the $A_{\mathrm{FB}}^{0, \mathrm{~b}}-R_{\mathrm{b}}^{0}$ and $A_{\mathrm{FB}}^{0, \mathrm{c}}-R_{\mathrm{c}}^{0}$ planes with the model prediction. For the prediction, the top quark mass is fixed to its measured value $m_{\mathrm{t}}=173.9 \pm 5.2 \mathrm{GeV}[92,93]^{9}$ and the Higgs mass is varied between $70 \mathrm{GeV}$ and $1 \mathrm{TeV}$. Good agreement with the model prediction can be observed. All numbers agree within one standard deviation apart from $R_{\mathrm{b}}^{0}$ where the agreement is about $1.5 \sigma$.

Unlike the polarised asymmetries, the unpolarised asymmetries depend on the product of the initial and final state coupling parameters. To obtain information on the final state coupling parameters $\mathcal{A}_{\mathrm{b}}$ and $\mathcal{A}_{\mathrm{c}}$, the value of $\mathcal{A}_{\mathrm{e}}$ measured with the leptonic asymmetries at LEP and the left-right asymmetry at SLD has to be used. Figure 21 shows the measured regions in the $\mathcal{A}_{\mathrm{e}}-\mathcal{A}_{\mathrm{b}}$ and $\mathcal{A}_{\mathrm{e}}-\mathcal{A}_{\mathrm{c}}$ planes from the polarised and unpolarised quark asymmetries, the LEP leptonic asymmetries taken from [94], and $A_{\mathrm{LR}}$ from SLD [63] together with the Standard Model prediction. Each measurement individually agrees well with the prediction, but the global agreement is slightly marginal.

\footnotetext{
${ }^{9}$ The $\mathrm{CDF}$ and D0 results have been averaged assuming $2 \mathrm{GeV}$ common systematic error.
} 

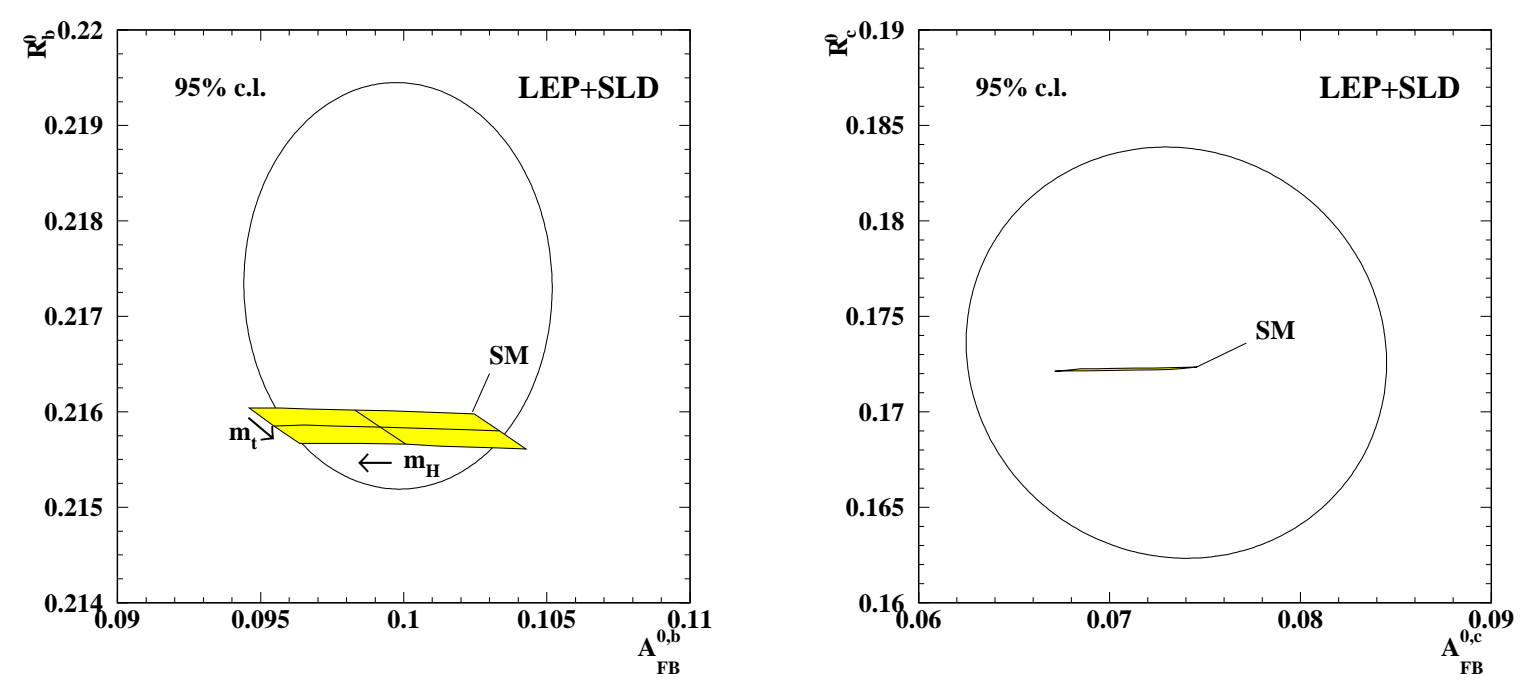

Figure 20: $95 \%$ c.l. contours in the $A_{\mathrm{FB}}^{0, \mathrm{~b}}-R_{\mathrm{b}}^{0}$ (left) and $A_{\mathrm{FB}}^{0, \mathrm{c}}-R_{\mathrm{c}}^{0}$ (right) planes. The areas marked "SM" show the Standard Model prediction for $m_{\mathrm{H}}=300_{-230}^{+700} \mathrm{GeV}$ and $m_{\mathrm{t}}=$ $173.9 \pm 5.2 \mathrm{GeV}$.

\subsection{Quark couplings}

Since the forward backward asymmetries depend on the ratio $g_{V} / g_{A}$ and the partial width on $g_{V}^{2}+g_{A}^{2}$, the couplings can be determined individually from the LEP/SLD heavy flavour results once the $\mathrm{Z}$ leptonic couplings and the hadronic width are known. For pure Z-exchange, all observables are invariant under a sign change and exchange of the vector and axial vector coupling. Both ambiguities can however be resolved using the off-peak asymmetries.

To take fully into account all correlations, the heavy flavour results quoted in section 5.6 are fitted together with all other LEP/SLD observables leaving the leptonic couplings and the band c-quark couplings as free parameters ${ }^{10}$. Lepton universality has been assumed. In addition the b- and c-asymmetries measured at PEP, PETRA and TRISTAN are included in the fit (see table 9).

\begin{tabular}{|c|c|c|c|}
\hline$\sqrt{s}$ & $A_{\mathrm{FB}}^{\mathrm{b}}$ & $A_{\mathrm{FB}}^{\mathrm{c}}$ & Refs. \\
\hline $29 \mathrm{GeV}$ & $-0.052 \pm 0.081$ & $-0.106 \pm 0.026$ & {$[95-100]$} \\
$35 \mathrm{GeV}$ & $-0.214 \pm 0.050$ & $-0.142 \pm 0.024$ & {$[101-106]$} \\
$44 \mathrm{GeV}$ & $-0.460 \pm 0.147$ & $-0.025 \pm 0.087$ & {$[101,102]$} \\
$58 \mathrm{GeV}$ & $-0.588 \pm 0.078$ & $-0.551 \pm 0.077$ & {$[107-111]$} \\
\hline
\end{tabular}

Table 9: $A_{\mathrm{FB}}^{\mathrm{b}}$ and $A_{\mathrm{FB}}^{\mathrm{c}}$ measured at PEP, PETRA and TRISTAN. The b-asymmetries are corrected for mixing using $\bar{\chi}=0.1215$.

The results are summarised in table 10 and, for the quark couplings, displayed in figure 22 . All ambiguities are excluded with more than $99 \%$ confidence level. The low energy data have no significant effect on the central values and errors, however they are needed for the resolution of the ambiguities. The $\chi^{2}$ of the fit is 20 for 17 degrees of freedom, showing good consistency of the data. The fact that the SM prediction of the b-quark couplings falls slightly outside the $95 \%$

\footnotetext{
${ }^{10}$ For technical reasons also the $\mathrm{Z}$ mass, its total and hadronic widths and $\alpha_{s}$ are left free.
} 

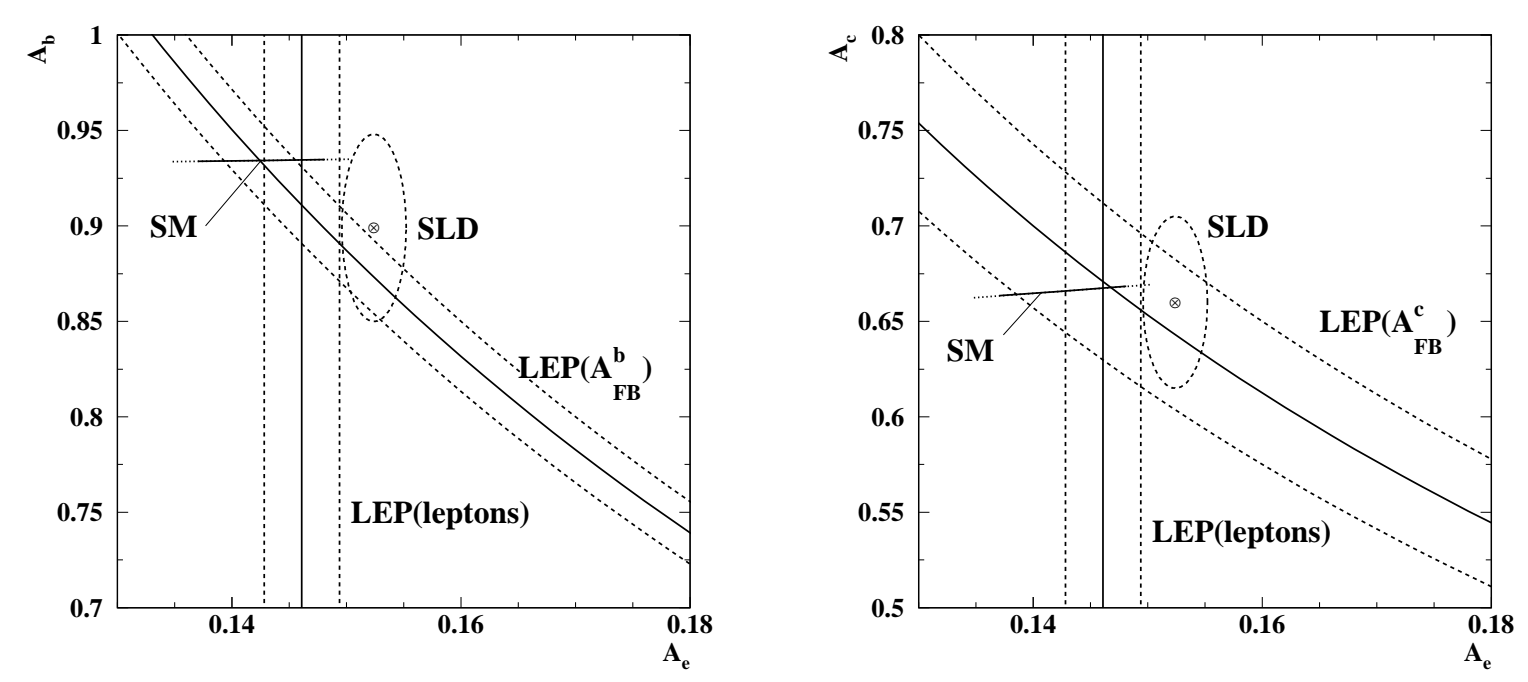

Figure 21: Measurements of $\mathcal{A}_{\mathrm{e}}-\mathcal{A}_{\mathrm{b}}$ (left) and $\mathcal{A}_{\mathrm{e}}-\mathcal{A}_{\mathrm{c}}$ (right) at LEP and SLD. The dashed bands and ellipses correspond to one standard deviation. The solid lines labelled "SM" show the Standard Model prediction for a variation of the Higgs mass between $70 \mathrm{GeV}$ (high $\mathcal{A}_{\mathrm{e}}$ ) and $1 \mathrm{TeV}\left(\right.$ low $\mathcal{A}_{\mathrm{e}}$ ). The dotted line corresponds to the prediction adding the uncertainties on $m_{\mathrm{t}}$ and $\alpha_{Q E D}\left(m_{\mathrm{Z}}^{2}\right)$.

c.l. contour allowed by the data reflects the small disagreement seen in figure 21 and the $1.5 \sigma$ difference between the measurement and the prediction in $R_{\mathrm{b}}^{0}$. The results establish however without any doubt that the b-quark is a down type and the c-quark an up-type member of an isospin doublet.

\begin{tabular}{|c|c|rrrrrr|}
\hline coupling & fit value & \multicolumn{7}{|c|}{ correlations } \\
\hline$g_{V \mathrm{e}}$ & $-0.03766 \pm 0.00052$ & 1.00 & -0.08 & -0.49 & 0.48 & 0.15 & -0.07 \\
$g_{A \mathrm{e}}$ & $-0.50103 \pm 0.00031$ & -0.08 & 1.00 & 0.07 & -0.04 & -0.02 & -0.01 \\
$g_{V \mathrm{~b}}$ & $-0.32002 \pm 0.01196$ & -0.49 & 0.07 & 1.00 & -0.98 & -0.18 & 0.03 \\
$g_{A \mathrm{~b}}$ & $-0.51702 \pm 0.00761$ & 0.48 & -0.04 & -0.98 & 1.00 & 0.18 & -0.01 \\
$g_{V \mathrm{c}}$ & $0.18899 \pm 0.01040$ & 0.15 & -0.02 & -0.18 & 0.18 & 1.00 & -0.35 \\
$g_{A \mathrm{c}}$ & $0.50409 \pm 0.00765$ & -0.07 & -0.01 & 0.03 & -0.01 & -0.35 & 1.00 \\
\hline
\end{tabular}

Table 10: Fit of effective quark and lepton couplings at LEP and SLD.

\subsection{Global consistency with the Standard Model}

As explained in section 2.3, the value of $\mathcal{A}_{\mathrm{b}}$ is predicted by the Standard Model, independently of $m_{\mathrm{t}}$ and $m_{\mathrm{H}}$, and $A_{\mathrm{FB}}^{\mathrm{b}}$ can thus be used as a measurement of $\mathcal{A}_{\mathrm{e}}$ or $\sin ^{2} \theta_{\mathrm{eff}}^{\ell}$. Table 11 summarises all measurements of $\sin ^{2} \theta_{\text {eff }}^{\ell}$ at LEP and SLD [94]. The $\chi^{2}$-probability for the agreement of all results is $16 \%$. The two most precise measurements of $\sin ^{2} \theta_{\mathrm{eff}}^{\ell}, A_{\mathrm{FB}}^{\mathrm{b}}$ and $A_{\mathrm{LR}}$, are 2.5 standard deviations apart. However, since the overall agreement is good and the uncertainties of both measurements are completely dominated by statistics, this difference is interpreted as a statistical fluctuation. 

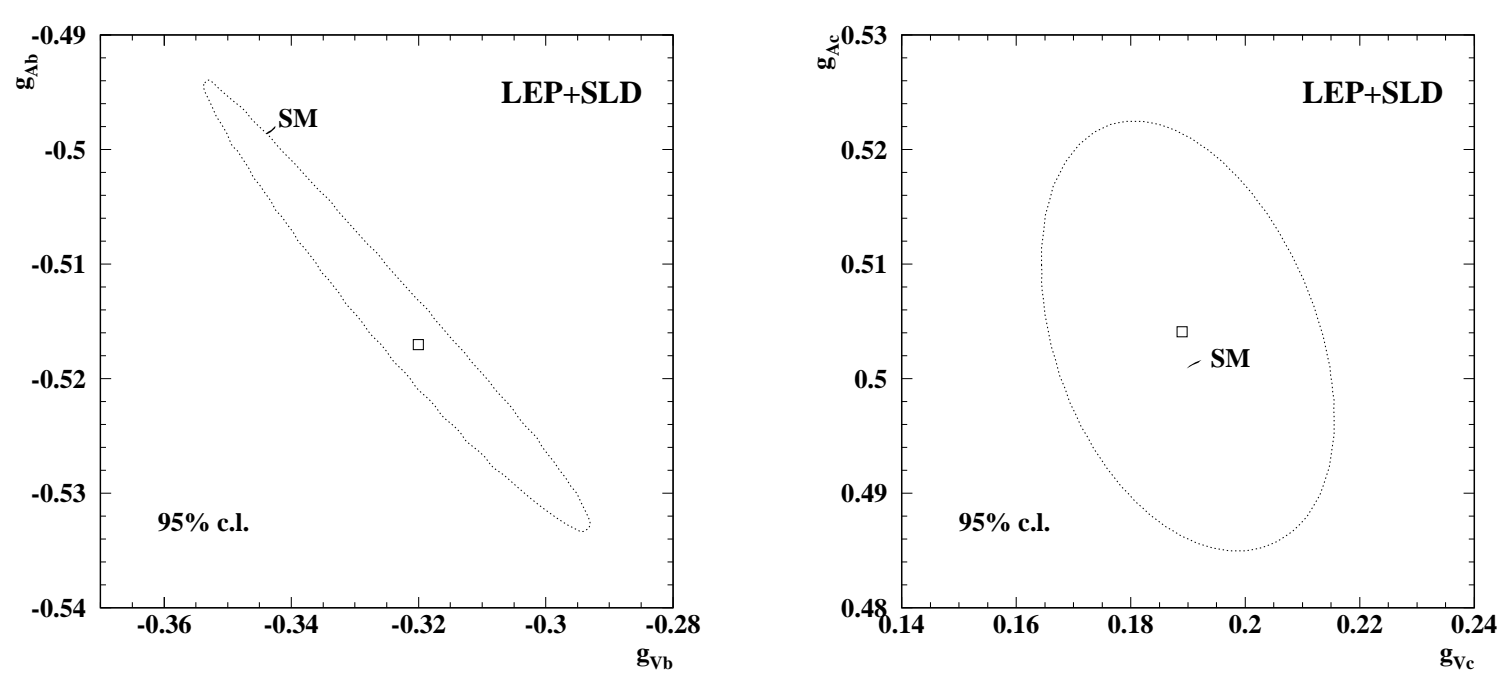

Figure 22: $95 \%$ c.l. contours of the effective couplings for b and c quarks compared with the Standard Model predictions.

\begin{tabular}{|l|l|c|}
\hline & \multicolumn{1}{|c|}{$\sin ^{2} \theta_{\text {eff }}^{\ell}$} & Pull \\
\hline \hline$A_{\mathrm{FB}}^{0, \mathrm{~b}}$ & $0.23211 \pm 0.00039$ & +1.6 \\
$A_{\mathrm{FB}}^{0, \mathrm{c}}$ & $0.23155 \pm 0.00104$ & 0 \\
\hline$A_{\mathrm{FB}}^{0, \ell}$ & $0.23102 \pm 0.00056$ & -0.9 \\
$\mathcal{A}_{\tau}$ & $0.23228 \pm 0.00081$ & +1.0 \\
$\mathcal{A}_{\mathrm{e}}$ & $0.23243 \pm 0.00093$ & +1.0 \\
\hline$\left\langle Q_{\mathrm{FB}}\right\rangle$ & $0.2322 \pm 0.0010$ & +0.7 \\
\hline$A_{\mathrm{LR}}$ (SLD) & $0.23084 \pm 0.00035$ & -1.9 \\
\hline average & $0.23150 \pm 0.00021$ & \\
\hline
\end{tabular}

Table 11: Measurements of $\sin ^{2} \theta_{\text {eff }}^{\ell}$ at LEP and SLD.

All lineshape results and leptonic asymmetries measured at LEP and SLD can be summarised in terms of the $\mathrm{Z}$ mass, its partial decay width into leptons, hadrons and invisible particles, and the leptonic coupling parameter $\mathcal{A}_{\mathrm{e}}$. The values used in the following are taken from $[63,94]$ and are shown in table 12.

To test the overall agreement of the data with each other and with the Standard Model, a fit is performed to all data leaving $m_{\mathrm{t}}, m_{\mathrm{H}}$ and the strong coupling constant $\alpha_{s}\left(m_{\mathrm{Z}}^{2}\right)$ as free parameters. In addition to the heavy flavour data and the data from table $12, m_{\mathrm{t}}=$ $173.9 \pm 5.2 \mathrm{GeV}$ and $\alpha_{Q E D}\left(m_{\mathrm{Z}}^{2}\right)=1 /(128.896 \pm 0.090)[15]$ were used. The result of the fit is

$$
\begin{aligned}
m_{\mathrm{t}} & =170.66 \pm 5.0 \mathrm{GeV} \\
m_{\mathrm{H}} & =68_{-43}^{+87} \mathrm{GeV} \\
\alpha_{s}\left(m_{\mathrm{Z}}^{2}\right) & =0.120 \pm 0.003
\end{aligned}
$$

with a $\chi^{2} / n d f=8.5 / 8$, indicating good overall agreement. If $\alpha_{Q E D}\left(m_{\mathrm{Z}}^{2}\right)=1 /(128.928 \pm 0.023)$ from [17] is used instead, the preferred value of the Higgs mass becomes $m_{\mathrm{H}}=83_{-39}^{+64} \mathrm{GeV}$. For the interpretation of the $m_{\mathrm{H}}$ error, it should be noted that the error on $\log \left(m_{\mathrm{H}}\right)$ is approximately Gaussian. The fitted value of $\alpha_{s}\left(m_{\mathrm{Z}}^{2}\right)$ is well compatible with the world average 


\begin{tabular}{|c|c|rrrrr|}
\hline Parameter & value & \multicolumn{5}{|c|}{ correlations } \\
\hline$m_{\mathrm{Z}}$ & $91.1867 \pm 0.0020 \mathrm{GeV}$ & 1.00 & 0.03 & 0.06 & 0.01 & 0.03 \\
$\Gamma_{\text {had }}$ & $1743.1 \pm 2.3 \mathrm{MeV}$ & 0.03 & 1.00 & 0.46 & -0.42 & 0.00 \\
$\Gamma_{\ell}$ & $83.91 \pm 0.10 \mathrm{MeV}$ & 0.06 & 0.46 & 1.00 & 0.24 & 0.00 \\
$\Gamma_{\text {inv }}$ & $500.2 \pm 1.9 \mathrm{MeV}$ & 0.01 & -0.42 & 0.24 & 1.00 & 0.00 \\
$\mathcal{A}_{\mathrm{e}}$ & $0.1498 \pm 0.0021$ & 0.03 & 0.00 & 0.00 & 0.00 & 1.00 \\
\hline
\end{tabular}

Table 12: Observables from the Z-lineshape and leptonic asymmetry measurements at LEP and SLD.

$\alpha_{s}\left(m_{\mathrm{Z}}^{2}\right)=0.118 \pm 0.003$ [6], and the Higgs mass $m_{\mathrm{H}}$ with the limit from direct searches, $m_{\mathrm{H}}>89.3 \mathrm{GeV}$ [112]. If the Standard Model is required to be valid up to the Planck scale the Higgs mass has to be in the range $m_{\mathrm{H}} \sim 170 \pm 20 \mathrm{GeV}$ [113], which is also perfectly compatible with the fit results.

\subsection{Model independent parametrisation}

As explained in section 2.2 all loop corrections can be parametrised in a model independent way using the parameters $\Delta \rho_{\mathrm{f}}$ and $\sin ^{2} \theta_{\text {eff }}^{\mathrm{f}}$. Several other parametrisations exist where the quadratic terms originating from fermion loops are absorbed into one parameter. The most commonly used parameter sets are the S, T, and U parameters [114] and the $\varepsilon$ parameters [115], respectively, which are basically equivalent. The $\varepsilon$ parameters are defined as $^{11}$

$$
\begin{aligned}
\Gamma_{\ell} & =\left(\Gamma_{\ell}\right)_{\text {Born }}\left(1+1.20 \varepsilon_{1}-0.26 \varepsilon_{3}\right) \\
A_{\mathrm{FB}}^{0, \ell} & =\left(A_{\mathrm{FB}}^{0, \ell}\right)_{\text {Born }}\left(1+34.72 \varepsilon_{1}-45.15 \varepsilon_{3}\right) \\
\Gamma_{\mathrm{b}} & =\left(\Gamma_{\mathrm{b}}\right)_{\text {Born }}\left(1+1.42 \varepsilon_{1}-0.54 \varepsilon_{3}+2.29 \varepsilon_{\mathrm{b}}\right),
\end{aligned}
$$

where QCD corrections and the running of $\alpha_{Q E D}$ are already included in the Born terms. The $\varepsilon$ parameters can also be expressed in terms of $\Delta \rho$ and $\sin ^{2} \theta_{\text {eff }}$ :

$$
\begin{aligned}
\Delta \rho_{\ell} & =\varepsilon_{1} \\
\sin ^{2} \theta_{\mathrm{eff}}^{\ell} & =\frac{1}{2}\left(1-\sqrt{1-\frac{4 \pi \alpha_{Q E D}\left(m_{\mathrm{Z}}^{2}\right)}{\sqrt{2} G_{\mathrm{F}} m_{\mathrm{Z}}^{2}}}\right)\left(1-1.43 \varepsilon_{1}+1.86 \varepsilon_{3}\right) \\
\Delta \rho_{\mathrm{b}}-\Delta \rho_{\mathrm{d}} & =2 \varepsilon_{\mathrm{b}}
\end{aligned}
$$

The absence of extra parameters describing the light quark vertices comes from the prejudice, that these vertices do not contain any new physics.

For the determinations of these parameters different assumptions have been made:

a) $\varepsilon_{1}$ and $\varepsilon_{3}$ are determined only by the leptonic variables, $\varepsilon_{\mathrm{b}}$ is obtained from $\Gamma_{\mathrm{b}}=\Gamma_{\mathrm{had}} R_{\mathrm{b}}^{0}$. This makes no assumptions on the light quark vertices.

b) In addition, the hadronic vertices for the non b quarks have been taken from the Standard Model and the quark asymmetries have been added.

c) The b-vertex has also been taken from the Standard Model, assuming $m_{\mathrm{t}}=173.9 \pm$ $5.2 \mathrm{GeV}$.

\footnotetext{
${ }^{11} \varepsilon_{2}$ is defined using the W-mass which is not discussed here.
} 
In all cases, $\alpha_{s}$ has been constrained to $\alpha_{s}\left(m_{\mathrm{Z}}^{2}\right)=0.118 \pm 0.003$ and $\alpha_{Q E D}\left(m_{\mathrm{Z}}^{2}\right)$ to $\alpha_{Q E D}\left(m_{\mathrm{Z}}^{2}\right)=$ $1 /(128.896 \pm 0.090)$. $\varepsilon_{\mathrm{b}}$ can also be obtained from $R_{\mathrm{b}}^{0}$ alone, assuming no new physics in the light quark vertices. The results of all fits are shown in table 13. Figure 23 compares the results of the fits with the Standard Model predictions in the $\varepsilon_{1}-\varepsilon_{3}$ and $\varepsilon_{1}-\varepsilon_{\mathrm{b}}$ planes. For a not too heavy Higgs, the data agree well with the model prediction.

\begin{tabular}{|c|c|c|c|}
\hline Fit & $\varepsilon_{1} \cdot 10^{3}$ & $\varepsilon_{3} \cdot 10^{3}$ & $\varepsilon_{\mathrm{b}} \cdot 10^{3}$ \\
\hline Leptonic variables plus $\Gamma_{\mathrm{b}}$ & $4.1 \pm 1.3$ & $3.5 \pm 1.3$ & $-2.7 \pm 1.9$ \\
Leptonic plus hadronic variables & $4.0 \pm 1.2$ & $4.0 \pm 1.2$ & $-3.1 \pm 1.8$ \\
$\mathrm{~b} \overline{\mathrm{b}}$ vertex also fixed & $4.5 \pm 1.1$ & $4.4 \pm 1.2$ & \\
$R_{\mathrm{b}}^{0}$ only & & & $-2.2 \pm 2.2$ \\
\hline
\end{tabular}

Table 13: Fit of the $\varepsilon$ parameters. The details of the fit are described in the text. The correlation between $\varepsilon_{1}$ and $\varepsilon_{3}$ is around $80 \%$ whereas the other correlations are small. For the determination of $\varepsilon_{\mathrm{b}}$ from $R_{\mathrm{b}}^{0}$ alone, the small dependence on $\varepsilon_{1,3}$ has been taken from the first fit.
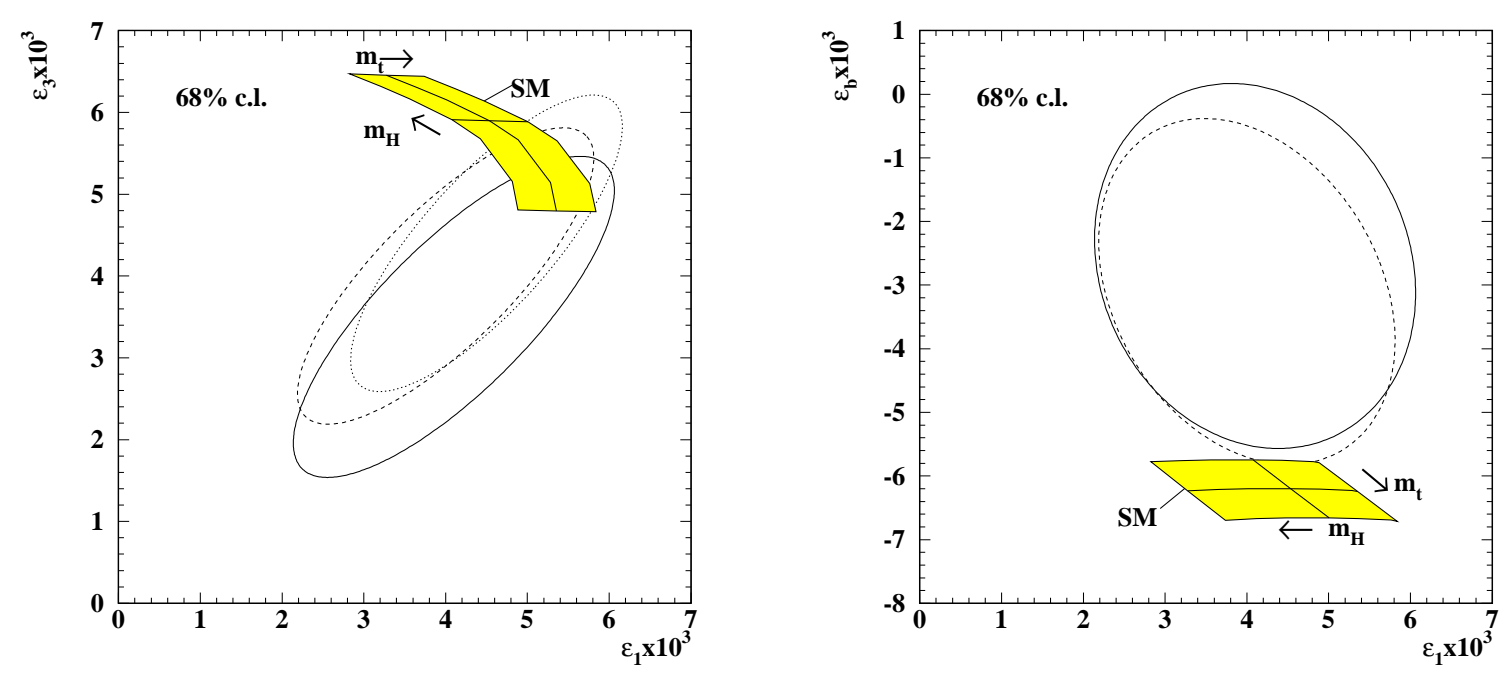

Figure 23: $68 \%$ c.l. contours in the $\varepsilon_{1}-\varepsilon_{3}$ (left) and $\varepsilon_{1}-\varepsilon_{\mathrm{b}}$ (right) planes. The solid ellipses represent a fit to the leptonic observables and $\Gamma_{\mathrm{b}}$. For the fit shown by the dashed ellipses the hadronic and heavy flavour observables have also been used. In the fit represented by the dotted ellipse the bb-vertex is also fixed to its Standard Model prediction. The areas marked "SM" show the Standard Model prediction for $m_{\mathrm{H}}=300_{-230}^{+700} \mathrm{GeV}$ and $m_{\mathrm{t}}=173.9 \pm 5.2 \mathrm{GeV}$.

\subsection{Constraints on new Physics}

Supersymmetry [116] is a decoupling theory which predicts, in its minimal version, that the lightest Higgs Boson has a mass below about $130 \mathrm{GeV}[117]^{12}$. The decoupling means that, if the supersymmetric particles are sufficiently heavy, the $\mathrm{Z}$ precision data cannot distinguish between supersymmetry and the Standard Model with a similarly light Higgs. In the past,

\footnotetext{
${ }^{12}$ Non-minimal supersymmetric models also tend to predict light Higgses.
} 
indications that $R_{\mathrm{b}}$ was larger than the SM prediction have been reported. In this context, it was shown that vertex loops involving charginos or stops could give measurable contributions to $R_{\mathrm{b}}$. However all these solutions require new particles that should have been discovered at LEP-II by now [18].

The situation is different for technicolour models [116]. Technifermions should contribute to the $\varepsilon$ parameters like normal fermions, even if they are heavy and degenerate within a doublet. Figure 24 shows the $95 \%$ confidence level contours in the $\varepsilon_{1}-\varepsilon_{3}$ and $\varepsilon_{1}-\varepsilon_{\mathrm{b}}$ planes compared with the prediction of a one generation technicolour model with Majorana technineutrinos and degenerate techniquarks as discussed in [118]. The model is clearly inconsistent with the data and the agreement is made worse by allowing for more generations, a mass splitting for the techniquarks, or a Dirac technineutrino. However technicolour models with additional assumptions and some fine tuning exist that cannot be excluded by measurements on the $\mathrm{Z}[119]$.
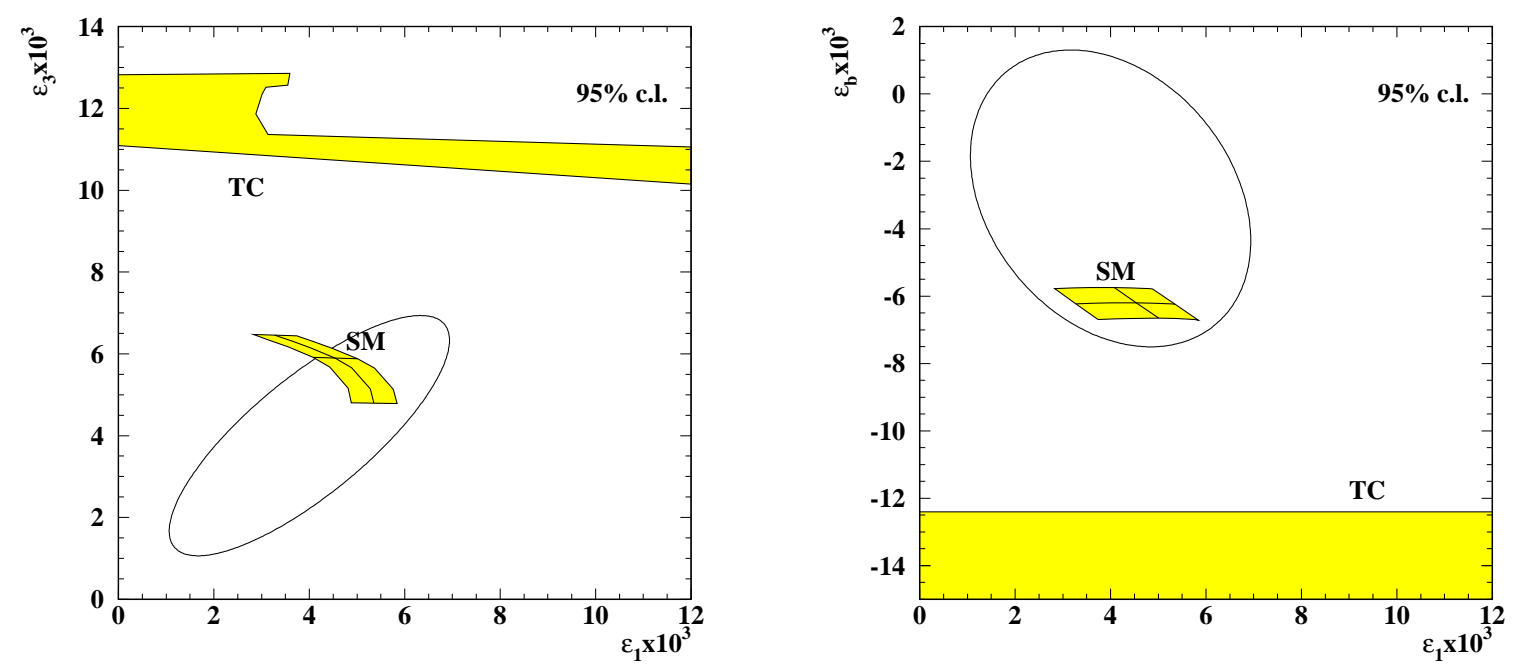

Figure 24: $95 \%$ c.l. contours in the $\varepsilon_{1}-\varepsilon_{3}$ (left) and $\varepsilon_{1}-\varepsilon_{\mathrm{b}}$ (right) planes. The solid ellipses represent a fit to the leptonic and hadronic observables corresponding to the dashed ellipses in figure 23. The areas labeled "SM" correspond to the Standard Model prediction and the areas labeled "TC" to the technicolour model with Majorana technineutrinos described in [118].

For models that predict new physics on the Born level, the formalism of effective couplings cannot be used but individual observables have to be used instead. To explain the disagreement between $A_{\mathrm{LR}}$ and $A_{\mathrm{FB}}^{\mathrm{b}}$ and the former deviation of $R_{\mathrm{b}}$, models with a $\mathrm{Z}^{\prime}$ [120] or an R-parity violating sneutrino [77] have been proposed where the new particle is almost degenerate with the $\mathrm{Z}$. Due to the different relative couplings of the $\mathrm{Z}$ and the new particle to the different fermions, the measured $\sin ^{2} \theta_{\mathrm{eff}}^{\ell}$-values no longer need to coincide. However the agreement of the energy dependence of the asymmetries and of $R_{\mathrm{b}}$ with the Standard Model prediction constrains the mass and width of the new particle to be almost identical to those of the Z [77].

Extended technicolour models also predict a heavy $Z^{\prime}$. Models of this type have been built that were able to explain the deviation in $R_{\mathrm{b}}$ [121]. Since, however, these models are not able to explain different values of $\sin ^{2} \theta_{\text {eff }}^{\ell}$ obtained from different processes, they do not, at present, describe the data better than the Standard Model. 


\subsection{Prospects for the Future}

The LEP-I analyses presented here are very close to their final state and only a few refinements can be expected. SLD is about to finish a high statistics run which should improve their errors by about a factor 1.5 to 2 compared to the results presented here. The decreased error on $A_{\mathrm{LR}}$ should show whether the disagreement between $\sin ^{2} \theta_{\mathrm{eff}}^{\ell}$ from $A_{\mathrm{LR}}$ and $A_{\mathrm{FB}}^{\mathrm{b}}$ can be explained as a statistical fluctuation. If the discrepancy cannot be explained by this, $\mathcal{A}_{\mathrm{b}}$ from the left-rightforward-backward asymmetry should help to decide if the difference is due to new physics or a mistake in one of the measurements.

LEP is currently running at energies above the $\mathrm{W}$-pair threshold. A precise measurement of the W-mass gives access to the $\varepsilon_{2}$ parameter, adding another dimension to the Standard Model tests. In addition many models which were tested with the precision observables can also be probed directly by discovering or excluding new particles. The heavy quark production rates and asymmetries will be measured with a much worse precision than at LEP-I. However they still help to improve bounds on heavy Z's [122].

\section{Conclusions}

Using about 16 million $\mathrm{Z}$ decays at LEP and up to $300000 \mathrm{Z}$ decays with polarised beams at SLC, the b- and c-quark partial widths and forward-backward asymmetries have been measured at the percent level or better. The quark-asymmetries at LEP are sensitive to the product of the initial state electron and the final state quark couplings, and are among the most sensitive measurements of the effective weak mixing angle $\sin ^{2} \theta_{\mathrm{eff}}^{\ell}$. On the contrary, the left-rightforward-backward asymmetries measured with polarised beams at SLC and the partial widths are sensitive to quark couplings only.

All results agree well with the predictions of the Standard Model, significantly constraining models of possible new physics. Especially models like technicolour, that do not decouple for heavy new particles, need a large amount of fine tuning to stay consistent with the data. The electroweak precision data favour a relatively light Higgs Boson, being equally consistent with supersymmetric extensions and with a Standard Model valid up to the Planck scale.

\section{Acknowledgements}

A review like this is clearly not possible without the many people, running the accelerators and experiments and doing the analyses described here, whom I all wish to thank. Also I am grateful to my colleagues in the LEP heavy flavour working group for their effort combining the data and defining the common input parameters. I thank P. Antilogus, T. Behnke, T. Riemann, R. Tenchini, Su Dong and W. Venus for useful discussions and for reading the manuscript.

Especially, however, I wish to thank J. Drees and D. Treille for all their support and encouragement during my work in DELPHI. 


\section{References}

[1] S. Glashow, Nucl. Phys. 22 (1961) 579;

S. Weinberg, Phys. Rev. Lett. 19 (1967) 1264;

A. Salam, Proc. 8th Nobel Symposium Asglaegaden (1968) 367.

[2] J. Timmermans, Precision tests of the electroweak theory in $e^{+} e^{-}$-interactions, 18th International Symposium on Lepton-Photon Interactions: LP '97, Hamburg, Germany, 28 Jul -1 Aug 1997.

[3] C. Dionisi, Searches for New Particles, 18th International Symposium on Lepton-Photon Interactions: LP '97, Hamburg, Germany, 28 Jul -1 Aug 1997, CERN-PPE-97-158.

[4] G. Quast, Measurement of the Z Boson Parameters at LEP, to be published in Prog. in Part. and Nucl. Phys.

[5] M. Consoli and W. Hollik, Electroweak radiative corrections for Z physics, CERN 89-08, Vol. 1,7 .

[6] Particle Data Group, M. Barnett et al., Phys. Rev. D54 (1996) 1.

[7] A. Sirlin, Phys. Rev. D22 (1980) 2695.

[8] S. G. Goryshny, A. L. Kataev, S. A. Larin, Phys. Lett. B259 (1991) 144;

L. R. Surguladze, M. A. Samuel, Phys. Rev. Lett. 66 (1991) 560.

[9] T. Sjostrand, Comput. Phys. Commun. 82 (1994) 74.

[10] G. Marchesini et al., Comput. Phys. Commun. 67 (1992) 465.

[11] B. Lampe, A Note on QCD corrections to the forward-backward asymmetry of the $b$ quark using thrust to determine the $b$ quark direction, MPI-PH-93-74.

[12] M. Consoli and A. Sirlin, The role of one-loop electroweak effects in $e^{+} e^{-} \rightarrow \mu^{+} \mu^{-}$, CERN 86-02, 63.

[13] A. A. Akhundov, D. Y. Bardin, and T. Riemann, Nucl. Phys. B276 (1986) 1.

[14] W. Beenakker and W. Hollik, Z. Phys. C40 (1988) 141.

[15] S. Eidelmann and F. Jegerlehner, Zeit. Phys. C67 (1995) 585.

[16] M. Davier and A. Höcker, Phys. Lett. B419 (1998) 419.

[17] J. H. Kühn and M. Steinhauser, A Theory driven analysis of the effective QED coupling at $M(Z)$, hep-ph/9802241.

[18] J. Ellis, J. L. Lopez, and D. V. Nanopoulos, Phys. Lett. B397 (1997) 88.

[19] T. Riemann, Phys. Lett. B293 (1992) 451.

[20] M. Cvetic and B. W. Lynn, Phys. Rev. D35 (1987) 51. 
[21] D. Bardin et al., Zeit. Phys. C44 (1989) 493; Comp. Phys. Comm. 59 (1990) 303; Nucl. Phys. B351(1991) 1; Phys. Lett. B255 (1991) 290 and CERN-TH 6443/92 (May 1992).

[22] D. Bardin et al., Electroweak Working Group Report, CERN 95-03, 7 and references therein.

[23] D. Bardin and G. Passarino, Upgrading of precision calculations for electroweak observables, hep-ph/9803425.

[24] S. Myers, The LEP collider, from design to approval and commissioning, CERN 91-08.

[25] P. Emma, The Stanford Linear Collider, Proceedings of the 16th IEEE Particle Accelerator Conference (PAC 95) and International Conference on High Energy Accelerators, Dallas, Texas, 1-5 May 1995, 606.

[26] ALEPH Collaboration, D. Decamp et al., Nucl. Instrum. Meth. A294 (1990) 121.

[27] ALEPH Collaboration, D. Buskulic et al., Nucl. Instrum. Meth. A360 (1995) 481.

[28] DELPHI Collaboration, P. Aarnio et al., Nucl. Instrum. Meth. A303 (1991) 233.

[29] DELPHI Collaboration, P. Abreu et al., Nucl. Instrum. Meth. A378 (1996) 57.

[30] L3 Collaboration, O. Adriani et al., Phys. Rep. 236 (1993) 1.

[31] OPAL Collaboration, K. Ahmet et al., Nucl. Instrum. Meth. A305 (1991) 275.

[32] MARK II Collaboration, G. S. Abrams et al., Nucl. Instrum. Meth. A281 (1989) 55.

[33] SLD Collaboration, G. Agnew et al., SLD Design Report, SLAC-0273.

[34] G. Batigani et al., 1991 IEEE Nuclear Science Symposium, Santa Fee, IEEE transactions on nuclear science, v.NS 39(4-5) (1992) Vol. 1, 438.

[35] DELPHI Collaboration, V. Chabaud et al., Nucl. Instrum. Meth. A368 (1996) 314.

[36] L3 SMD Collaboration, M. Acciarri et al., Nucl. Instrum. Meth. A360 (1995) 103.

[37] P. P. Allport et al., Nucl. Instrum. Meth. A346 (1994) 476.

[38] SLD/VXD3 Group, K. Abe et al., Nucl. Instrum. Meth. A386 (1997) 46.

[39] CERN Application Software Group, GEANT, Detector Description and Simulation Tool, CERN Program Library Long Writeup W5013, http://wwwinfo.cern.ch/asdoc/geant_html3/geantall.html.

[40] OPAL Collaboration, G. Alexander et al., Zeit. Phys. C70 (1996) 357.

[41] ALEPH Collaboration, R. Barate et al., Phys. Lett. B401 (1997) 150.

[42] DELPHI Collaboration, Measurement of the partial decay width $R_{b}^{0}=\Gamma_{b \bar{b}} / \Gamma_{\text {had }}$ with the DELPHI detector at LEP, DELPHI 97-106 CONF 88, contributed paper to the EPS-HEP-97, Jerusalem, EPS-419. 
[43] L3 Collaboration, Measurement of the Z Branching Fraction into Bottom Quarks Using Double Tag Methods, L3 Note 2114, contributed paper to the EPS-HEP-97, Jerusalem, EPS-489.

[44] OPAL Collaboration, K. Ackerstaff et al., Zeit. Phys. C74 (1997) 1.

[45] SLD Collaboration, A Preliminary Measurement of $R_{b}$ using the Upgrade SLD Vertex Detector, SLAC-PUB-7585, contributed paper to the EPS-HEP-97, Jerusalem, EPS-118.

[46] DELPHI Collaboration, Study of Exclusive $D^{*+}$ Production in $Z$ Decays DELPHI 96-109 CONF 36 contributed paper to ICHEP96, Warsaw, 25-31 July 1996 PA01-058.

[47] ALEPH Collaboration, Study of Charmed Hadron Production in Z Decays, contributed paper to the EPS-HEP-97, Jerusalem, EPS-623.

[48] DELPHI Collaboration, Determination of $P\left(c \rightarrow D^{*+}\right)$ and $B R(c \rightarrow \ell)$ at LEP DELPHI 96-106 CONF 33 contributed paper to ICHEP96, Warsaw, 25-31 July 1996 PA01-063.

[49] OPAL Collaboration, K. Ackerstaff et al., Eur. Phys. J. C1 (1998) 439.

[50] ALEPH Collaboration, D. Buskulic et al., Phys. Lett. B384 (1996) 414.

[51] L3 Collaboration, Measurement of the $e^{-} e^{-} \rightarrow Z \rightarrow b \bar{b}$ Forward-Backward Asymmetry Using Leptons, L3 Note 2112, contributed paper to the EPS-HEP-97, Jerusalem, EPS-490.

[52] DELPHI Collaboration, P.Abreu et al., Zeit. Phys C65 (1995) 569;

DELPHI Collaboration, P.Abreu et al., Zeit. Phys C66 (1995) 341;

DELPHI Collaboration, Measurement of the Forward-Backward Asymmetries of $e^{+} e^{-} \rightarrow Z \rightarrow b \bar{b}$ and $e^{+} e^{-} \rightarrow Z \rightarrow c \bar{c}$, DELPHI 95-87 PHYS 522, contributed paper to EPS-HEP-95 Brussels eps0571.

Delphi notes are available at http://wwwcn.cern.ch/〜 pubxx/www/delsec/delnote/.

[53] OPAL Collaboration, R. Akers et al., Updated Measurement of the Heavy Quark Forward-Backward Asymmetries and Average B Mixing Using Leptons in Multihadronic Events, OPAL Physics Note PN226 contributed paper to ICHEP96, Warsaw, 25-31 July 1996 PA05-007.

[54] SLD Collaboration, Measurement of $\mathcal{A}_{b}$ and $\mathcal{A}_{c}$ at the $Z$ using a lepton tag, SLAC-PUB-7637, contributed paper to the EPS-HEP-97, Jerusalem, EPS-124.

[55] OPAL Collaboration, G. Alexander et al., Zeit. Phys. C73 (1997) 379.

[56] ALEPH Collaboration, The Forward-Backward Asymmetry for Charm Quarks at the Z Pole ALEPH 98-020 CONF 98-010.

[57] MAC Collaboration, W. W. Ash et al., Phys. Rev. Lett. 58 (1987) 1080.

[58] ALEPH Collaboration, R. Barate et al., Determination of $A_{F B}^{b}$ using jet charge measurements in $Z$ decays, CERN-EP/98-38 submitted to Phys. Lett. B. 
[59] L3 Collaboration, Afb(bb) using a jet-charge technique on 1994 data, L3 Note 2129.

[60] OPAL Collaboration, K. Ackerstaff et al., Zeit. Phys. C75 (1997) 385.

[61] SLD Collaboration, Measurement of $\mathcal{A}_{b}$ at the $Z$ resonance using a Jet-Charge Technique, SLAC-PUB-7629, contributed paper to the EPS-HEP-97, Jerusalem, EPS-122.

[62] SLD Collaboration, Measurement of $\mathcal{A}_{b}$ from left-right forward-backward asymmetry in $Z$ decays using charged Kaon tagging, SLAC-PUB-7630, contributed paper to the EPS-HEP-97, Jerusalem, EPS-123.

[63] G. Crawford, Precision Electroweak Tests Recent Result from SLD, XXXIIInd Rencontres de Moriond, Les Arcs, France, 14-21 March 1998.

[64] SLD Collaboration, A Preliminary Inclusive Measurement of $\mathcal{A}_{c}$ using the SLD Detector, SLAC-PUB-7595, contributed paper to the EPS-HEP-97, Jerusalem, EPS-126.

[65] D. Abbaneo et al., QCD corrections to the forward-backward asymmetries of $c$ and $b$ quarks at the $Z$ pole, CERN-EP/98-32 submitted to Eur. Phys. J. C.

[66] OPAL Collaboration, R. Akers et al., Phys. Lett. B353 (1995) 595.

[67] M. H. Seymour, Nucl. Phys. B436 (1995) 163.

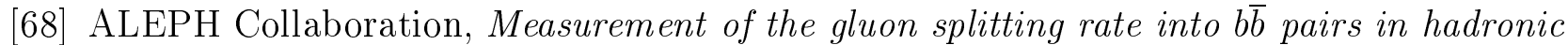
$Z$ decays, contributed paper to the EPS-HEP-97, Jerusalem, EPS-606.

[69] DELPHI Collaboration, P. Abreu et al., Phys. Lett. B405 (1997) 202.

[70] MARK III Collaboration, D. Coffman et al., Phys. Lett. B263 (1991) 135.

[71] The LEP Electroweak Working Group, Presentation of LEP Electroweak Heavy Flavour Results for Summer 1996 Conferences, LEPHF/96-01, available from http://www.cern.ch/LEPEWWG/heavy/.

[72] P. Nason and B. R. Webber, Phys. Lett. B395 (1997) 355.

[73] P. Nason and C. Oleari, Phys. Lett. B387 (1996) 623.

[74] P. Nason and C. Oleari, Phys. Lett. B407 (1997) 57.

[75] ALEPH Collaboration, R. Barate et al., Phys. Lett. B401 (1997) 163.

[76] O. Adriani et al., Phys. Lett. B307 (1993) 237.

[77] J. Erler, J. L. Feng, and N. Polonsky, Phys. Rev. Lett. 78 (1997) 3063.

[78] SLD Collaboration, A Preliminary Measurement of $R_{c}$ with the SLD Detector, SLAC-PUB-7594, contributed paper to the EPS-HEP-97, Jerusalem, EPS-120.

[79] ALEPH Collaboration, R. Barate et al., Measurement of the fraction of hadronic Z decays into charm quark pairs, CERN-EP/98-35 submitted to Eur. Phys. J. C. 
[80] DELPHI Collaboration, Summary of $R_{c}$ measurements in DELPHI, DELPHI 96-110 CONF 37 contributed paper to ICHEP96, Warsaw, 25-31 July 1996 PA01-060.

[81] OPAL Collaboration, G. Alexander et al., Zeit. Phys. C72 (1996) 1.

[82] The LEP collaborations, Nucl. Instrum. Meth. A378 (1996) 101.

[83] DELPHI Collaboration, Determination of $B R(c \rightarrow \ell)$ at LEP DELPHI 97-124 CONF 105 contributed paper to the EPS-HEP-97, Jerusalem, EPS-421.

[84] Particle Data Group, M. Barnett et al., 1997 off-year partial update for the 1998 edition available on the PDG WWW pages (URL: http://pdg.lbl.gov/).

[85] ALEPH Collaboration, D. Buskulic et al., Zeit. Phys. C62 (1994) 179.

[86] DELPHI Collaboration, P. Abreu et al., Zeit. Phys. C66 (1995) 323.

[87] L3 Collaboration, L3 Results on $R_{\mathrm{b}}$ and $\mathrm{BR}(\mathrm{b} \rightarrow \ell)$ for the Glasgow Conference, L3 Note 1625;

L3 Collaboration, M. Acciarri et al., Z Phys. C71 379 (1996);

L3 Collaboration, Measurement of the $B^{0}-\bar{B}^{0}$ Mixing Parameter, L3 Note 2113, contributed paper to the EPS-HEP-97, Jerusalem, EPS-490.

[88] OPAL Collaboration, R. Akers et al., Zeit. Phys. C60 (1993) 199.

[89] ALEPH Collaboration., D. Buskulic et al., Measurement of the semileptonic b branching ratios from inclusive leptons in $Z$ decays, Contributed Paper to EPS-HEP-95 Brussels, eps0404.

[90] DELPHI Collaboration, Measurement of the semileptonic $b$ branching ratios and $\bar{\chi}_{b}$ from inclusive leptons in $Z$ decays, DELPHI 97-118 CONF 100 contributed paper to the EPS-HEP-97, Jerusalem, EPS-415.

[91] OPAL Collaboration, Measurement of the semileptonic branching fraction of inclusive b-hadrons OPAL Physics Note PN334/98.

[92] S. Blusk, Measurement of the Top Quark Mass, XXXIst Rencontres de Moriond, Les Arcs, France, 21-28 March 1998.

[93] D0 Collaboration, B. Abbott et al., Direct measurement of the top quark mass at D0, FERMILAB PUB-98/031-E submitted to Phys. Rev. D.

[94] The LEP collaborations, A Combination of Preliminary Electroweak Measurements and Constraints on the Standard Model, CERN-PPE/97-154.

[95] HRS Collaboration, C. R. Ng et al., ANL-HEP-PR-88-11 (1988).

[96] MAC Collaboration, H. R. Brand et al., Phys. Lett. B218 (1989) 369.

[97] TPC Collaboration, H. Aihara et al., Phys. Rev. D31 (1988) 2719.

[98] TPC Collaboration, H. Aihara et al., Zeit. Phys. C27 (1985) 39.

[99] HRS Collaboration, P. Baringer et al., Phys. Lett. B206 (1988) 551. 
[100] TPC Collaboration, H. Aihara et al., Phys. Rev. D34 (1986) 1945.

[101] JADE Collaboration, E. Elsen et al., Zeit. Phys. C46 (1990) 349.

[102] CELLO Collaboration, H. J. Behrend et al., Zeit. Phys. C47 (1990) 333.

[103] TASSO Collaboration, W. Braunschweig et al., Zeit. Phys. C48 (1990) 433.

[104] MARK J Collaboration, B. Adeva et al., Phys. Rep. C109 (1984) 131.

[105] JADE Collaboration, F. Ould-Saada et al., Zeit. Phys. C44 (1989) 567.

[106] TASSO Collaboration, W. Braunschweig et al., Zeit. Phys. C44 (1989) 365.

[107] AMY Collaboration, K. Ueno et al., Phys. Lett. B381 (1996) 365.

[108] VENUS Collaboration, M. Shirakata et al., Phys. Lett. B278 (1992) 499.

[109] TOPAZ Collaboration, E. Nakano et al., Phys. Lett. B340 (1994) 135.

[110] VENUS Collaboration, F. Hinode et al., Phys. Lett. B313 (1993) 245.

[111] VENUS Collaboration, K. Abe et al., Phys. Lett. B313 (1993) 288.

[112] V. Ruhlmann-Kleider, Search for Higgs bosons at LEP200, XXXIst Rencontres de Moriond, Les Arcs, France, 21-28 March 1998.

[113] T. Hambye and K. Riesselmann, SM Higgs mass bounds from theory, hep-ph/9708416.

[114] M. Peskin and T. Tacheuchi, Phys. Rev. D46 (1992) 381.

[115] G. Altarelli, R. Barbieri, and F. Caravaglios, Phys. Lett. B349 (1995) 145.

[116] M. Peskin, Beyond the Standard Model, lectures given at the 1996 European School of High Energy Physics, CERN 97-03, 49.

[117] M. Carena et al., Higgs Physics, CERN 96-01, Vol. 1, 351.

[118] J. Ellis, G. L. Fogli, and E. Lisi, Phys. Lett. B343 (1995) 282.

[119] T. Appelquist, J. Terning, and L. C. R. Wijewardhana, Phys. Rev. Lett. 79 (1997) 2767.

[120] F. Caravaglios and G. G. Ross, Phys. Lett. B346 (1995) 159.

[121] R. S. Chivukula, E. H. Simmons, and J. Terning, Phys. Rev. D53 (1996) 5258.

[122] M. Chiapetta et al., Z' Physics, CERN 96-01, Vol. 1, 577. 